NIST GCR 11-917-13

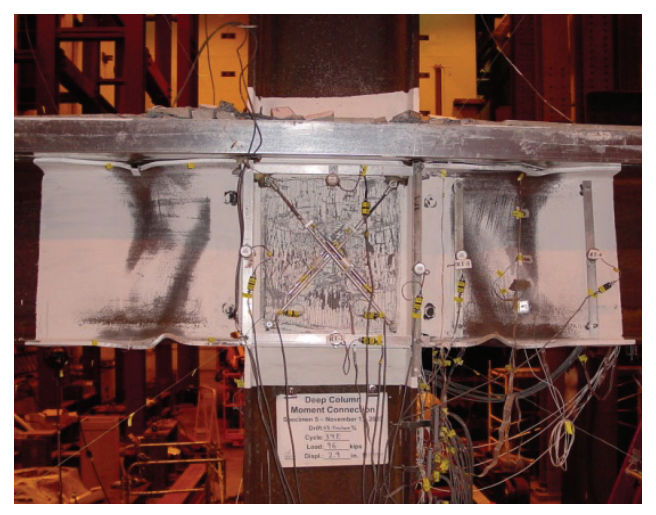

\title{
Research Plan for the Study of Seismic Behavior and Design of Deep, Slender Wide Flange Structural Steel Beam-Column Members
}

NEHRP Consultants Joint Venture

A partnership of the Applied Technology Council and the Consortium of Universities for Research in Earthquake Engineering
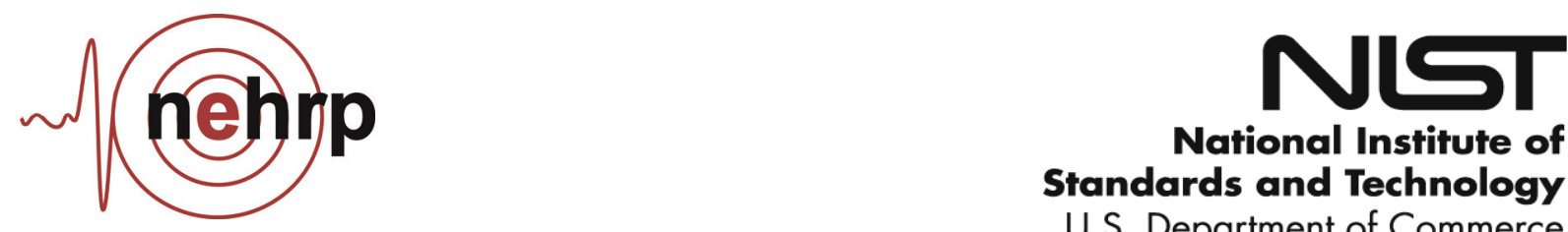


\section{Disclaimers}

This report was prepared for the Engineering Laboratory of the National Institute of Standards and Technology (NIST) under the National Earthquake Hazards Reduction Program (NEHRP) Earthquake Structural and Engineering Research Contract SB134107CQ0019, Task Order 10266. The contents of this publication do not necessarily reflect the views and policies of NIST or the U.S. Government.

This report was produced by the NEHRP Consultants Joint Venture, a joint venture of the Applied Technology Council (ATC) and the Consortium of Universities for Research in Earthquake Engineering (CUREE). While endeavoring to provide practical and accurate information, the NEHRP Consultants Joint Venture, the authors, and the reviewers assume no liability for, nor express or imply any warranty with regard to, the information contained herein. Users of information contained in this report assume all liability arising from such use.

Certain commercial software, equipment, instruments, or materials may have been used in the preparation of information contributing to this report. Identification in this report is not intended to imply recommendation or endorsement by NIST, nor is it intended to imply that such software, equipment, instruments, or materials are necessarily the best available for the purpose.

NIST policy is to use the International System of Units (metric units) in all its publications. In this report, however, information is presented in U.S. Customary Units (inch-pound), as this is the preferred system of units in the U.S. earthquake engineering industry.

Cover photo - Deep column moment connection test (courtesy of James Ricles, Lehigh University) 


\title{
Research Plan for the Study of Seismic Behavior and Design of Deep, Slender Wide Flange Structural Steel Beam-Column Members
}

\author{
Prepared for \\ U.S. Department of Commerce \\ National Institute of Standards and Technology \\ Engineering Laboratory \\ Gaithersburg, MD 20899
}

NEHRP Consultants Joint Venture A partnership of the Applied Technology Council and the Consortium of Universities for Research in Earthquake Engineering

December 2011

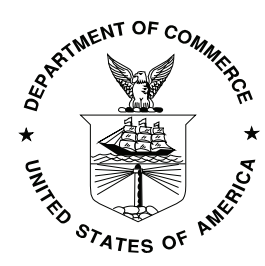

U.S. Department of Commerce John Bryson, Secretary

National Institute of Standards and Technology Patrick D. Gallagher, Under Secretary of Commerce for Standards and Technology and Director 



\title{
Participants
}

\author{
National Institute of Standards and Technology \\ John (Jack) R. Hayes, Jr., Director, National Earthquake Hazards Reduction Program \\ Steven L. McCabe, Deputy Director, National Earthquake Hazards Reduction Program \\ John (Jay) L. Harris III, Project Manager
}

\section{NEHRP Consultants Joint Venture}

Applied Technology Council

201 Redwood Shores Parkway, Suite 240

Redwood City, California 94065

www.ATCouncil.org

\section{Joint Venture Management Committee}

James R. Harris

Robert Reitherman

Christopher Rojahn

Andrew Whittaker

\section{Project Technical Committee}

James O. Malley (Project Director)

Charles J. Carter

Jerome F. Hajjar

Dimitrios Lignos

Charles Roeder

Mark Saunders
Consortium of Universities for

Research in Earthquake Engineering

1301 S. 46 ${ }^{\text {th }}$ Street, Building 420

Richmond, California 94804

www.CUREE.org

\section{Joint Venture Program Committee}

Jon A. Heintz (Program Manager)

Michael Constantinou

C.B. Crouse

James R. Harris

William T. Holmes

Jack Moehle

Andrew Whittaker

Project Manager

Ayse Hortacsu

\section{FEMA Representative}

Helmut Krawinkler 

The NEHRP Consultants Joint Venture is a partnership between the Applied Technology Council (ATC) and the Consortium of Universities for Research in Earthquake Engineering (CUREE). In 2007, the National Institute of Standards and Technology (NIST) awarded a National Earthquake Hazards Reduction Program (NEHRP) "Earthquake Structural and Engineering Research" contract (SB1341-07CQ-0019) to the NEHRP Consultants Joint Venture to conduct a variety of tasks, including Task Order 10266 entitled "Seismic Behavior and Design of Deep, Slender Wide Flange Structural Steel Beam-Column Members: Phase 1.”

The objective of this task was to develop a comprehensive long-range plan to research the seismic behavior of deep, slender wide flange structural steel beamcolumns in steel frames. Development of the plan included the investigation of available information on beam-column member and connection behavior in the literature, and considered the differences and interrelationships between member, subassemblage, and system performance. The resulting plan includes a summary of tasks for conducting both experimental and analytical research, and is intended to be the first step in the development of national consensus guidelines for designing and assessing the seismic performance of deep, slender wide-flange beam-columns in steel frame systems.

The NEHRP Consultants Joint Venture is indebted to the leadership of Jim Malley, Project Director, and to the members of the Project Technical Committee, consisting of Charlie Carter, Jerry Hajjar, Dimitrios Lignos, Charles Roeder, and Mark Saunders for their contributions in developing this report and the resulting recommendations. The NEHRP Consultants Joint Venture also gratefully acknowledges Jack Hayes (NEHRP Director), Steve McCabe (NEHRP Deputy Director), Jay Harris (NIST Project Manager), Mike Mahoney (FEMA), and Helmut Krawinkler (FEMA Technical Monitor) for their input and guidance in the preparation of this report, Ayse Hortacsu for ATC project management, and Peter N. Mork for ATC report production services.

Jon A. Heintz

Program Manager 



\section{Table of Contents}

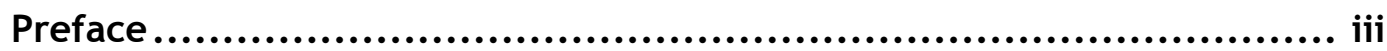

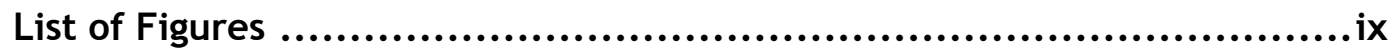

List of Tables.............................................................

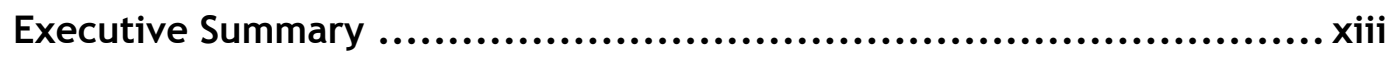

1. Introduction ...................................................... 1-1

1.1 Definition of Deep, Slender Beam-Column Members........................... 1-2

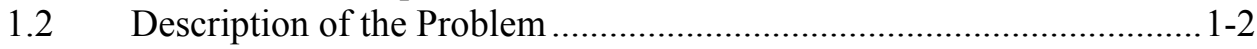

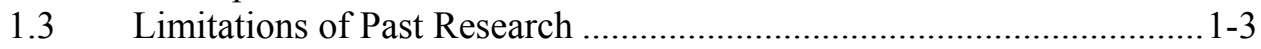

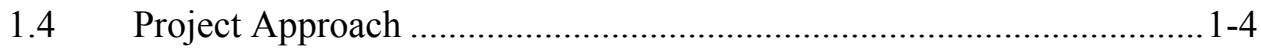

1.5 Report Organization and Content …................................................ 1-5

2. Key Issues Related to Performance of Deep, Slender Columns ...... 2-1

$2.1 \quad$ Common Failure Modes .......................................................................2-1

2.2 Key Issues Related to Performance ................................................ 2-2

2.3 Section and Member Slenderness .................................................2-2

2.4 Lateral Bracing and Lateral-Torsional Stability .................................2-3

2.5 Effect of Composite Slabs, Restraint, and Boundary Conditions ..........2-4

2.6 Strong-Column-Weak-Beam Ratio...................................................... 2-6

2.7 Effect of Connection Behavior on the Stability of Deep Columns ........2-7

2.8 Effect of Panel Zone Behavior on Deep Columns ...............................2-8

2.9 Column Base Connections ............................................................ 2-10

2.10 Range of Axial Load Ratios in Combination with Cyclic Loading .....2-11

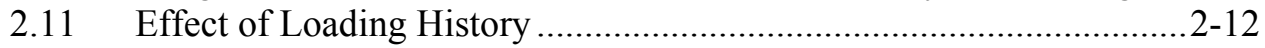

2.12 Modeling of Plastic Hinges in Deep Columns.................................. 2-12

3. Development of Archetype Buildings ............................... 3-1

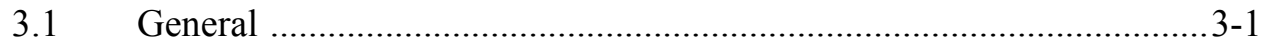

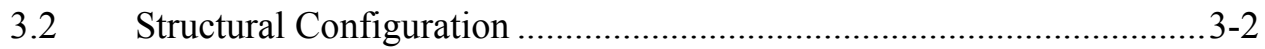

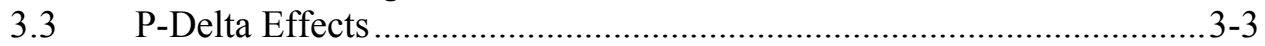

3.4 Level of Seismic Design Loading ......................................................... 3-3

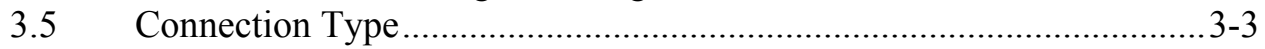

3.6 Strong-Column-Weak-Beam Ratio .................................................. 3-4

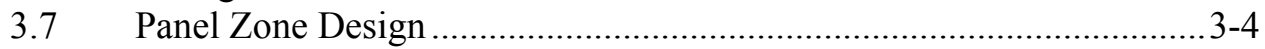

3.8 Column Base Connections .................................................................... 3-4

3.9 Design of Gravity Framing ...............................................................

4. Research Plan for Studying Member Behavior .......................4-1

4.1 Section Properties and Member Behavior .........................................4-1

4.2 Objectives for Member Investigations ............................................... 4-4 
4.2.1 Prior Research on Behavior of Deep Column Members .......... 4-5

4.3 Computational Research Plan .............................................................. 4-6

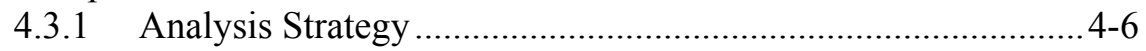

4.3.2 Member Selection..................................................................... 4-8

4.3.3 Boundary Conditions and Loading Protocols ......................... 4-11

4.3.4 Simulation of the Experimental Program ................................4-13

4.3.5 Assessment of Computational Research Results .....................4-13

$4.4 \quad$ Experimental Research Plan ........................................................ $4-13$

4.4.1 Experimental Strategy ...................................................... 4

4.4.2 Member Selection ................................................................ 4-14

4.4.3 Boundary Conditions and Loading Protocols .......................... 4-14

4.4.4 Assessment of Results ............................................................ 4-15

4.5 Corroborating Computational Research ..........................................4-15

4.6 Development of Improved Design Recommendations ........................4-16

5. Research Plan for Studying Subassemblage Behavior ................5-1

$5.1 \quad$ Objectives for Subassemblage Investigations......................................5-1

5.2 Computational Research Plan ......................................................5-2

5.2.1 Perform Continuum Nonlinear Analysis of Moment Frame Subassemblages .................................................................. 5-2

5.2.2 Establish the Accuracy and Reliability of Panel Zone Predictions ..........................................................................

5.2.3 Evaluate Fundamental Theories of Lateral Support and Lateral-Torsional Buckling......................................................5-5

5.3 Experimental Research Plan ................................................................5-5

5.3.1 Initial Testing on Deep Column Subassemblages ....................5-6

5.3.2 Investigation of Slab Effects, Lateral Support, and Selected Design Parameters................................................... 5-6

5.3.3 Investigation of Additional Issues ..................................... 5-7

5.4 Corroborating Computational Research ............................................. 5-8

5.5 Development of Practice-Oriented Analytical Tools and Improved Design Recommendations ............................................................. 5-9

6. Research Plan for Studying System Behavior..........................6-1

6.1 Objectives for System-Level Investigations .......................................6-1

6.2 Computational Research Plan ........................................................... 6-1

6.2.1 Preliminary Computational Studies .......................................... 6-2

6.2.2 Advanced Computational Studies.......................................... 6-5

6.3 Experimental Research Plan ...................................................... 6-6

6.3.1 Hybrid Simulation Tests .......................................................... 6-7

6.3.2 Earthquake Simulation Tests ............................................ 6-8

6.4 Corroborating Computational Research ........................................... 6-9

6.5 Development of Improved Design Recommendations ......................... 6-9

7. Research Plan: Summary .......................................... 7-1

7.1 Research Plan Objectives...................................................................

7.2 Research Plan Overview ............................................................... 7-1

7.3 Development of Archetype Buildings ............................................. 7-3

7.4 Investigation of Member Behavior ....................................................

7.5 Investigation of Subassemblage Behavior .......................................... 7-4

7.6 Investigation of System Behavior .................................................... 7-6 


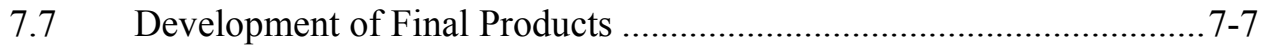

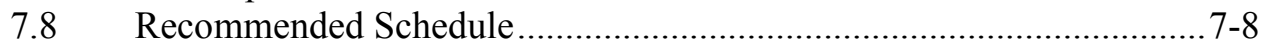

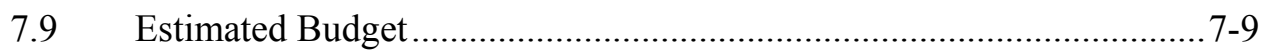

7.10 Key Collaborators ....................................................................

Appendix A: Literature Review .........................................A-1

A.1 Centrally Loaded Columns ........................................................... A-1

A.2. Research on Beam-Columns......................................................... A-3

A.2.1 Uniaxial Bending: In-Plane Strength ................................... A-3

A.2.2 Uniaxial Bending: Lateral-Torsional Buckling ...................... A-5

A.2.3 Biaxial Bending of Slender Beam-Columns ........................... A-5

A.2.4 Behavior of Beam-Columns under Reversed Cyclic

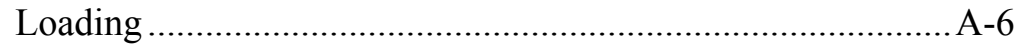

A.3. Research on Beam-to-Column Connections ......................................A-9

A.3.1 Boundary Conditions ...............................................................A-9

A.3.2 Column Behavior.......................................................... A-10

A.3.3 Panel Zone Behavior............................................................A-13

A.3.4 Strong-Column-Weak-Beam Design .................................A-16

A.3.5 Lateral Bracing of Beams and Columns ............................... A-17

A.3.6 Effect of Slab on Cyclic Response of Beam-to-Column Connections ...................................................................... A-19

A.3.7 Effect of Loading History on Seismic Performance of Beam-to-Column Connections ............................................. A-21

A.4 Research on Column-to-Foundation Connections ............................. A-22

A.4.1 Exposed Base Plates ............................................................A-23

A.4.2 Embedded Column Base Plates ............................................A-25

A.4.3 Research on Column Splices ................................................ A-26

A.5 Structural System Investigations.................................................A-27

A.5.1 Steel Building Simulation Models .......................................A-28

A.5.2 Structural System Performance ...........................................A-33

A.6 Common Failure Modes in Deep, Slender Wide Flange Structural Steel Beam-Column Members ............................................................. A-36

A.6.1 Global Failure Mechanisms .............................................A-36

A.6.2 Local Buckling of Plate Elements......................................A-38

A.7 Summary and Limitations of Design Criteria for Deep, Slender Wide-Flange Structural Steel Beam-Column Members ...................... A-41

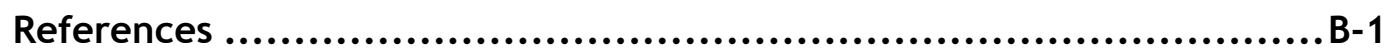

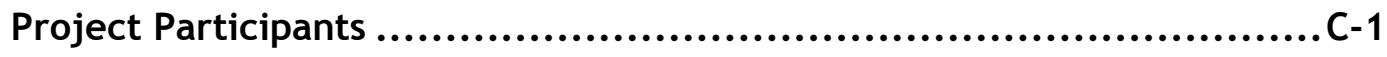



Figure 2-1 Cyclic response of a W27×194 column under axial load and bending

Figure 2-2 Effect of additional lateral bracing near reduced beam sections..... 2-5

Figure 2-3 Comparison of nominally identical test specimens with different boundary conditions .................................................................... 2-5

Figure 2-4 Inelastic deformation pattern of cruciform specimens with weak columns

Figure 2-5 Results from connection tests of RBS beams with deep columns:

(a) balanced panel zone design; (b) strong panel zone design ........ 2-8

Figure 2-6 Subassemblage total plastic rotation versus $V_{p z} / V_{p} \ldots \ldots \ldots \ldots \ldots \ldots \ldots \ldots . . . . . . . . .2-9$

Figure 2-7 Cyclic response of a W27 $\times 146$ column under combined axial load and bending.....

Figure 2-8 Effect of loading history on hysteretic response of nominally identical RBS beam-to-column connections................................. 2-12

Figure 2-9 Comparison of different component simulation models.............. 2-13

Figure 4-1 Variation in $r_{x} / r_{y}$ for wide flange sections ranging from W4 to W44.

Figure 4-2 Variation in warping constant, $C_{w}$, for wide flange sections ranging from $\mathrm{W} 4$ to $\mathrm{W} 44$......

Figure 4-3 Variation in torsional constant, $J$, for wide flange sections ranging from $\mathrm{W} 4$ to $\mathrm{W} 44$....

Figure 4-4 Variation in flange slenderness, $b_{f} / 2 t_{f}$, for wide flange sections ranging from $\mathrm{W} 4$ to $\mathrm{W} 44$

Figure 4-5 Variation in web slenderness, $h / t_{w}$, for wide flange sections ranging from $\mathrm{W} 4$ to $\mathrm{W} 44$...

Figure 5-1 Subassemblage configurations recommended for story-based research

Figure 6-1 Comparison between ASCE/SEI 41-06 and modeling options based on PEER/ATC-72-1

Figure 6-2 Calibration of the deduced moment rotation diagram of a composite beam with RBS. 
Figure 7-1 Approximate overall schedule for the recommended research

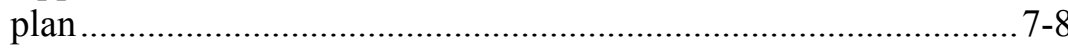

Figure 7-2 Approximate detailed schedule showing subtasks and interrelationships between major elements of the recommended research plan

Figure A-1 Hysteretic response of two fixed-ended heavy columns subjected to combined axial load and bending

Figure A-2 Cyclic response of a W27 $\times 146$ column under combined axial load and bending

Figure A-3 Typical joint panel zone behavior

Figure A-4 Results from connection tests of RBS beams with deep columns: (a) balanced panel zone design; (b) strong panel zone design

Figure A-5 Effect of additional lateral bracing near reduced beam sections

Figure A-6 Comparison of the hysteretic response of bare steel and composite beams in a perimeter frame.

Figure A-7 Comparison of the hysteretic response of bare steel and composite beams in an interior frame

Figure A-8 Effect of loading history on hysteretic response of nominally identical RBS beam-to-column connections

Figure A-9 Exposed and embedded column base plates

Figure A-10 Comparison of different component simulation models A-28

Figure A-11 Comparison between ASCE/SEI 41-06 and modeling options based on PEER/ATC-72-1

Figure A-12 Flexural buckling of a steel wide flange column A-37

Figure A-13 Torsional-flexural buckling of a steel wide flange column

Figure A-14 Buckling of steel columns with plastic hinges ........................... A-38

Figure A-15 Collapse due to dynamic instability 


\section{List of Tables}

Table 7-1 Research Plan - Summary of Tasks ......................................... 7-2

Table A-1 Summary of Capabilities of Hysteretic Models .......................... A-32 



\section{Executive Summary}

Current practice in modern steel frame design frequently concentrates seismic resistance into a few frames or a few bays within planar frames. In moment resisting frames, this practice results in column designs that are dominated by a combination of axial force and bending due to lateral deformation, rather than just axial force alone. Due to their increased in-plane flexural stiffness, deeper column sections are generally selected to satisfy drift criteria and to balance relative stiffness with deep beam sections. Columns that are generally stocky, in terms of both member stability and cross-sectional compactness requirements, are expected to perform well under seismic loading. Deeper, more slender columns, however, are more vulnerable to weak-axis and local buckling failure modes, and these sections must be checked against the potential for in-plane and out-of-plane instability under combined axial load and bending moment.

The term deep refers to member depths that are nominally greater than 16 inches (i.e., W16 sections and larger). The term slender refers to members that are sensitive to instability or have cross-sectional elements with width-to-thickness ratios approaching the seismic compactness limits for highly ductile members. An area of critical need identified by the American Institute of Steel Construction (AISC) is the seismic performance of deep, slender, wide flange structural steel beam-column members when subjected to a range of axial loads. Research on the stability of these members at large inelastic deformations is scarce, and a comprehensive research program is necessary to establish their behavior under seismic loading.

This report summarizes the recommended tasks, approximate schedule, and order of magnitude budget for a multi-phase, multi-year experimental and analytical research plan investigating the behavior of deep, slender wide-flange structural steel beamcolumn members. Work has been split up into three primary research components related to member, subassemblage, and system studies. The term member refers to an individual beam-column element, excluding connections to other elements in a building frame. The term subassemblage refers to a combination of beam and column elements representing a portion of the building frame. The term system generally refers to the overall building frame.

The objectives of this plan are: (1) the development of a comprehensive understanding of the seismic behavior of deep, slender wide flange beam-column members; (2) the ability to quantitatively simulate behavior and assess performance; 
and (3) the development of nationally accepted guidelines for the design and assessment of deep, slender wide flange beam-column members in seismic applications.

Based on the scope of the overall program, the estimated overall project schedule is approximately eight years. Due to complex interrelationships between the tasks and uncertainty in the procurement of contracted experimental research, this schedule should be considered approximate. Although the major research components can be considered separately, a significant level of interaction and coordination is required between many of the tasks to achieve the intended results.

Due to the competitive nature of the anticipated procurement process, potential variations in material and fabrication costs, and uncertainty in the current economic environment, establishing a precise estimate for each element of the research plan was not feasible. Based on historical expenditures for research on structural steel programs funded by the Federal government in the past, it is estimated that implementation of this program will require approximately $\$ 10 \mathrm{M}$, excluding systembased shake table testing, which has been identified as a Level 2 priority. An additional $\$ 2 \mathrm{M}$ is estimated to conduct the recommended shake table testing on fullscale, full-frame systems under the system-based research plan.

Although NIST will manage the overall program and perform selected portions of the work, the objectives of the research program would benefit from support, interaction, and coordination with other agencies of the Federal government, representative industry organizations, and codes and standards development organizations including:

- National Science Foundation (NSF)

- Federal Emergency Management Agency (FEMA)

- American Institute of Steel Construction (AISC)

- American Iron and Steel Institute (AISI)

- American Society of Civil Engineers (ASCE)

Opportunities to jointly fund research activities, or collaborate with these organizations, should be pursued. 


\section{Chapter 1}

Introduction

Current practice in modern steel frame design frequently concentrates seismic resistance into a few frames or a few bays within planar frames. In moment resisting frames, this practice results in column designs that are dominated by a combination of axial force and bending due to lateral deformation, rather than just axial force alone. Due to their increased in-plane flexural stiffness, deeper column sections are generally selected to satisfy drift limits contained in ASCE/SEI 7-10, Minimum Designs Loads for Buildings and Other Structures (ASCE, 2010), and to balance relative stiffness with deep beam sections. Columns that are generally stocky in terms of both member stability and cross-sectional compactness requirements of ANSI/AISC 341-10, Seismic Provisions for Structural Steel Buildings (AISC, 2010a), are expected to perform well under seismic loading. Deeper, more slender columns, however, are more vulnerable to weak-axis and local buckling failure modes. These sections, therefore, need to be checked against the potential for inplane and out-of-plane instability under combined axial load and bending moment as part of verifying member strength. However, published research on the stability of deep, slender beam-columns at large inelastic deformations associated with strong earthquake ground motions is limited, at best.

An area of critical need identified by the American Institute of Steel Construction (AISC) is the seismic performance of deep, slender, wide flange structural steel beam-column members when subjected to a range of axial loads. Though recent research on shallow, stocky columns (commonly used in braced frames) has suggested that beam-column members can maintain their structural integrity up to very high axial load ratios and large inelastic deformations, direct transference of these results to deep, slender beam-columns, which are more strongly influenced by differences in boundary conditions, remains a research need (see AISC, 2010a). A comprehensive research program is necessary to establish the behavior of deep, slender wide flange steel beam-column members subjected to seismic loading.

This report summarizes the scope of a recommended, comprehensive, long-range, experimental and analytical research plan to investigate the behavior of deep, slender wide flange structural steel beam-column members in seismic applications. The results from this research are intended to be used in the development of nationally accepted guidelines for the seismic design and assessment of deep, slender wide flange beam-column members. While emphasis is placed on special moment frame 
systems, application to intermediate moment frames, ordinary moment frames, and beam-column elements in braced frame systems, may also be possible.

\subsection{Definition of Deep, Slender Beam-Column Members}

For the purpose of this report, the term deep is defined as any column depth that is nominally greater than 16 inches (i.e., W16 sections and larger). The term slender is defined in relation to: (1) member sensitivity to instability; and (2) cross-sectional elements with width-to-thickness ratios approaching the seismic compactness limits for highly ductile members in ANSI/AISC 341-10. The term beam-column refers to members that are subjected to significant combined bending and axial loads as a result of seismic loading. In this report, the term column is used interchangeably with beam-column.

\subsection{Description of the Problem}

In moment resisting frames, accepted design practice assumes input energy during strong ground shaking is dissipated through plastic hinging in beams and, to some extent, through shear distortions in joint panel zones. To force plastic hinges to form in the beams, columns (i.e., beam-columns) must be designed with sufficient strength and stiffness to resist the maximum demands generated within the beams. This results in moment demands in the columns that are significantly larger than predicted by common analysis methodologies. Today, practitioners employ the effective length method (i.e., $K$ factor) to design beam-columns, with most applications of this method violating the design assumptions used in establishing the effective length. Inelastic stability at large deformations is a highly complex phenomenon, and current design recommendations promote simplified methods based on direct second-order inelastic analysis for design. However, the use of such methods is not directly applicable to seismic frames subjected to large inelastic deformations. Without experimental verification and calibration with advanced analytical models, this problem will remain difficult to solve.

A capacity design approach is provided by a strong-column-weak-beam (SCWB) criterion for special moment frames in ANSI/AISC 341-10. The SCWB criterion is

not required in less-ductile systems, i.e., intermediate and ordinary moment resisting frames. The intent of the SCWB criterion is to design columns to remain essentially elastic (except at the base), but it is one of the least understood seismic provisions in steel design (AISC, 2010a). Because individual frame columns cannot buckle independently of their story action in-plane, questions remain on how to apply storylevel buckling procedures at large deformations. Furthermore, the SCWB criterion aims to protect columns from section-level yielding, but it does not protect against member stability failure. Section properties of deep, slender wide flange sections make them more susceptible to stability failures; large differences have been 
observed between theoretical strength and actual strength considering local buckling failure modes.

Plastic hinges are expected to form in the columns at the base of moment resisting frames undergoing large deformations. To model this behavior, hysteretic response needs to adequately capture axial load-moment (P-M) interaction effects. Most uniaxial P-M interactions currently in use have been developed based on earlier research on small column sections, at low levels of axial force, and limited levels of ductility. There is a significant lack of information on the complete hysteretic behavior of larger wide flange sections as a function axial force, including strength and stiffness degradation.

\subsection{Limitations of Past Research}

Following the 1994 Northridge Earthquake, the Federal Emergency Management Agency (FEMA) funded a joint venture between the Structural Engineers Association of California (SEAOC), Applied Technology Council (ATC), and California Universities for Research in Earthquake Engineering (CUREE), known as the SAC Joint Venture, to address immediate and long-term needs for solving observed problems with welded steel moment resisting frame connections.

The SAC Joint Venture Project was a multi-phase, multi-year effort of experimental and analytical investigation on the performance of steel moment resisting frame connections of various configurations (FEMA, 2000a). It included extensive analysis of prototype buildings, detailed finite element and fracture mechanics investigations, material behavior studies, and full-scale testing of beam-to-column connection subassemblages. It concluded in 2000, with the publication of recommended design criteria for new steel moment frame buildings, evaluation and upgrade criteria for existing buildings, post-earthquake inspection and repair criteria, recommended specifications and quality assurance guidelines for steel moment frame construction, and a series of state of the art reports on steel moment frame buildings and connections.

Although the SAC Joint Venture Project had far-reaching implications on steel design and construction practice, the scope of the project was focused on the specific issue of moment resisting frame connections. While some of the resulting experimental and analytical information has been extrapolated to beam and column member performance, the behavior of deep, slender beam-column members was not a primary objective of this work.

Similarly, there has been a significant investment in research on steel member behavior and performance and considerable information available in the literature. A comprehensive summary of relevant information is provided in Appendix A, but much of this information is not specific to deep, slender, beam-column members. 
With little available information on column performance under the combination of high axial load and significant flexural ductility, experimental and analytical research that is directly applicable to deep, slender wide flange sections, and the boundary conditions inherent in moment resisting frame systems is needed.

\subsection{Project Approach}

In developing the recommended research plan, an extensive literature search of relevant research information was conducted. Based on the information collected, key issues related to the performance of deep, slender columns were identified. These issues were considered further and ultimately formed the basis of the recommended components of the analytical and experimental research program.

Each issue was categorized according to its relation to individual member, subassemblage, or overall system performance. In this report, the term member is defined as an individual beam-column element, excluding connections to other elements in a building frame. The term subassemblage refers to a combination of beam and column elements representing a portion of the building frame. Often a subassemblage will take the form of a cruciform consisting of a single column element connected to one or two beam elements, but it can also consist of multiple beams and columns representing a single line of framing or one story of a larger building frame. The term system generally applies to the overall building frame, but it can also refer to subsystems that include multiple framing lines or multiple stories of a building frame.

The program includes a major effort to establish prototype building configurations that will form the basis for the selection of members, subassemblages, and systems to be studied. These prototypes, designated archetype buildings, are intended to be representative of the range of structural configurations that would be expected to employ deep, slender wide flange columns.

In establishing the elements of the overall program, it was recognized that there is a significant interrelationship between the designations of member, subassemblage, and system described above. Integration of the three major elements of research is a critical aspect of the overall program. Although the elements of the recommended research plan have been highly structured and specifically identified, it is understood that the program will evolve as implementation occurs. Wherever possible during implementation, innovative ideas for new experimental and analytical approaches should be encouraged to resolve technical issues that cannot be addressed with present techniques or could not have been foreseen with present knowledge. 


\subsection{Report Organization and Content}

This report summarizes the scope of a recommended research plan to study the seismic behavior and design of deep, slender wide flange structural steel beamcolumn members. It outlines available information, defines key areas of need, provides recommendations for additional study and testing, and provides an order of magnitude estimate of the approximate level of effort. The recommended plan also considers appropriate coordination with other National Earthquake Hazard Reduction Program (NEHRP) partner agencies, representative industry organizations, and national model building codes and standards organizations. The remaining chapters of this report are organized as follows:

Chapter 2 identifies key issues related to the performance of deep, slender columns and provides a brief summary of relevant research.

Chapter 3 describes the development of archetype buildings.

Chapter 4 outlines recommendations for studying individual member behavior and performance.

Chapter 5 outlines recommendations for studying subassemblage behavior and performance.

Chapter 6 outlines recommendations for studying overall system behavior and performance.

Chapter 7 summarizes the recommended tasks and approximate schedule and budget for a multi-year program to conduct the research plan, and lists key collaborators that should be involved in such a program.

Appendix A provides a comprehensive summary of relevant research on steel beams, columns, connections, and systems. It includes a discussion on common failure modes observed in deep, slender, wide flange beam-column members, and a summary of limitations in presently available design criteria. 



\section{Chapter 2}

\section{Key Issues Related to Performance of Deep, Slender Columns}

This chapter provides a brief summary of relevant research and a description of key issues related to the performance of deep, slender wide flange columns in special moment resisting frames. Emphasis is placed on experimental and analytical investigation of the cyclic behavior of individual members, connection subassemblages, and overall moment frame systems. A comprehensive discussion of relevant research is provided in Appendix A.

\subsection{Common Failure Modes}

Critical failure modes observed in deep, slender wide flange structural steel beamcolumn members include:

Flexural buckling. Flexural buckling occurs when the axial load developed in a column attains the Euler load and the column buckles in a flexural mode.

Lateral-torsional buckling. Lateral-torsional buckling is characterized by lateral deflection and twisting of the cross-section when subjected to bending about the major principal axis.

Torsional or flexural-torsional buckling. To prevent buckling about the weak axis, wide flange columns are frequently restrained in the lateral direction. Flexuraltorsional buckling is defined by a rotation of the cross-section about the axis of lateral restraint. Depending on the location and configuration of the restraints, the column may react in a torsional deformation, or in a combined torsional and flexural deformation.

Local buckling. Local buckling of the cross-sectional elements can occur in several possible modes including: (1) buckling at the formation of plastic hinges; (2) pure local buckling involving out-of-plane deformation of the constituent plates; (3) distortional buckling involving in-plane bending of one or more of the constituent plates along with out-of-plane bending of all elements in a purely local mode; and (4) global buckling involving flexural or flexural-torsional buckling of plates with or without the interaction of local buckling.

More detailed information on failure modes of deep, slender wide flange sections is provided in Appendix A. 


\subsection{Key Issues Related to Performance}

The following issues were identified as critical for defining the basis of a recommended research plan investigating the behavior of deep, slender wide flange beam-column members:

- Section and member slenderness

- Lateral bracing and lateral-torsional stability

- Effect of composite slabs, restraint, and boundary conditions

- Strong-column-weak-beam ratio

- Effect of connection behavior on the stability of deep columns

- Effect of panel zone behavior on deep columns

- Column base connections

- Range of axial load ratios in combination with cyclic loading

- Effect of loading history

- Modeling of plastic hinges in deep columns

Many of the above issues are interrelated and will require consideration through a combination of member-, subassemblage- and system-based program elements.

Other issues are more specific to a single element of the recommended research plan.

\subsection{Section and Member Slenderness}

Differences in cross-section properties between shallow, stocky wide flange sections and deep, slender wide flange sections will affect column behavior and performance. The strong-axis dominance of slender sections makes them more susceptible to weak axis failure modes (e.g., flexural buckling, lateral torsional buckling), and higher width-to-thickness ratios can make them more susceptible to local failure modes (e.g., local buckling).

Newell and Uang (2006) analytically demonstrated that the cyclic behavior of deep (W27) columns subjected to high axial load is characterized by rapid strength deterioration due to severe flange and web local buckling. Figure 2-1 shows the response of a W27 $\times 146$ column subjected to a constant axial load of $0.55 P_{y}$ and reversed cyclic story drift ratios. In the figure, a rapid loss of strength occurs in the negative loading direction (i.e., compression). These results agree with findings from a recently developed database for deterioration modeling of steel components for slender wide flange sections (Lignos and Krawinkler, 2009). The pre-capping rotation capacity of these members ranges from about 1.5 to about 1.8 radians, and the post-capping rotation capacity (after first occurrence of local buckling) is not more than 0.15 radians. 

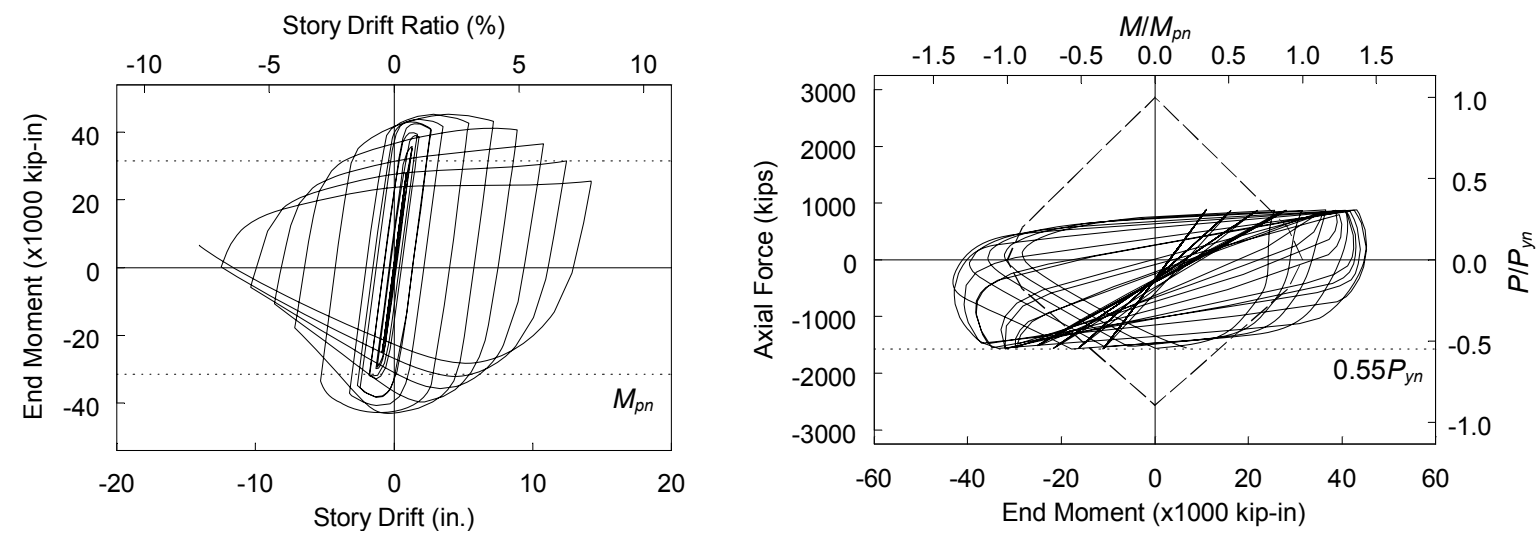

Figure 2-1 Cyclic response of a W27×194 column under axial load and bending (simulated results from Newell and Uang, 2006).

Because of the varying influences of different section properties, there will be a marked shift in behavior of deep, slender columns relative to stockier columns, and differences in the way that yielding and damage will progress through the crosssection.

\subsection{Lateral Bracing and Lateral-Torsional Stability}

ANSI/AISC 341-10 seismic design provisions require that lateral support for beams be applied at all plastic hinge locations, and that additional bracing be applied at regular intervals $\left(L_{b}\right)$ from these locations. Nakashima et al. (2002) conducted an analytical study of the lateral-torsional instability and lateral bracing effects of wide flange steel beams subjected to cyclic loading. It was found that AISC unbraced length requirements at the time were a reasonably conservative measure to ensure sufficient beam rotation capacity.

A comprehensive summary of investigations on lateral-torsional stability is provided in Trahair (1993). Most prior investigations are applicable to elastic buckling, but several have attempted to extend elastic buckling solutions to inelastic buckling. In practice, the form for elastic lateral-torsional buckling has been derived for constant bending of a doubly symmetric wide flange section under pure bending:

$$
M_{c r}=C_{b} \frac{\pi}{K_{y} L_{b}} \sqrt{\left(\frac{\pi E}{K_{z} L_{b}}\right)^{2} C_{w} I_{y}+G J E I_{y}}
$$

where $C_{w}$ is the warping constant, $J$ is the torsion constant, $E$ is the modulus of elasticity, $G$ is the shear modulus, $I_{y}$ is the moment of inertia about the minor y-y axis, $C_{b}$ is the lateral-torsional buckling modification factor for non-uniform moment diagrams when both ends of the segment, $L_{b}$, are braced, and $K_{y}$ and $K_{z}$ are the effective length factors for bending and torsion respectively (generally they are equal, expressed simply as $K$, and conservatively taken as unity). 
Equation 2-1 is adapted to mono-symmetric sections and other boundary and loading conditions through the use of factors (Clark and Hill, 1960). The equation shows that lateral-torsional buckling capacity has both warping and St. Venant (i.e., "pure") torsional components. Because $C_{w}$ is dependent upon the square of the distance between the centroid of the flanges, deeper wide flange sections generally have higher torsional resistance, but this occurs through increased resistance to warping torsion rather than St. Venant torsion.

Slender column sections may experience higher normal stresses due to warping restraint, and therefore earlier yielding of flanges (when combined with axial and bending stresses). The warping component of lateral-torsional buckling, however, is more severely reduced by a longer unsupported length, $L_{b}$. As a result, lateraltorsional buckling capacity of deep columns is expected to be much more strongly dependent upon lateral support than W12 or W14 columns, which are dominated by St. Venant torsion.

\subsection{Effect of Composite Slabs, Restraint, and Boundary Conditions}

When evaluating the behavior of beam-columns in moment resisting frames, it is usually necessary to consider the influence of the surrounding structure on the boundary conditions, which leads to the study of subassemblages and complete frames. Composite slabs are commonly used with beams supporting gravity loads, but are generally not considered in seismic design.

Detailed finite element simulations on various full-scale beam-to-column subassemblages by Kwasniewski et al. (1999) showed that axial restraint provided by the slab resulted in significant reduction in the post-buckling deterioration of steel beam-to-column connections. Concrete floor slabs in buildings are expected to provide this axial restraint, which is viewed as beneficial in reducing the adverse effects of lateral-torsional buckling. Assessment of the effect of composite slabs on the seismic performance of steel beam-to-column connections has been largely accomplished through the use of cruciform subassemblages. Due to beam boundary conditions in these subassemblages, however, it has been shown that the axial restraint provided by the slab is underestimated (Cordova and Deierlein, 2005).

A concrete slab that is integral with the beam and the column would be expected to restrain deformation and reduce the deterioration effects of twisting and out-of-plane movement of the beam and the column (Ricles et al., 2004). For composite steel beams with a gap between the slab and the column face, Tremblay et al. (1997) and Lignos et al. (2011e) showed that the bending strength of the beam in the two loading directions is nearly identical. The current design practice of separating the slab from the column face to limit composite interaction may limit the ability of the slab to impact the lateral-torsional stability of deep columns. 
Lignos and Krawinkler (2009) evaluated the effects of lateral bracing on seismic performance of more than 300 beam-to-column connections tested worldwide. It was concluded that additional lateral bracing was not effective in improving the precapping plastic rotation of steel beams, but that lateral bracing was effective in reducing the rate of cyclic deterioration, particularly for beams with reduced beam sections (RBS). This can be seen in Figure 2-2, which shows the deduced moment rotation diagrams of two nominally identical RBS connections with W30×99 steel beams, in which the insertion of additional lateral bracing close to the RBS region resulted in a small, but measurable improvement in hysteretic behavior.

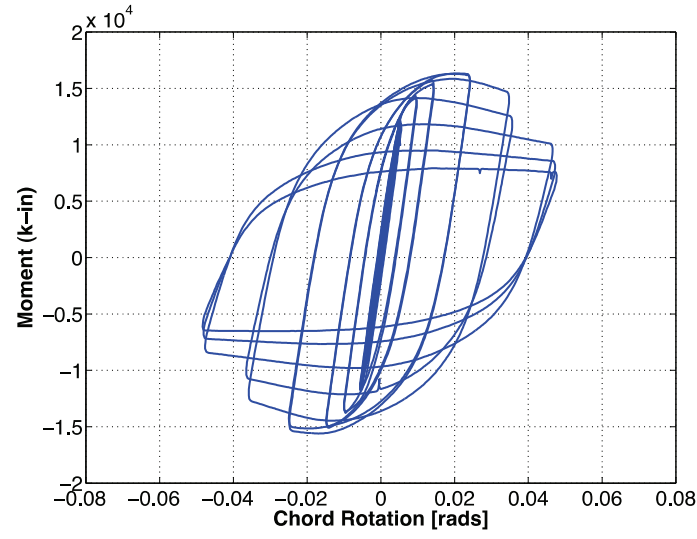

(a) Without additional bracing near the RBS

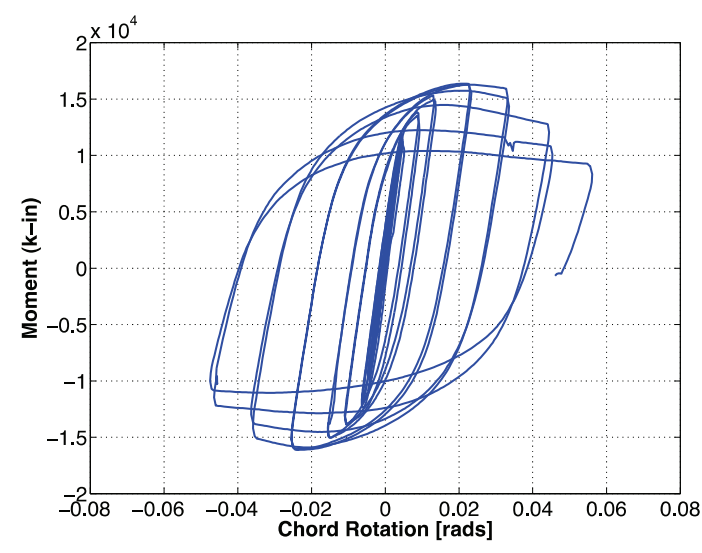

(b) With additional bracing near the RBS

Figure 2-2 Effect of additional lateral bracing near reduced beam sections (data from FEMA, 2000c).

Results from past experimental programs show that variation in test boundary conditions had a significant effect on system performance. This is illustrated in Figure 2-3.

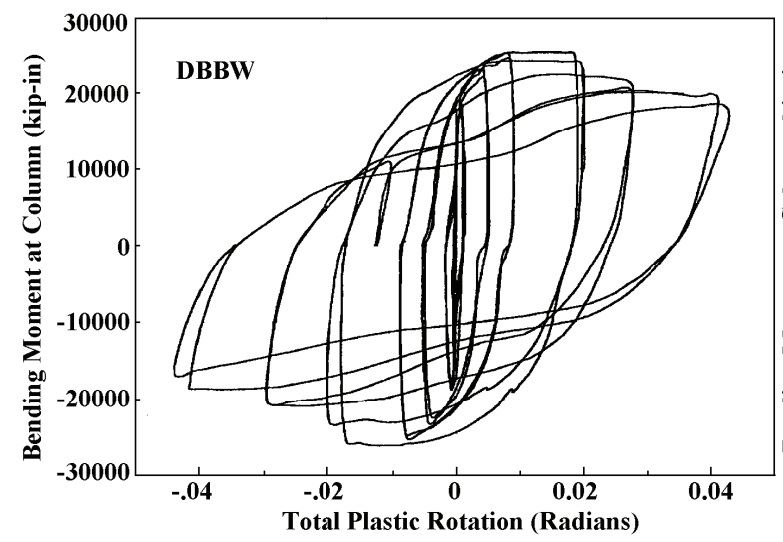

(a) DBBW - bolted web

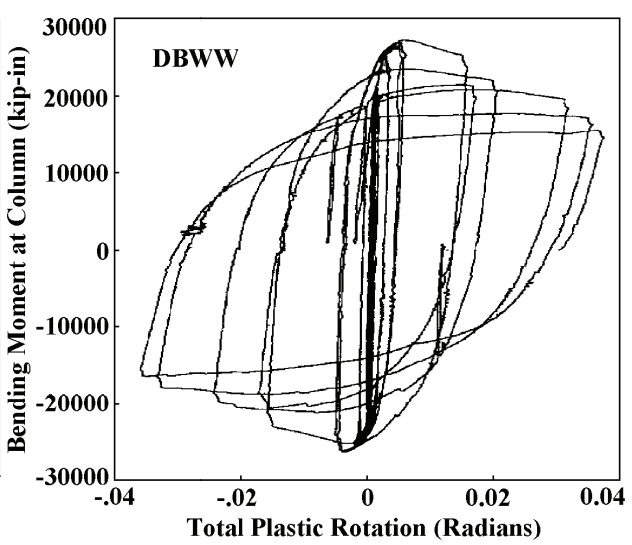

(b) DBWW - welded web

Figure 2-3 Comparison of nominally identical test specimens with different boundary conditions (FEMA, 2000c). 
In the figure, two nearly identical cruciform test specimens, DBBW (left) and DBWW (right), tested in different labs with different lateral support systems, exhibited unexpected differences in hysteretic behavior.

Appropriate characterization of the effects of the surrounding structure on performance is particularly important, because the effects of constraints and boundary conditions on all subassemblage tests must be well understood if the results are to be extrapolated for use in evaluating overall system behavior.

\subsection{Strong-Column-Weak-Beam Ratio}

Schneider et al. (1993) demonstrated that weak-column-strong-beam (WCSB) frames result in localized increases in seismic demands because inelastic deformations are concentrated in individual stories. Popov et al. (1975) conducted cyclic tests on interior cruciform beam-column subassemblages with axially loaded wide flange columns. Specimens were designed using a weak-column-strong-beam approach, and axial load ratios for different specimens were varied between approximately $30 \%$ and $80 \%$ of column yield. In columns subjected to larger axial loads, significant yield deformation and axial-shortening was noted and a C-shaped deformation pattern occurred after significant yielding (Figure 2-4). Plastic rotations developed unevenly in the top and bottom column segments, indicating the propensity for a story mechanism to form in systems with weak columns.

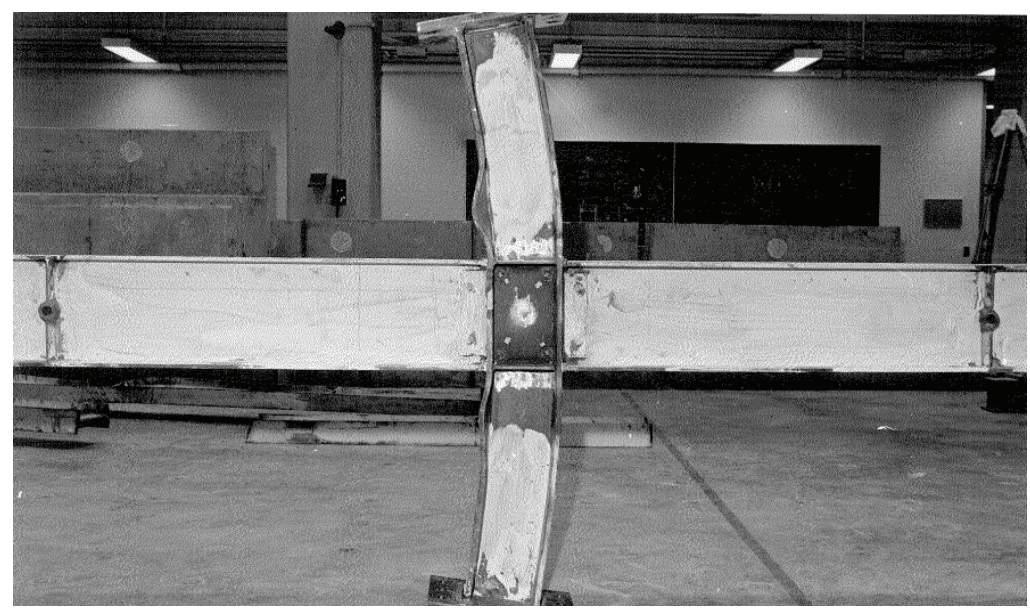

Figure 2-4 Inelastic deformation pattern of cruciform specimens with weak columns (Popov et al., 1975).

Nakashima and Sawaizumi (2000) suggested that column strength should be greater than $120 \%$ of the beam plastic moment capacity, $M_{p}$, to account for strain-hardening and to control the effects of concentration of plastic deformation. Recently, a fullscale collapse test of a four-story steel frame structure designed based on current Japanese seismic provisions was conducted at the E-Defense shaking table in Japan (Suita et al., 2008). The test structure collapsed in a first-story sidesway mechanism 
because of increased beam strength due to strain hardening, slab effects, and material variability. Columns were thin-walled tubes, and a strong-column-weak-beam ratio of 1.5 was employed. A high local slenderness ratio $(D / t=33)$, however, resulted in column strength deterioration due to local buckling and reduced flexural capacity. Numerical studies conducted by Lignos et al. (2011d) concluded that it would be possible to prevent the first story collapse mechanism of this structure if a strongcolumn-weak-beam (SCWB) ratio larger than 2.0 was used. This agrees with other analytical and experimental studies in the literature.

Current ANSI/AISC 341-10 seismic provisions attempt to control column yielding through the use of SCWB provisions that balance the probable plastic capacity of the beams with the nominal plastic capacity of the columns. Research has shown, however, that current SCWB ratios do not prevent column yielding because of: (1) strain hardening during inelastic deformation; (2) material variability; (3) composite effects; (4) variation in geometric corrections needed to transfer beam moment capacities to the column; and (5) varying locations of inflection points in beams and columns during seismic loading. Additional research investigating these effects on deep, slender column sections is needed.

\subsection{Effect of Connection Behavior on the Stability of Deep Columns}

Based on a few subassemblage experiments with deep columns, FEMA 355D State of the Art Report on Connection Performance (FEMA, 2000c) noted that the behavior of connections involving deep columns appears to be influenced by flange and web slenderness and by the stiffeners and doubler plates applied to the members. When used with reduced beam section connections, deep columns can also be subjected to twisting moments due to eccentric compression forces from the bottom flange of the beam as a result of lateral-torsional buckling.

Analytical studies by Shen et al. (2002) and analytical and experimental work by Ricles et al. (2004a) indicated that twisting of deep columns in RBS connections can be reduced with the presence of a floor slab and a supplemental brace located at the end of an RBS segment. The study concluded that the fracture potential and column twist in an RBS connection depends on the section modulus and torsional rigidity of the column section, where larger stresses in the column flange can lead to a potential for higher ductile fracture in the connection along with twisting of the column. If the stresses in the column flanges are lower, an RBS connection with a deeper column can have a smaller ductile fracture potential than an RBS connection with a shallower column.

Based on combined finite element and experimental studies, Ricles et al. (2004a) found that the ratio $h / t_{c f}^{3}$ or column depth, $d_{c}$, alone, are not clear indicators of the effects of an RBS connection on a deep column. Consideration must be given to the 
torsional and flexural properties of the column section and the size of the beam in the beam-to-column connection. Larger beams will result in a greater stresses applied to the column.

RBS connections to deep columns appear to perform better than welded unreinforced flange-welded web (WUF-W) connections to deep columns. Finite element parametric studies by Ricles et al. (2004b) show that WUF-W connections develop a larger plastic strains, resulting in greater potential for ductile fracture than RBS connections. Because of this, test results based on RBS connections to deep columns cannot be extrapolated to WUF-W connections to deep columns.

\subsection{Effect of Panel Zone Behavior on Deep Columns}

The effect of panel zone behavior on the seismic performance of steel moment resisting frames has been studied since the early 1970s. Krawinkler et al. (1971) showed that "controlled" inelastic behavior of panel zones combined with beam flexural yielding generally improves the overall seismic behavior of steel frames. This result has been confirmed in a number of analytical studies on steel moment frame systems (Krawinkler and Mohasseb, 1987; Gupta and Krawinkler, 1999). More recently, subassemblage tests on full scale composite RBS beam-to-column connections (Ricles et al., 2004; Lee et al., 2005) indicate that a balanced panel zone design (sharing of yielding between panel zones and beam plastic hinges) results in very little column twisting, no visible beam flange buckling, and almost no twisting of the RBS region compared to designs with strong panel zones (Figure 2-5).

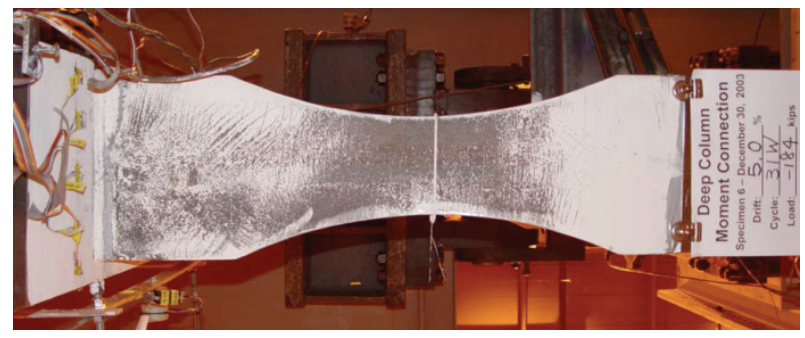

(a)

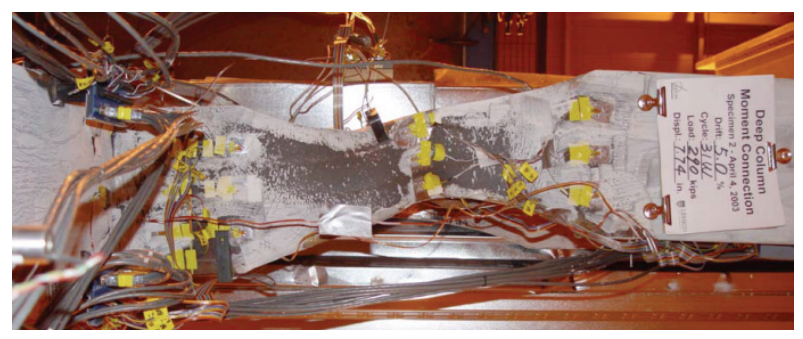

(b)

Figure 2-5 Results from connection tests of RBS beams with deep columns: (a) balanced panel zone design; (b) strong panel zone design (Ricles et al., 2004a).

While limited inelastic deformation in panel zones is beneficial to the behavior of steel moment resisting frames, excessive panel zone deformation has been shown to increase demands on the welds at beam-to-column connections, which can limit the inelastic deformation capacity of the connection. This effect is shown in Figure 2-6, in which the maximum inelastic rotation capacity achieved in moment resisting connections is compared to their relative panel zone resistance. $V_{p z}$ is the maximum measured panel zone shear force, and $V_{p}$ is the computed ultimate shear force capacity as proposed in Krawinkler and Mohasseb (1987), and currently approximated in AISC design provisions. The ultimate shear force capacity, $V_{p}$, 
includes capacity developed through significant strain hardening and inelastic deformation in the panel, so connections with $V_{p z} / V_{p}$ greater than 1.0 have significant panel zone shear distortions.

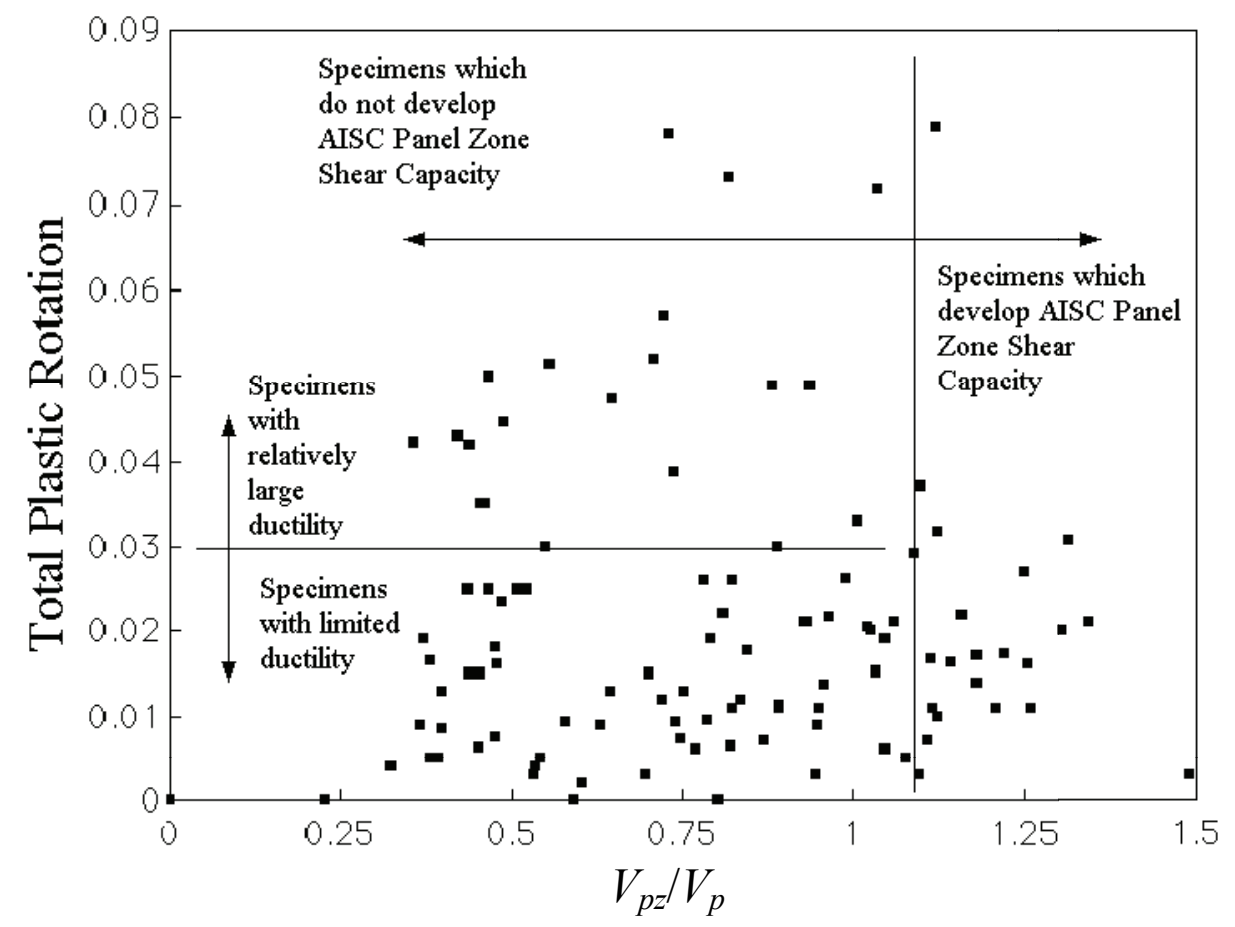

Figure 2-6 Subassemblage total plastic rotation versus $V_{p z} / V_{p}($ FEMA, 2000c).

The plot does not include the most recent test data, but it shows that specimens with clear panel zone yielding developed the largest plastic rotations, and that few specimens with extremely large panel zone yielding developed plastic rotation capacities consistent with current requirements for special steel moment resisting frame connections (i.e., 0.03 radians plastic rotation and 0.04 radians total rotation).

Tests reported in FEMA 355D support these observations. As a result, a balanced design procedure was recommended in which panel zone yielding is encouraged, but initiation of panel zone yielding is delayed until after initial flexural yielding of the beam in the connection. This concept has not been fully adopted into design provisions or common design practice. It can only be achieved in an approximate manner because a balanced condition should be based on the actual (and not the nominal) yield stress in the different steel sections, and because other considerations may result in a stronger panel zone than suggested by the equations. Moreover, sharing of inelastic deformation between beam plastic hinge deformations and panel zone shear deformations is a sensitive issue because of the relatively small post-yield stiffnesses of the components involved.

Numerical models for simulating panel zone behavior are available (Krawinkler, 1978; Kim and Engelhardt, 2002; Castro et al., 2005; Krishnan and Hall, 2006) but 
additional calibration with experimental data from subassemblage testing is necessary to improve the reliability of numerical prediction of moment frame behavior, and to investigate the concept of balanced panel zone design in more detail.

Deep columns may have greater interaction between shear yielding and shear buckling in the panel zone, but prior research does not show a clear indication that panel zone yielding will be of greater or lesser concern in frames with deep columns than frames with W12 or W14 columns. All available panel zone data, however, should be gathered and evaluated, and new data should be developed in order to improve upon presently employed design concepts and analytical models involving panel zone behavior as they are applied to deep, slender columns.

\subsection{Column Base Connections}

Column base connections can be categorized as exposed or embedded. Exposed column base plates are generally considered representative of a rotationally unrestrained (pinned) support condition and embedded column base plates are considered representative of a rotationally restrained (fixed) support condition. Summaries of column base connection studies are provided in Dewolf and Ricker (1990), Hensman and Nethercot (2000), Fahmy et al. (1999, 2000), Grauvilardell et al. (2005), and Gomez (2007).

Exposed column base plates in Grauvilardell et al. (2005) are categorized according to: (1) base plate behavior; (2) amount of restraint provided; (3) steel failure mode; (4) concrete failure mode; and (5) energy dissipation capacity. When exposed column bases are assumed to respond as fixed supports, they resist the applied loads only after large deformations that were neither modeled nor considered in the design. This results in larger than expected story drift ratios in steel frame structures, and sometimes structural collapse.

A thin column base plate can be characterized by ductile response since most of the inelastic behavior is concentrated in the base plate. A thick column base plate can be characterized by non-ductile response due to fracture of anchor rods, unless the rods have been designed to provide ductile behavior (Sato, 1987; Balut and Moldovan, 1997). In the case of weak-column-strong-connection details, a plastic hinge forms in the column. Depending on the weld metal toughness, however, it is possible for premature fracture of the column base welds to occur. In the case of strong-columnweak-connection details the potential for non-ductile behavior is recognized through a number of brittle failure mechanisms including crushing of the concrete and anchor rod fracture. Along with cracking of base plate welds, these failure mechanisms are classified as non-dissipative energy mechanisms.

Most column base research has focused on exposed column-base plate connections, but shallow or deeply embedded column-base plate connections have been common 
in both steel moment resisting frames and braced frames in regions of high seismicity. Embedded column base plates in Grauvilardell et al. (2005) are categorized in accordance with: (1) length of embedment; (2) column position in the frame (i.e., interior versus end column); (3) failure mechanism; and (4) deformation capacity.

Based on integrated experimental and analytical studies conducted by Pertold et al. (2000a, 2000b) wide flange columns are considered fixed when the embedment lengths range from $1 D$ to $2 D$, with $D$ being the lateral dimension of the column crosssection in the plane of bending. Full hysteretic loops are often observed for deeply embedded column base plates. The axial strength of the base plate connection is governed by two mechanisms: (1) for compression, the bearing of the end of the column on the bottom of the concrete foundation and (2) for compression or tension, the bond strength between the steel and concrete.

Analytical and experimental studies have demonstrated that most column bases exhibit some level of partial fixity, which significantly influences the boundary condition at the base of a column. Explicit investigation of the level of fixity and potential failure mechanisms of typical base connections, as applied to deep, slender columns, is needed.

\subsection{Range of Axial Load Ratios in Combination with Cyclic Loading}

Deep, slender columns in moment resisting frames will be subjected to combined axial load and lateral loads (bending). Axial loads will include tributary gravity loads and additional cyclic axial forces due to global overturning moments. Forces due to global overturning moments alternate between tension and compression, and are most significant in end columns of moment resisting frames. Finite element analysis results based on the data from Newell and Uang (2006) determined that the cyclic behavior of deep W27 columns exhibited significant strength degradation due to flange and web local bucking. In Figure 2-7, the simulated hysteretic behavior of a W27 $\times 146$ column shows the sensitivity of the response due to varying axial load.

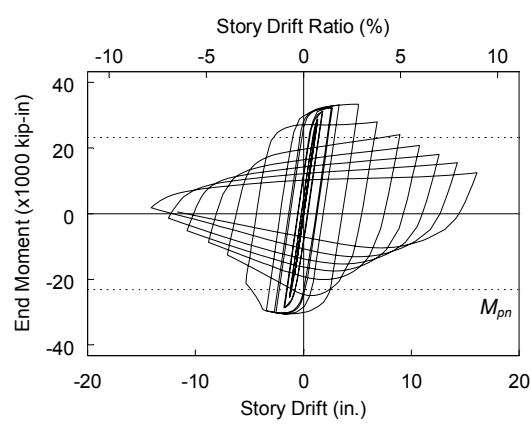

(a) $35 \% P_{y}$

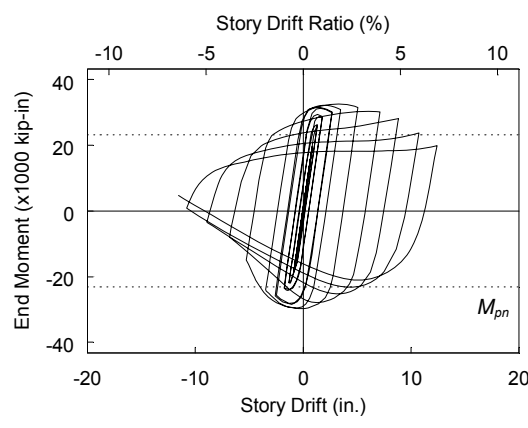

(b) $55 \% P_{y}$

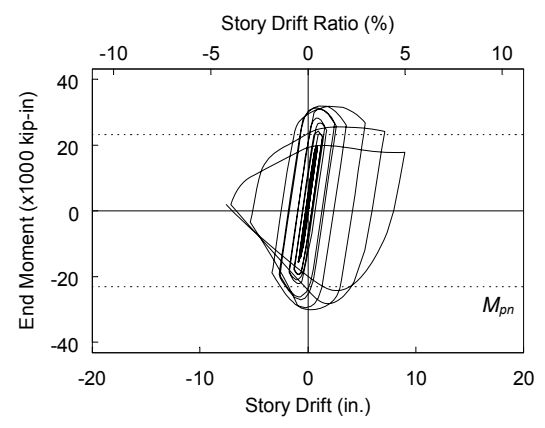

(c) $75 \% P_{y}$

Figure 2-7 Cyclic response of a W27 $\times 146$ column under combined axial load and bending (simulated results from Newell and Uang, 2006). 
Because of the observed influence of axial load on response, axial load ratio should be part of the loading protocol used to investigate the interaction of axial load and uniaxial/biaxial bending and drift on the behavior of deep, slender columns.

\subsection{Effect of Loading History}

Load and deformation history can have a significant impact on the performance of moment resisting connections (FEMA 355D; Clark et al., 1997; Lignos and Krawinkler, 2009; Krawinkler, 2010; Lignos and Krawinkler, 2011a). As part of FEMA 355D, Yu et al. (2000) tested full-scale, welded web, RBS connections to study the effect of loading history on performance. Two different loading protocols were used: (1) a symmetric loading protocol; and (2) a near-fault protocol that contained a pulse reversal in the loading history. Sample results from these tests are shown in Figure 2-8. The study concluded that, due to single high intensity pulsetype inputs, specimens tested with the near-fault protocol were able to reach $70 \%$ more plastic deformation capacity than specimens tested with the symmetric loading protocol.

Recent experimental and analytical studies related to collapse assessment of moment resisting frames (e.g., Lignos and Krawinkler, 2009; Lignos et al., 2011a) have demonstrated that component response depends on the performance level of interest. Components responding at or near collapse will have different response histories than components responding to design level events. Investigation of the effects of loading history on the response of deep, slender columns is needed.

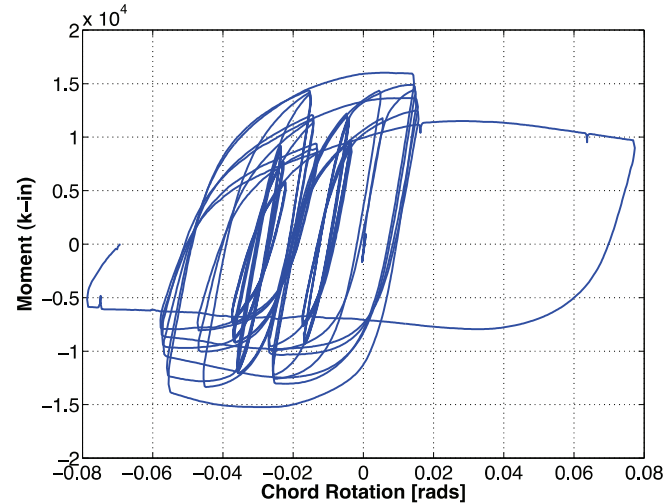

(a) Near-fault loading protocol

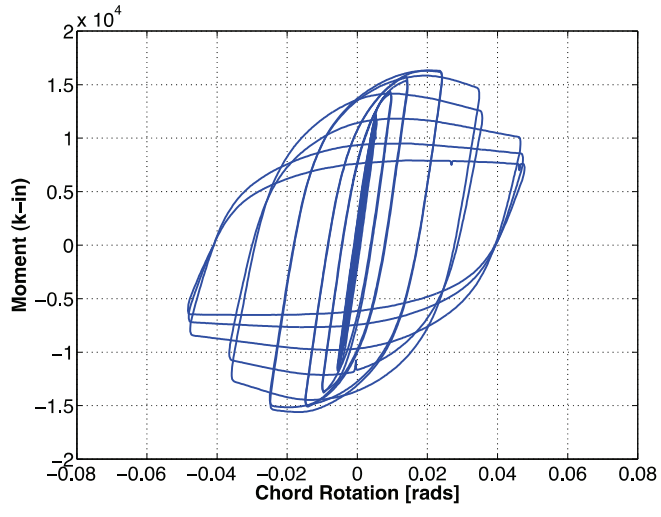

(b) Symmetric loading protocol

Figure 2-8 Effect of loading history on hysteretic response of nominally identical RBS beam-to-column connections (data from FEMA, 2000c).

\subsection{Modeling of Plastic Hinges in Deep Columns}

NEHRP Seismic Design Technical Brief No. 4, Nonlinear Structural Analysis for Seismic Design, A Guide for Practicing Engineers (NIST, 2011) summarizes approaches for modeling the behavior of steel moment frames for inelastic effects 
including flexural plastic hinging, shear yielding, connection failure, and member instabilities due to local or lateral-torsional buckling.

Inelastic structural component models can be classified by the way that inelastic behavior is distributed through the member cross-section and along its length. Figure 2-9 shows a comparison of five idealized model types for simulating the inelastic response of beams-columns.
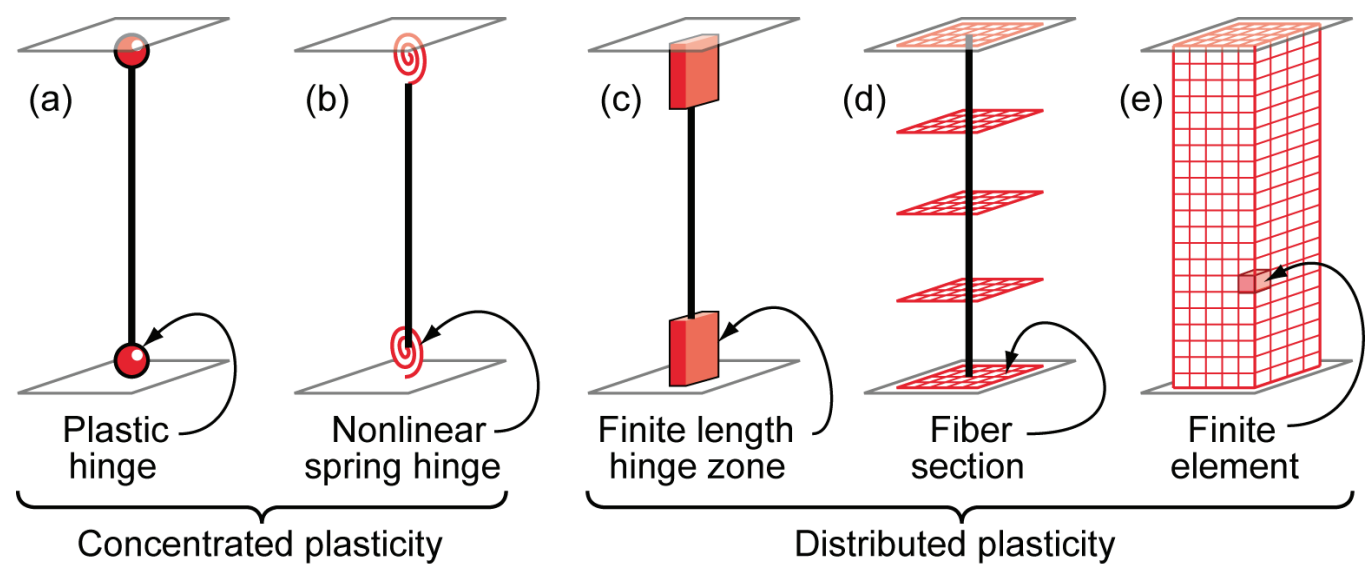

Figure 2-9 Comparison of different component simulation models (NIST, 2011).

The concentrated plasticity models shown in the figure are essentially zero length elements that simulate the moment-rotation relationship of a steel component. The distributed plasticity models shown in the figure include finite length hinge models with designated hinge zones at the member ends in which cross-sections are characterized through either nonlinear moment-curvature relationships or explicit fiber-section integrations based on the assumption that plane sections remain plane.

Modeling guidelines for steel components are summarized in PEER/ATC-72-1, Modeling and Acceptance Criteria for Seismic Design and Analysis of Tall Buildings (PEER/ATC, 2010). These guidelines are based on a database that was developed by Lignos and Krawinkler (2009) for modeling of the pre-capping plastic rotation, postcapping rotation and cyclic deterioration of beam sections with and without RBS. Krishnan (2010) developed an efficient fiber model that considers the interaction of axial load and bending, and simulates cyclic deterioration of steel columns due to local buckling, lateral-torsional buckling, and flexural buckling. However, calibration of the parameters that define nonlinear component models with experimental data on deep, slender columns is needed. 



\section{Chapter 3}

\section{Development of Archetype Buildings}

This chapter presents a plan for developing a set of archetype steel moment resisting frame buildings with deep, slender wide flange beam-column members. Emphasis is placed on special moment frame (SMF) systems. This set of archetypes will be used in both the analytical and experimental elements of the recommended research plan, and will be used to inform the range of parameters considered in the member, subassemblage, and system configurations that are studied as part of the plan.

\subsection{General}

Archetype buildings for special moment frame systems should be designed in accordance with the requirements contained within ASCE/SEI 7-10, Minimum Design Loads for Buildings and Other Structures (ASCE, 2010), ANSI/AISC 341-10, Seismic Provisions for Structural Steel Buildings (AISC, 2010a), ANSI/AISC 358-10, Prequalified Connections for Special and Intermediate Steel Moment Frames for Seismic Applications (AISC, 2010b), and ANSI/AISC 360-10, Specifications for Structural Steel Buildings (AISC, 2010c).

Development of archetype configurations should consider the guidance on archetype development contained in FEMA P-695, Quantification of Building Seismic Performance Factors (FEMA, 2009b). Prior to design, conclusions and findings from previous analytical studies on SMF systems should also be considered, including FEMA 355C, State of the Art Report on Systems Performance of Moment Steel Frame Buildings in Earthquakes (FEMA, 2000b) and NIST GCR 10-917-8, Evaluation of the FEMA P-695 Methodology for Quantification of Building Seismic Performance Factors (NIST, 2010).

The set of archetype buildings should encompass a range of design variables that address key issues related to the behavior of deep, slender columns discussed in Chapter 2. The following design variables should be considered:

- Structural configuration (in terms of number of bays, bay width, number of stories, and story height)

- P-Delta effects (in terms of tributary floor area)

- Level of seismic design loading (in terms of seismic design category) 
- Connection type (per ANSI/AISC 358-10)

- Strong-column-weak-beam (SCWB) ratio

- Panel zone design (balanced versus strong design)

- Column base connections

- Gravity framing

Design variables that should be considered in the development of a set archetype buildings using deep, slender wide flange beam-column members are discussed in the sections that follow.

\subsection{Structural Configuration}

Typical configuration variables (in terms of number of bays, $b$, bay width, $w$, number of stories, $N$, and story height, $h$ ) can affect the behavior of a seismic-force-resisting system. Variation in these design variables can influence global structural response parameters such as building period, base shear, lateral drift, and global overturning, as well as local member demands such as shear and flexural forces, axial load ratio, and the formation of plastic hinges.

Plan configuration. Use of deep, slender column members is generally associated with the concentration of moment resisting elements into a few frames or a few bays within planar frames. For this reason, the typical plan configuration should consist of perimeter steel moment resisting frames designed using deep column sections.

Configurations with distributed moment resisting frames are not likely to require the increased flexural strength and stiffness associated with deep column sections, and need not be considered.

Number of bays and bay width. The number of bays, $b$, and bay width, $w$, will affect the range of axial load ratios in the end columns of steel moment resisting frames (due to global overturning effects). The number of bays in archetypical frames should range from 2 to 8 . In recent past studies (FEMA, 2009b; NIST, 2010) typical bay widths of 20 feet and 30 feet have been used.

Number of stories and story height. Tall steel buildings are often designed using steel braced frames as the primary lateral resisting system or in conjunction with steel moment frames as part of a dual system. Pure moment resisting frames are most commonly used in buildings of about 20 stories or less. For this reason, it is recommended that the archetype buildings range in height from $N=1$ to 20 stories. In recent past studies (FEMA, 2009b; NIST, 2010) typical story heights of 13 feet in the first story and 12 feet in the other stories have been used. 


\subsection{P-Delta Effects}

Explicit consideration of P-Delta effects is required in each story where the elastic stability coefficient, $\theta$, exceeds 0.1 (ASCE/SEI 7-05, Equation 12.8.7). Because of the flexibility of steel moment resisting frame systems, P-Delta effects can control member sizes.

Variation in the tributary floor area will affect P-Delta considerations because different portions of the total axial load will be distributed to the columns. A range of tributary areas should be considered in the archetype building designs.

\subsection{Level of Seismic Design Loading}

Structural design requirements are keyed to Seismic Design Categories, which are classifications assigned to structures based on their occupancy risk category and the severity of earthquake ground motions expected at the site. In general, special moment frame structures are required in Seismic Design Categories D, E, and F, which include standard occupancy structures in regions of high seismicity, and essential facility structures in regions of moderate and high seismicity. The level of seismic design loading considered in the development of archetype buildings should be based on short-period and 1-second period spectral accelerations $\left(S_{s}\right.$ and $S_{l}$, respectively) that are consistent with values for moderate and high seismicity in Seismic Design Categories D and higher.

Additionally, design locations will dictate the selection and scaling of appropriate sets of ground motion records that represent the seismic hazard at the selected location. Ground motion selection should also address the effect of loading history on the behavior of steel moment resisting frames (e.g., ordinary far field, long duration, or near fault pulse ground motions). A set of far field, near fault, and long duration ground motions at a range of locations and intensities should be considered.

\subsection{Connection Type}

Past connection tests have shown that some connection types are inherently more stable than others (FEMA, 2000c; Lignos and Krawinkler, 2011). Variation in connection behavior has been shown to affect the behavior of deep, slender columns, but the number of tests that have been conducted has been limited.

Evaluation of the behavior of deep, slender columns in steel moment resisting frames will require explicit consideration of alternative prequalified beam-to-column connections as a design variable. Based on connections included in ANSI/AISC 358-10 and common U.S. practice, the following connection types should be considered in the development of archetype buildings:

- beams with reduced beam section (RBS), 
- welded unreinforced flange connection (WUF-W),

- bolted flange plate (BFP) connection, and

- extended end plate (EEP) connection.

\subsection{Strong-Column-Weak-Beam Ratio}

Strong-column-weak-beam (SCWB) ratios affect the distribution of yielding in steel moment frames. Design of archetype buildings should consider variation in SCWB ratios to investigate the effects of: (1) beam strain hardening during inelastic deformation; (2) material strength variability; (3) composite effects; and (4) varying location of inflection points in beams and columns during an earthquake. Changes in axial load due to variations in the tributary floor area recommended in Section 3.3 will also affect the SCWB behavior of archetype buildings. Based past experimental and analytical studies, the following SCWB ratios should be considered:

- $\mathrm{SCWB} \geq 1.0$ (design equation)

- $\quad \mathrm{SCWB} \geq 1.5$

- $\quad \mathrm{SCWB} \geq 2.0$

\subsection{Panel Zone Design}

While limited inelastic deformation in panel zones can be beneficial to the behavior of steel moment resisting frames, excessive panel zone deformation has been shown to limit the inelastic deformation capacity of connections. Based on connection types considered in Section 3.5, balanced panel zone design (matching of strength between the panel zone and beam) is recommended. It is recognized that panel zone strength may be a difficult parameter to control and that balanced design may not be feasible because of limitations in available column web thicknesses. Also, material strength variability should be considered.

\subsection{Column Base Connections}

Column bases are often a source of significant inelastic response in steel moment resisting frames, so explicit consideration of column base conditions is necessary. A distinction between exposed base plate connections and embedded connections should be made. Limit states including bearing capacity of the concrete foundation and the supporting soils, and the degree of rotational fixity provided by different base connections should be considered in archetype building designs.

\subsection{Design of Gravity Framing}

Past studies involving nonlinear response history analyses of moment resisting frames have shown that gravity framing affects the distribution of yielding within moment resisting frames and could help mitigate the potential for collapse due to 
lateral dynamic instability (FEMA, 2009a). Archetype buildings should consider typical gravity framing systems that are likely to be present. Gravity framing (e.g., beams, columns, and beam-to-column connections) should be designed to have sufficient strength and deformation capacity to resist tributary gravity loads at maximum story drift ratios, and archetype buildings should be explicitly designed considering the presence of the gravity system. 



\section{Chapter 4 \\ Research Plan for Studying Member Behavior}

This chapter describes a recommended plan for studying the behavior of deep, slender wide flange beam-columns as individual members. It outlines program objectives and necessary computational and experimental strategies for investigating member test specimens as part of the eventual development of improved member design provisions.

\subsection{Section Properties and Member Behavior}

Deep sections are often used in cases where large bending moments are anticipated about the major axis and additional stiffness is needed to limit drift in a structure. Axial and flexural strength about the minor axis are often assumed to be less critical for these members. Figure 4-1 plots the variation of the ratio $r_{x} / r_{y}$ for W4 to W14 sections and deep wide flange sections (W16 and larger). The average ratio for W4 to $\mathrm{W} 14$ sections is 2.4 , while the average ratio for $\mathrm{W} 16$ to $\mathrm{W} 44$ sections is 4.3 . Differences in $r_{x} / r_{y}$ are pronounced, and show the strong-axis dominance of the deeper sections. As a result, they will be more susceptible to weak-axis failure modes, especially those dependent on $I_{y}$, such as lateral-torsional buckling, flexural buckling and (less clearly) flexural-torsional or torsional buckling.

Similarly, the influence of torsion due to restraint of warping is a potential concern in deeper sections. Figure 4-2 plots the variation in warping constant, $C_{w}$, for W4 to W14 sections and deep wide flange sections (W16 and larger). The average value of $C_{w}$ for W4 to W14 sections is 7,730 in ${ }^{6}$, while the average value for W16 to W44 sections is $46,000 \mathrm{in}^{6}$. The larger warping constant in deep sections generally increases the torsional resistance and thus increases resistance to lateral-torsional buckling and flexural-torsional buckling. However, this is accomplished through enhanced resistance to warping of the cross-section. This could have secondary effects not seen in stockier sections that rely more on resistance due to St. Venant torsion. These include a build-up of additional normal stresses in the flanges that, when combined with stresses due to flexure about the major axis, may lead to earlier yielding in the flange tips due to restraint of warping. There will be a marked shift in behavior of deep sections relative to stockier columns, and the way in which damage progresses will be different because of the different dominant influences of the two types of torsion. 


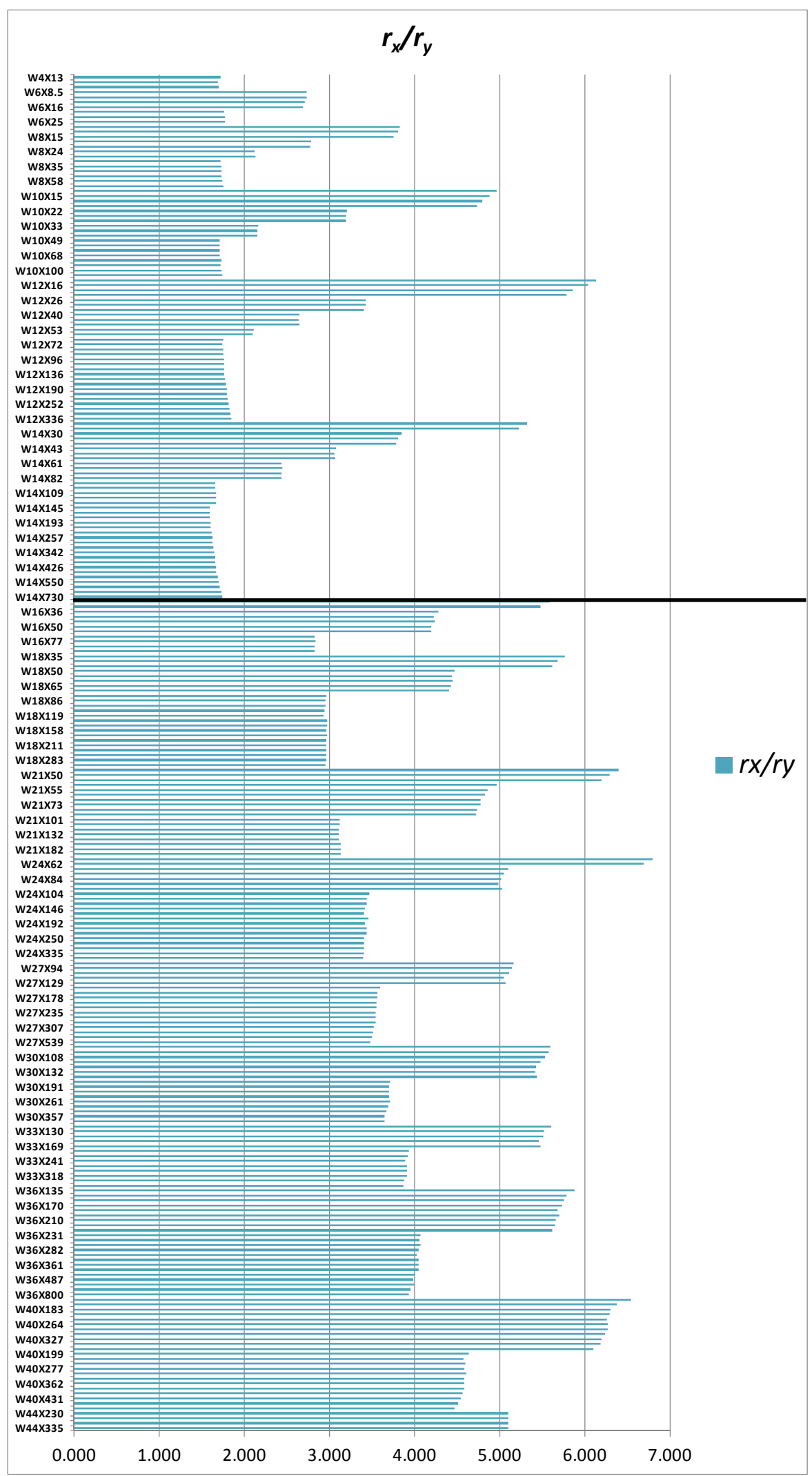

Figure 4-1 Variation in $r_{x} / r_{y}$ for wide flange sections ranging from W4 to W44. 


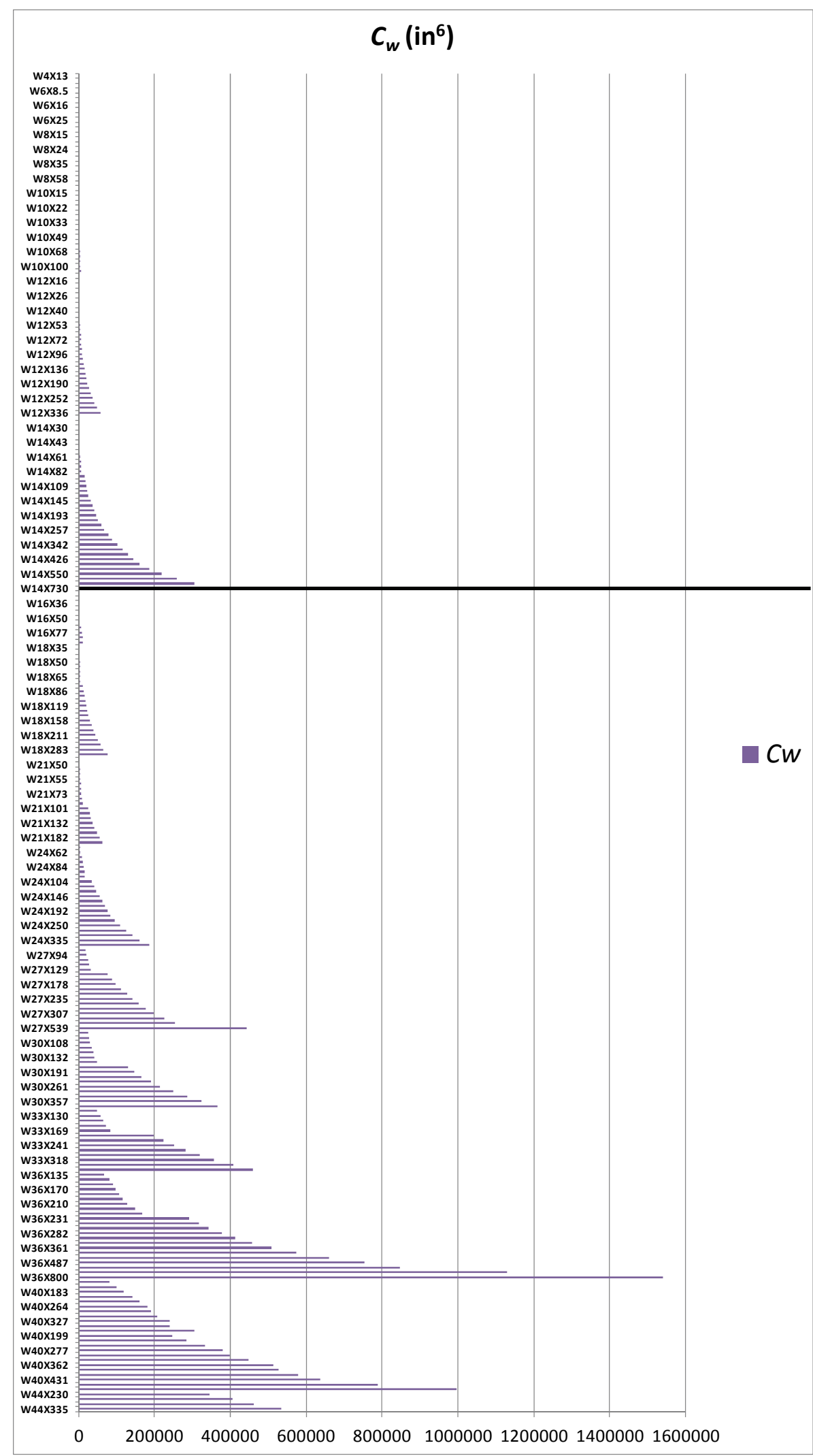

Figure 4-2 Variation in warping constant, $C_{w}$, for wide flange sections ranging from W4 to W44. 
In addition, while the axial load-moment interaction equation (Equation H1-1) that is used within ANSI/AISC 360-10 is valid for all structural members, it was calibrated largely to capture the response of wide flange members subjected to strong-axis flexure (Hajjar et al., 1997). The axial load-moment interaction behavior of deep columns subjected to strong-axis flexure should, therefore, be represented reasonably well by this equation. However, members subjected to weak-axis flexure may have significantly more flexural strength than represented by Equation H1-1. Equation H1-2, introduced in 2005, provided additional strength for compact members that are susceptible to out-of-plane flexural buckling, lateral-torsional buckling, or flexuraltorsional buckling failures. The use of Equation H1-1 to represent the section strength (i.e., plastic strength) of deep sections has not been thoroughly researched.

The fundamental response of columns in ANSI/AISC 341-10 is predicated on the use of moderately ductile or highly ductile members. Local buckling limits are provided to ensure that significant member yielding can occur prior to local buckling of the flange, web, or a combination of both. There are many wide flange sections W16 and larger that satisfy the compactness limits for moderately ductile and highly ductile members and can be used as deep beam-column members.

While the use of highly ductile members in special moment frames is emphasized, the behavior of a broader range of deep columns will be probed at the limits for both moderately ductile and highly ductile members to ensure that these limits provide a predictable progression of damage that adequately retains strength and stiffness during seismic loading. Additionally, the axial load-moment interaction behavior of deep columns in both strong-axis and weak-axis bending will be investigated to verify stable behavior under cyclic loading.

\subsection{Objectives for Member Investigations}

Objectives for investigating the behavior of deep, slender beam-column members include the following:

- Identification of failure modes and progression of damage in deep beam-column members. Deep sections are used primarily for strong-axis flexure combined with axial compression, but weak-axis flexure and axial tension may also be induced in some framing configurations and should be similarly explored.

- Determination of the sensitivity of deep beam-column members to boundary conditions, particularly in relation to out-of-plane stability failures (e.g., lateraltorsional buckling) after cyclic excursions into the inelastic range.

- Assessment of the accuracy of current AISC formulas for predicting the capacity associated with all deep beam-column limit state behaviors, and capacity design, lateral bracing, and other relevant provisions. 
Expected modes of failure in deep beam-column members include:

- cross-section plastification leading to plastic hinging in strong-axis or weak-axis flexure,

- significant yielding in axial compression,

- strong- and weak-axis flexural buckling, torsional buckling, flexural-torsional buckling, lateral-torsional buckling,

- flange local buckling, web local buckling, combined flange and web local buckling along with distortional buckling, and

- various combinations of the above local and global member failure modes.

It is expected that subassemblage and system studies will investigate member failure within the context of system behavior, including twisting that can occur at connections under stress, formation of story-level mechanisms, and system dynamic instability or collapse. Key questions to be addressed regarding member behavior include:

- Are the AISC formulas for weak-axis flexural buckling, lateral-torsional buckling, flexural-torsional buckling, and local buckling sufficiently accurate for deep columns? Many of these formulas were investigated predominantly for stockier cross-sections and monotonic loading. Assessing lateral-torsional buckling response can include determination of the accuracy of the bracing provisions in ANSI/AISC 341-10.

- How sensitive are these members to boundary conditions, particularly torsional buckling conditions? Are torsional failure modes with different boundary conditions being accounted for appropriately?

- How sensitive are these members to imperfections, particularly member sweep about the weak axis? Are these imperfections adequately accounted for in design, particularly in the presence of cyclic loading?

- Will distortional buckling modes influence the behavior in ways that are currently not accounted for in design? How do boundary conditions near connections affect distortional buckling modes?

- Does cyclic loading result in increased degradation of strength in deep beamcolumn members as compared to stockier columns? If so, under what conditions?

\subsubsection{Prior Research on Behavior of Deep Column Members}

While pure flexure in deep girders has been studied in prior research, the majority of work on columns (i.e., members in which the axial demand is more than $10 \%$ of the design axial strength) has focused on sections with nominal member sizes of W14 
and less, particularly for research that includes cyclic loading. Investigation of flexure in girders indicates that the members are sensitive to lateral-torsional buckling (particularly those with large $r_{x} / r_{y}$ values) as well as combined local and lateral buckling and distortional buckling within the cross-section (Bradford, 1992).

Newell and Uang (2006) conducted experiments on W14 sections subjected to axial compression plus biaxial flexure. Tested sections complied with ANSI/AISC 341-05 seismic compactness requirements, although the lightest section had a flange slenderness that was just at the limit of local buckling, and flange local buckling was observed in the cyclic response. This work was followed with a computational study that included W27 sections, which indicated that more severe strength degradation can occur in these members due to combined flange and web local buckling.

\subsection{Computational Research Plan}

System analyses conducted during the development of archetype buildings will have established representative sizes for deep beam-column members based on prototypical structural configurations, and will have identified baseline behavior of these members as part of these systems. Representative member sizes from archetype designs will provide a guide for selection of sizes for computational and experimental investigation of deep beam-column members.

A preliminary computational study should sweep through a set of boundary conditions and material properties that are sufficient to explore the range of behavior of deep beam-column members. This will help establish specific types of damage or behavior that should be investigated in more detail experimentally, and should identify critical issues for which current AISC provisions may not be sufficient. Through this study it will be possible to choose appropriate member sizes for experimental testing that represent the likely limits of behavior expected in these members.

\subsubsection{Analysis Strategy}

System-level analyses are being conducted under a separate element in the overall research plan. Analyses of member behavior should, therefore, concentrate on highfidelity simulations of deep beam-column behaviors. These analyses should include both geometric and material nonlinearity, and the member should be discretized adequately to capture all relevant nonlinear cyclic behavior in three dimensions.

Figure 4-3 plots the variation in torsional constant, $J$, for wide flange sections ranging from W4 to W44. Although the median value of $21 \mathrm{in}^{4}$ for deep column sections is larger than the value of $2.1 \mathrm{in}^{4}$ for standard column sections, St. Venant torsion combined with distortional buckling may make members with small torsional constants more susceptible to complex torsional failure modes. 


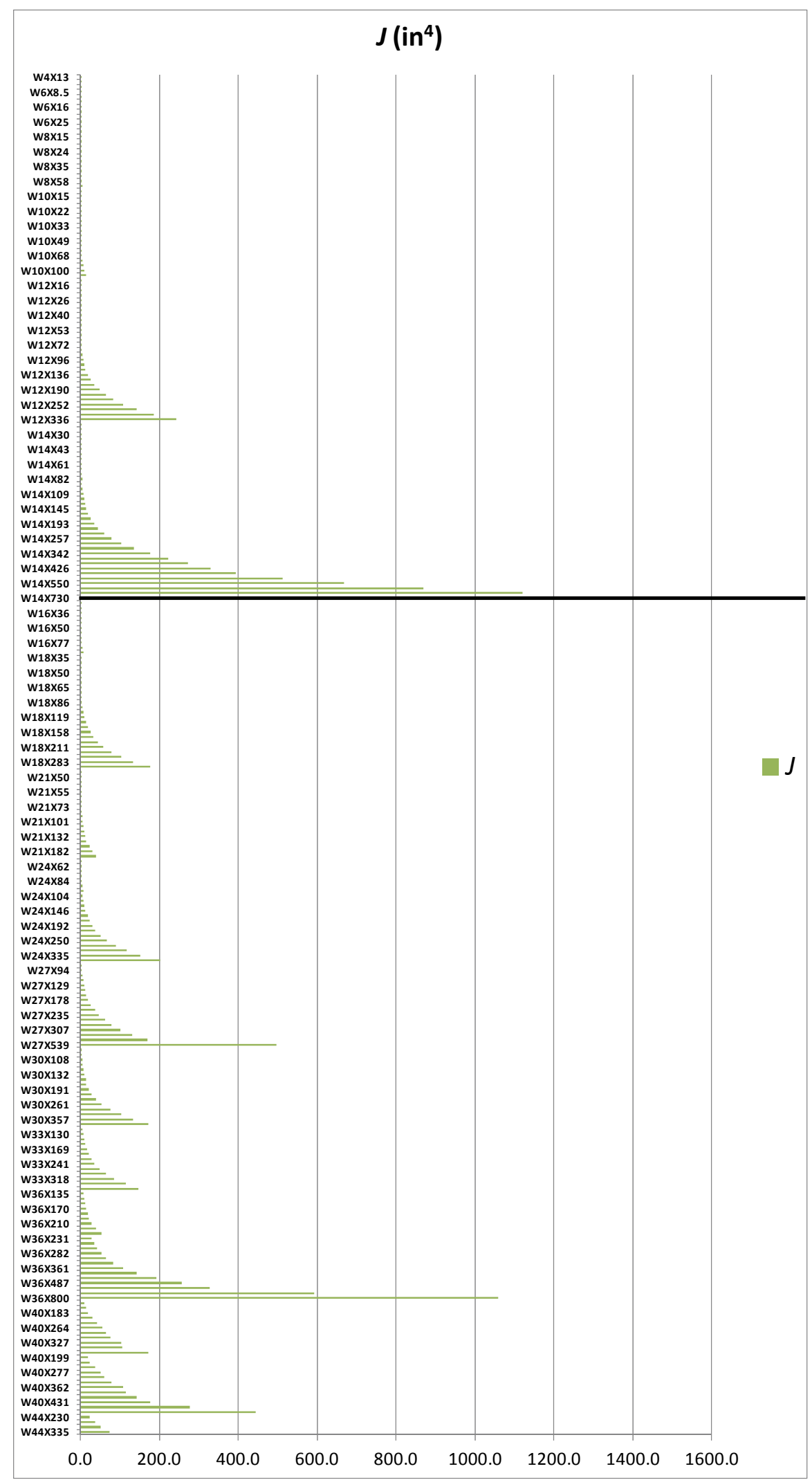

Figure 4-3 Variation in torsional constant, $J$, for wide flange sections ranging from W4 to W44. 
Continuum elements that can model both in-plane and out-of-plane deformations are recommended over shell elements because they can capture torsional geometric and material nonlinearities more accurately, and such behavior is expected to be important for some deep column sections. Large-strain mechanics should be considered for the geometric nonlinear formulation of the elements, particularly to allow post-peak behavior, including reverse cyclic loading after achieving peak response in one direction. A range of member imperfections should be imposed to assess sensitivity of the results to these imperfections, particularly out-of-straightness and out-of-plumbness.

The cyclic steel constitutive model should be sufficient to capture the material behavior of all necessary failure modes. For the bulk of these studies, a comprehensive multi-axial constitutive model should be used. However, there may be some advantages to simplifying the material models (e.g., elastic-plastic) for investigation of behavior assumed for design. A standard residual stress pattern appropriate for deep column sections should be established. It should not be necessary to model fracture in the member analyses. Parametric variation of the magnitude of residual stress would identify sensitivity to the material characteristics of the section.

Quasi-static cyclic analysis should be sufficient for member analyses. The analysis driver should be capable of capturing reverse cyclic loading emanating from all points on a back-bone curve, including points after significant softening, either through a work-control or arc-length procedure. It is important that the analysis driver be able to simulate complex multi-axial non-proportional loading protocols.

\subsubsection{Member Selection}

Member sizes selected for computational research should be chosen to achieve the following:

- Deep sections with both stocky and slender flanges and webs should be chosen to investigate the range of possible damage states, including possible differences in the progression of damage, based on the likelihood of local buckling versus global member failure. ANSI/AISC 341-10 typically requires flanges and webs to be as compact (or more compact) than the criteria in ANSI/AISC 360-10, with the exception of webs in members subjected to axial compression. Members having relatively small as well as relatively large values of cross-section slenderness should be selected. Figures 4-4 and 4-5 plot the variation in flange and web slenderness ratios for wide flange sections ranging from W4 to W44. 


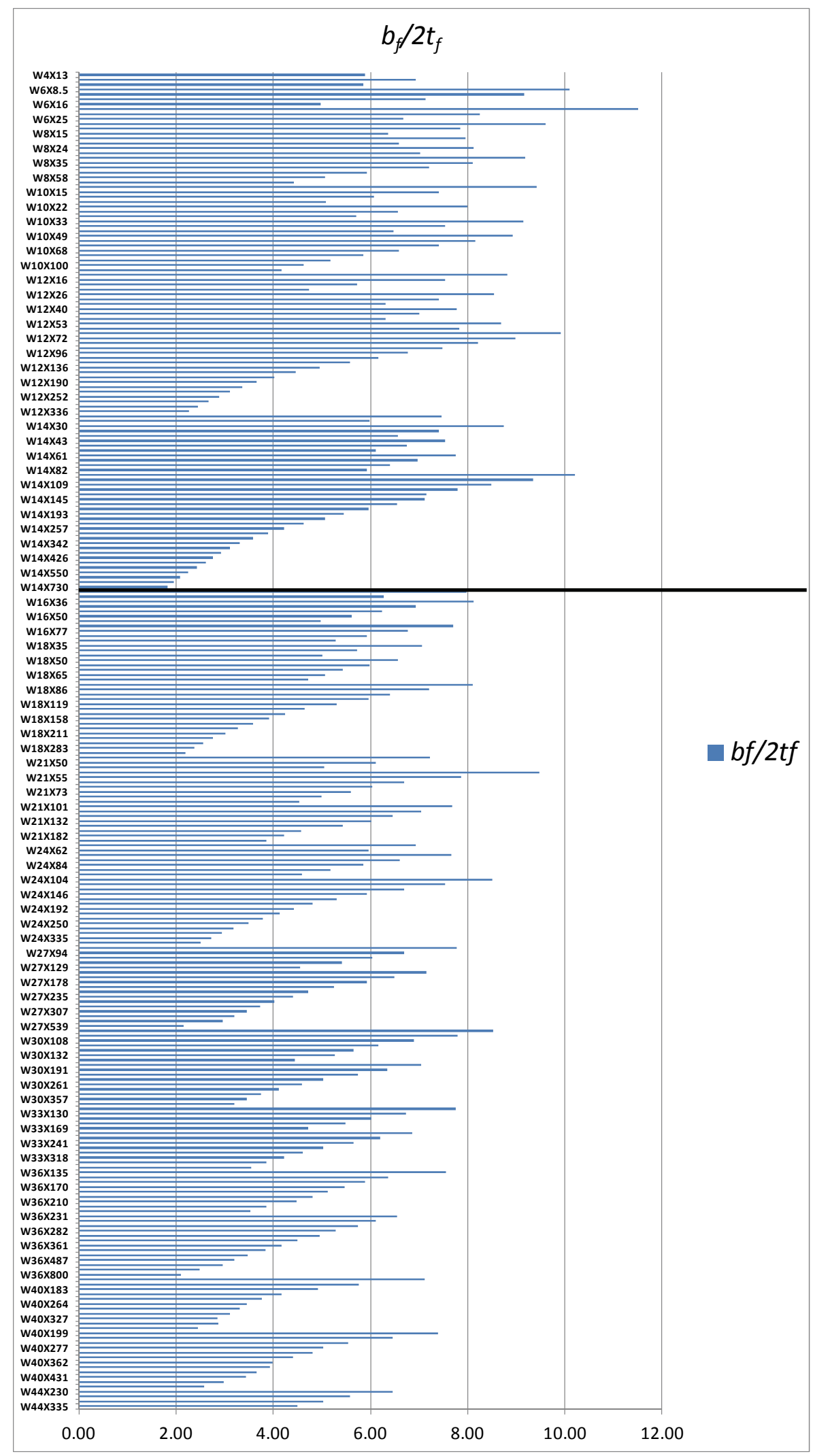

Figure 4-4 Variation in flange slenderness, $b_{f} / 2 t_{f}$, for wide flange sections ranging from W4 to W44. 


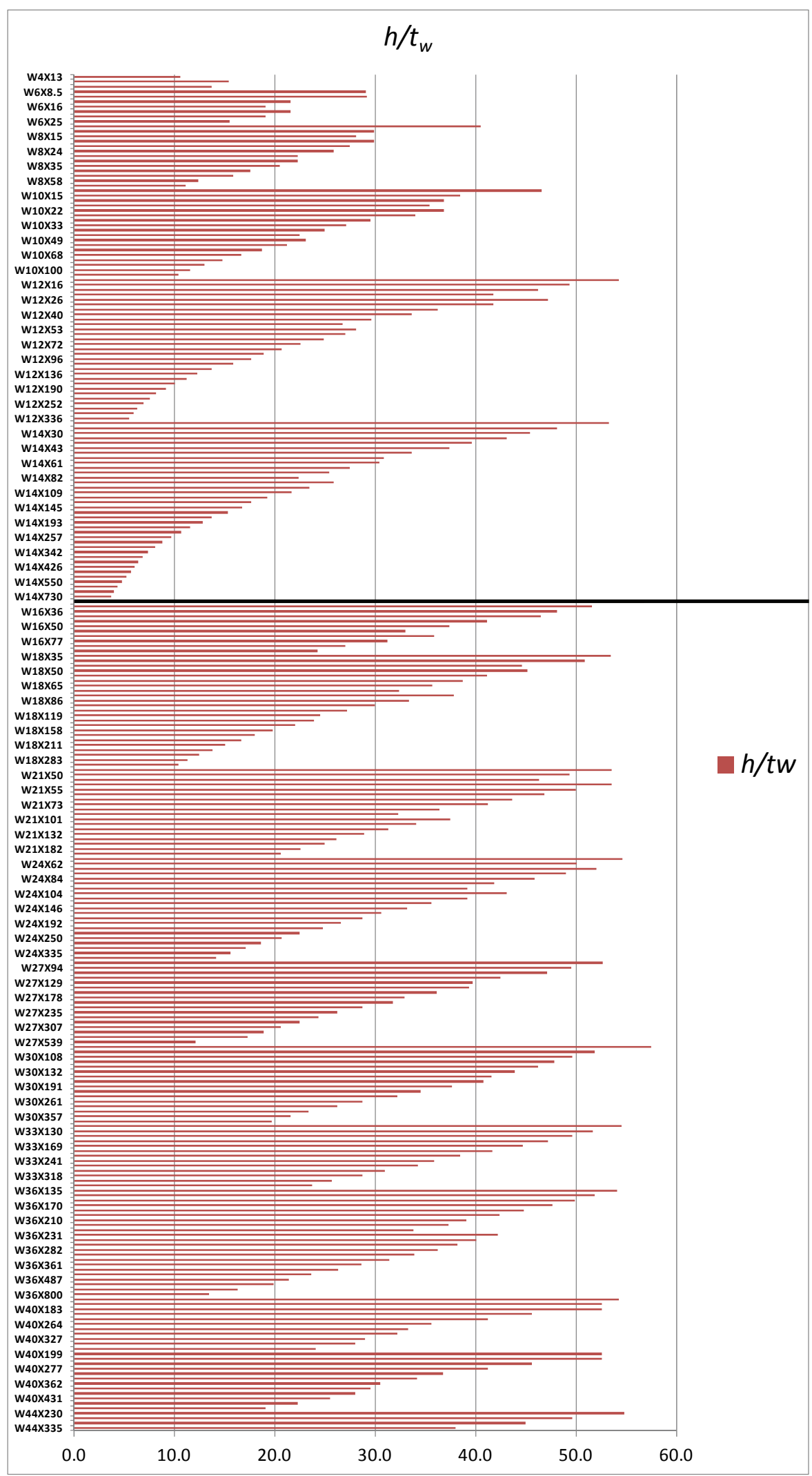

Figure 4-5 Variation in web slenderness, $h / t_{w}$, for wide flange sections ranging from W4 to W44. 
In Figure 4-4, few rolled shapes W16 and larger have a flange slenderness ratio, $b_{f} / 2_{t}$, that exceeds ANSI/AISC 341-10 limits for moderate ductility, which are $b_{f} / 2_{t f}<8.0$ for Grade 65 material, and $b_{f} / 2_{t f}<9.2$ for Grade 50 material. Several shapes, however, have a flange slenderness ratio that exceeds the limits for high ductility, which are $b_{f} / 2_{t f}<6.3$ for Grade 65 material, and $b_{f} / 2_{t f}<7.2$ for Grade 50 material.

Web slenderness limits in ANSI/AISC 341-10 vary with axial load ratio. In Figure 4-5, no rolled shapes W16 and larger have a web slenderness ratio, $h / t_{w}$, that exceeds a limit of $h / t_{w}<60.0$ for moderate ductility of Grade 50 material under high axial load. Several shapes, however, have a web slenderness ratio that exceeds a limit of $h / t_{w}<45.6$ for high ductility of Grade 65 material under high axial load. The use of Grade 75 material can be considered for probing these ductility limits.

- Members that are controlled by different member limit states identified above should be selected to track differences in the progression of damage between these limits.

- Member sizes should be selected considering the likely peak load and displacement limits of available testing facilities so that some member sizes that are studied computationally are also likely to be tested experimentally.

- To the extent possible, selected member sizes should complement member sizes used in the subassemblage and system elements of the research program to facilitate comparison of response.

\subsubsection{Boundary Conditions and Loading Protocols}

Boundary conditions used in computational research should bracket the range of possible boundary conditions, in three dimensions, that can be experienced by deep beam-columns in practice. It is important to consider a range of flexural and torsional boundary conditions to ensure that flexural buckling, flexural-torsional buckling, and lateral-torsional buckling are adequately represented by current AISC equations. Allowing both torsionally fixed and torsionally free boundary conditions, as well as rotationally fixed and rotationally free boundary conditions, and understanding the range of behavior between these extremes, is necessary.

Loading protocols should include both monotonic and cyclic, and both proportional and non-proportional loading. Typically, displacement values should be used to establish the loading protocols. Examples of monotonic-loading protocols that should be considered include:

- Pure axial compression to discern the axial strength for the effective length factor established by the boundary conditions. The residual stress pattern will be an 
important parameter for this loading. Local buckling patterns should be identified as well as flexural buckling failure.

- Pure flexure (with a focus on strong-axis flexure) to discern flexural limit states, including combined local and lateral-torsional buckling for deep column members.

- Axial compression, at varying levels, followed by uniaxial or biaxial flexure, to establish the interaction strength of a member. Flexural loading should be sufficient to induce various failure modes ranging from plastic hinge formation to lateral-torsional buckling. Assessment of the effect of axial compression on the flexural response should be explored thoroughly, as this will help to assess not only the accuracy of the member interaction equations, but also the accuracy of strong-column-weak-beam provisions and panel zone strength. Application of axial tension should be included, as it is possible for end columns in moment frames to experience tension.

- Proportional loading to induce axial compression plus uniaxial or biaxial flexure may be considered to compare to non-proportional loading cases.

These loading protocols will help to establish the variation in strength and stiffness response of the members as they fail, including documentation of the ductility of the members. As such, analyses should be taken well past the peak load (e.g., until the load at peak displacement dips below approximately $50 \%$ of the peak load), if possible.

Examples of cyclic-loading protocols that should be considered include the following:

- Load Combinations. Loading patterns that include combined axial compression and uniaxial or biaxial flexure (e.g., applying a figure-eight pattern of orthogonal displacements non-proportionally to induce biaxial flexure while holding the axial force constant). Application of axial tension should be considered. Varying both axial force as well as flexure in some of the loading protocols is important to simulate the potentially large changes in loads at end columns in moment frames. Loading protocols may be developed based on patterns established in ANSI/AISC 341-10. A minimum of two cycles should be performed at each load or displacement level. The effect of axial compression on cyclic flexural response should be assessed to determine the potential influence on strong-column-weak-beam provisions and panel zone strength, each of which includes reductions in strength for axial compression.

- Torsional Loading. By applying cyclic torsional loading or rotation, it can be established whether there is a severe degradation in torsional strength under cyclic loading. Torsional rotations may be gradually increased, with a minimum 
of two cycles at each level. In addition, loading protocols that combine axial force, flexure, and small to moderate amounts of torsion may be especially important for deep end columns of moment frames, and should be explored to determine possible interdependencies in the resulting behavior.

- Constitutive Validation. In order to facilitate the formulation of new beam finite elements that may be more accurate for deep beam-column members, a secondary set of loading protocols may need to be considered in which a series of probes are conducted to induce biaxial flexure plus compression in order to document the shifting size, position, and shape of the yield surfaces (identifying the limit point behavior).

\subsubsection{Simulation of the Experimental Program}

Prior to implementation of the experimental program, direct simulation of the proposed experiments should be conducted using analysis parameters similar to those described above. Member sizes, boundary conditions and loading protocols should replicate those planned in the experiments. In addition, the likely location of instruments used to measure experimental response should be coordinated with the locations used to assess member response in the simulation analyses. Through such analyses, the anticipated range of performance in the experimental plan can be investigated to ensure that the objectives of the research program are being met.

\subsubsection{Assessment of Computational Research Results}

The following behaviors should be investigated and documented as part of the computational research plan:

- Progression of damage in deep beam-column members, particularly the extent of yielding prior to buckling.

- Reasons for, and extent of post-peak strength degradation, and characterization of member ductility.

- Sensitivity of the response and progression of damage to section and member slenderness parameters.

- Member strength as compared to current AISC formulas, including formulas for interaction strength (including the strength of individual modes of behavior), local buckling limits, and bracing limits. Assessment of the debilitating effects of axial compression on flexural strength should be considered relative to current provisions for strong-column-weak-beam design.

\subsection{Experimental Research Plan}

Once the behavior of deep beam-column members is computationally understood, the experimental research program can be developed. 


\subsubsection{Experimental Strategy}

As deep beam-column members are often governed by three-dimensional failure modes, it is important for the experimental program to carefully consider the boundary conditions for six degrees-of-freedom at each end of the test specimens. The same failure modes investigated in the computational research plan are relevant for investigation in the experimental plan. With this type of complexity, significant instrumentation should be used, including, if possible, instrumentation that can capture three-dimensional failure modes. Member imperfections should be measured prior to the experiment, including out-of-straightness and out-of-plumbness.

\subsubsection{Member Selection}

Member sizes selected for the experimental program should build off sizes used in the computational research plan. These should be based on representative sizes for deep beam-column members used in archetypical building designs, and should also consider sizes chosen for the subassemblage and system experimental programs. Members should be selected to ensure that a comprehensive range of failure modes and damage progressions can be investigated, as established by the computational study. Sizes must also be selected to ensure failure within the load and stroke limits of the testing apparatus. When combined with variations in boundary conditions and loading protocols, it is estimated that on the order of 15 to 30 member tests should be considered.

\subsubsection{Boundary Conditions and Loading Protocols}

Boundary conditions selected at the ends of each specimen should bracket the range of possible boundary conditions for deep columns in three dimensions, based on the sensitivities and failure modes observed in the computational study. It is important to consider a range of flexural and torsional boundary conditions to ensure that flexural buckling, flexural-torsional buckling, and lateral-torsional buckling are represented adequately by current AISC equations. Allowing one end freedom to rotate and twist is a necessary requirement for this experimental program. In order to fully assess lateral-torsional and flexural-torsional buckling behavior, as well as potential distortion of the cross-section, it would be beneficial if, for some tests, both ends were free to rotate as well as twist.

Additionally, the introduction of intra-span loads or moments (force couples) should be explored to simulate boundary conditions due to intervening floor levels. This will enable a different set of rotational boundary conditions to be established along the length of the specimens than would otherwise be possible with a baseplate boundary at one end, and will enable exploration of limit states for a variety of unsupported lengths and moment gradients in the presence of axial compression. 
Loading protocols should include both monotonic and cyclic, and both proportional and non-proportional loading. Monotonic- and cyclic-loading protocols should be reflective of those used in the computational research plan outlined above.

\subsubsection{Assessment of Results}

The following behaviors should be investigated and documented as part of the experimental research plan:

- Progression of damage in deep beam-column members, particularly the extent of yielding prior to buckling.

- Reasons for, and extent of post-peak strength degradation, and characterization of member ductility to determine which members are sufficiently ductile to be classified as moderately or highly ductile (in comparison to the current AISC limits).

- Premature fractures (e.g., due to cyclic local buckling).

- Experimental member strength as compared to current AISC formulas, including formulas for interaction strength, strength of individual modes of behavior, local buckling limits, and bracing limits. Sensitivity to boundary conditions and member imperfections should also be considered.

\subsection{Corroborating Computational Research}

Corroborating computational research should be performed. Corroborating analyses should be focused on simulating the experimental tests, including boundary conditions and loading protocols. Responses should be calculated in a manner that is comparable to the associated experimental measurements. Comparisons with experimental results will then be able to highlight details of member behavior. This can, in turn, lead to additional parametric variations in computational study to probe additional limits on the behavior of these members. Corroborating computational research should use the same analysis methodologies outlined in Section 4.3.

Objectives of corroborating computational studies include:

- Isolating whether yielding, or local and/or member buckling precipitates the failure in each experiment, and validating the progression of damage.

- Isolating whether deep columns are more sensitive to member imperfections or distortion of the cross-section than is typically accounted for in design.

- Documenting the reasons for post-peak strength degradation.

- Establishing whether typical constitutive formulations for beam finite elements are adequate for deep beam-column members. 
In addition, corroboration between experimental and computational results can form the basis for the development of new beam finite elements or associated constitutive models appropriate for deep beam-column members. This may be especially relevant if deep columns exhibit more severe strength degradation or out-of-plane instability than stockier columns.

\subsection{Development of Improved Design Recommendations}

Experimental and computational results should be assessed relative to current AISC provisions to determine whether the current equations are sufficient for strength, prediction of failure modes, deformation capacity, and ductility. Most of the limit state equations for wide flange sections were developed for relatively stocky members (e.g., W8 to W14 sections). While, in all likelihood, most of these limit state equations are appropriate for deep columns, modes of behavior specific to deep columns may require a more refined design strategy to ensure safety in high seismic zones. Deeper webs, for example, may cause more significant distortional buckling of the section, which then degrades more quickly during cyclic loading. Larger $r_{x} / r_{y}$ ratios may also lead to premature out-of-plane failure after in-plane forces precipitate significant inelasticity during early stages of cyclic loading.

Equations specific to seismic design provisions should also be assessed, including limits for moderate and high ductility as they apply to deep columns, as well as design provisions related to columns and column splices. Capacity design provisions for moment frames should also be assessed to ensure their appropriateness for deep beam-column members in moment resisting frames. 


\section{Chapter 5}

\section{Research Plan for Studying Subassemblage Behavior}

This chapter describes a recommended plan for studying the behavior of deep, slender wide flange beam-columns in story-based subassemblages. It outlines program objectives and necessary computational and experimental strategies for investigating subassemblage test specimens as part of the eventual development of practice-oriented analytical tools and improved design provisions.

\subsection{Objectives for Subassemblage Investigations}

Subassemblage research clearly lies between member-based and system-based research. Testing and analytical evaluation of subassemblage specimens is simpler and more economical than system-based research, but it is more complex and expensive than member-based research. Objectives of subassemblage investigations are focused on evaluation of story-based behavior and the interactions between beams, columns, and beam-to-column connections. Subassemblages consist of beam, column, and connection assemblies in T-shaped, cruciform, or multi-bay configurations, as shown in Figure 5-1. Distinct from system-based investigations, however, subassemblages do not include multi-story configurations.

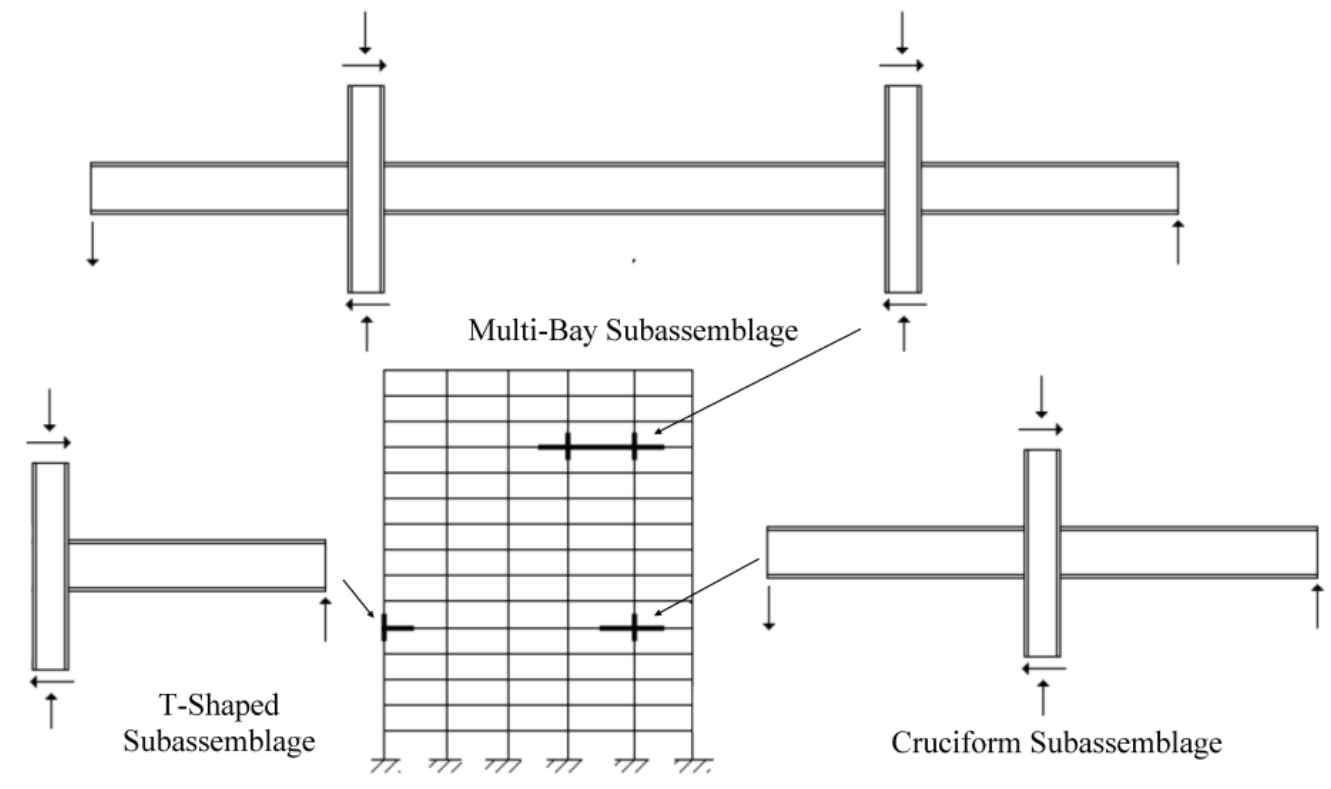

Figure 5-1 Subassemblage configurations recommended for story-based research. 
Subassemblage testing generally employs predetermined boundary conditions, which can limit the ability to accurately evaluate certain behaviors. These boundary conditions and limitations must be fully understood if subassemblage results are to be combined with other research. While subassemblage investigations can address many of the issues concerning deep column moment frame behavior, there will also be considerable overlap and coordination with member and system elements of the overall research program.

\subsection{Computational Research Plan}

A review of available research reveals relatively little data specifically related to deep, slender beam-column behavior. The computational research plan is intended to address these issues by providing a better understanding of the findings from past research results, and by extending these results to address questions that have not yet been explicitly studied. As a result, computational research tasks will facilitate the use of a large body of existing experimental data in an economical manner, and will provide direction and guidance for the experimental research efforts that follow.

\subsubsection{Perform Continuum Nonlinear Analysis of Moment Frame Subassemblages}

As the highest priority, the computational research plan includes detailed nonlinear continuum analyses of subassemblages to evaluate local and global behavior using computer programs such as ANSYS, ABAQUS, LSDYNA or MARC, for example. Shell or three-dimensional solid elements should be utilized in these analyses with a formulation and associated constitutive models able to simulate the full cyclic inelastic behavior of the specimens. Models should include the stiffness and resistance of slabs, lateral support and restraint, and other factors needed to accurately simulate the boundary conditions affecting inelastic performance. The goal of these analyses is to accurately predict both global and local behavior, including estimation of the specimen stiffness, reactions at the boundary conditions and restraint applied to the specimen, prediction of initiation of buckling and yielding, estimation of the magnitude of buckling and out-of-plane deformation, and determination of the overall accuracy of the simulated response and predicted failure modes for the system. As envisioned, this task will continue for the duration of the subassemblage research program, and can be divided into three parts.

Part 1. Part 1 consists of analytical models that are developed to accurately simulate the full range of test results from the SAC Joint Venture Project, and can begin at the start of the subassemblage research program. The SAC Joint Venture Project was a multi-phase, multi-year effort of experimental and analytical investigation on the performance of steel moment resisting frame connections (FEMA, 2000a). The SAC testing program has been targeted because of the significant volume of available of data that can be used to develop, calibrate, and verify the accuracy of nonlinear 
analysis models, and because these data are well-documented in past reports with detailed descriptions of measured material properties, dimensions, test procedures, yield mechanisms, and failure modes including local and global behaviors. Models should be developed both with and without composite slabs. Analyses should focus on reduced beam section (RBS), welded unreinforced flange-welded (WUF-W), bolted flange plate (BFP), and extended end plate (EEP) connections, because these connections have generally achieved good system performance, are prequalified in ANSI/AISC 358-10, and represent a wide range of connection behaviors.

Analytical models will be developed and then calibrated to experiments performed on these specimens to improve the models and to establish their accuracy over the observed range of performance. Analyses must clearly show differences in performance such as the differences between specimens that are laterally braced and unbraced (as illustrated in Figure 2-2) and the differences between specimens with different boundary conditions (as illustrated in Figure 2-3).

Results will illustrate the accuracy that can be achieved by this analytical approach and the modeling necessary to achieve it. The model should permit evaluation of the effect of composite slabs, and should permit assignment of numerical values to the stiffness and resistance of the restraint and lateral support applied to the specimens in past tests. This information will be useful to system-based research, allowing better and more accurate utilization of past experimental research in evaluating system performance. It will also permit better extrapolation of subassemblage test results to system performance.

Part 2. Part 2 consists of a parametric study that is intended to provide focus and guidance to the experimental research program to follow. It should be performed partly in parallel with Part 1, and fully completed immediately following Part 1. The parametric study should consider the following important parameters:

- Variation in beam and column size and depth

- Variation in connection type

- Different $d / t_{w}$ and $b / 2 t_{f}$ ratios for beams and columns

- Effect of composite slabs, and restraint provided by the slab

- Effect of continuity and doubler plates

- Relative importance of different levels of panel zone reinforcement and yielding

- Effect of composite slabs on panel zone deformations

- Effect of different test boundary conditions

- Effect of different stiffness and resistance of lateral supports and/or restraints applied to the specimen 
This study is intended to economically evaluate many parameters and provide focus and direction for the more expensive experimental testing program. It will provide initial estimates of the veracity of various test setups, and aid in establishing priorities for the overall research program. It will be a major tool in coordinating subassemblage work with other elements of the research program, since the models developed in this study will need to interface with member- and system-based research.

Part 3. Part 3 consists of ongoing analytical study in support of the experimental research program. Analyses will be performed on each specimen that is tested to continually improve and validate numerical models and the accuracy of their predictions. This work will be performed as part of the corroborating computational research effort, and will be concurrent with the experimental testing program.

\subsubsection{Establish the Accuracy and Reliability of Panel Zone Predictions}

There are diverse opinions on the role that panel zone yielding plays in moment frame connection performance, and many experiments have been performed. Models have been developed to predict resistance and deformation of panel zones, and there is significant variation in the accuracy and reliability of these predictions. The root cause of this variation is the development of models based largely on limited sets of experimental data from individual researchers with little consideration of data from other tests. To address this issue, all past experimental data, with varying levels of panel zone yielding, will be assembled into a single database. Data in this database must be comprehensive, including:

- Test dimensions and configuration, including actual member sizes, continuity plates, and doubler plates

- Measured material properties for all members and plates

- Measured resistance, inelastic deformation, and general hysteretic behavior

- Accurate information on test setup and loading protocol

- Observed behaviors including sequence of yielding and failure modes

- Other key parameters

Similarly, all available models for predicting inelastic panel zone stiffness and inelastic deformation will be collected into a second database. Through analysis, combination, and comparison of available data with available models, the following questions will be addressed (and assessment of panel zone behavior will be improved):

- How much inelastic deformation can be achieved through panel zone yielding? Is more or less connection ductility achieved with weak or balanced panel zones? 
- Which of the current models work best? Are there conditions where one model is better than others?

- Does excessive panel zone yielding cause reduced inelastic deformation of the connection through premature failure or unexpected failure modes?

- How effective are doubler plates? Do the stitching requirements and the slenderness of the doubler plate and column web influence this behavior?

It is recommended that this task be performed early in the program so that it can provide guidance to the experimental research program. Experimental results can then be used to either confirm or dispute the observations from this work.

\subsubsection{Evaluate Fundamental Theories of Lateral Support and Lateral- Torsional Buckling}

There have been hundreds of papers and publications prepared on lateral-torsional buckling. Past work has focused on elastic buckling and has not been generally directed toward the seismic performance of moment resisting frames. A comprehensive literature review of past research is proposed, with the goal of focusing the results toward inelastic lateral-torsional buckling in moment resisting frames. The best available models may be studied or combined to develop improved models and revised lateral support requirements. These improvements can be evaluated in the experimental research that follows.

Investigation of lateral support requirements and lateral-torsional buckling is proposed in support of detailed continuum analysis and experimental research work. As a result, this task is less likely to be immediately successful on its own, and may ultimately be used to build upon member-based analytical research studies. This topic is viewed as no less important than panel zone behavior, but it is expected to start later than other analytical research because existing lateral-torsional research is predominantly elastic.

\subsection{Experimental Research Plan}

Experimental research should be staged to begin after the start of the computational research plan so that design of the experimental research program can utilize the knowledge gained during initial analyses. It should also run in parallel with computational research so that experimental results can inform the remaining analyses. Experimental research teams will have the responsibility to demonstrate that the experimental setup has boundaries and constraints that are clearly defined and well understood. This knowledge will permit more ready use of subassemblage results in system evaluation. It is anticipated that the majority of the tests would be performed using the loading protocol contained in ANSI/AISC 341-10, Appendix S. 


\subsubsection{Initial Testing on Deep Column Subassemblages}

Initial testing on deep column subassemblages will consist of T-shaped specimens to reduce cost. At least 16 subassemblage specimens are envisioned. They should include RBS, BFP, EEP and WUF-W connections, each with multiple column sizes. It is recommended that a baseline case for each specimen should use a W12 or W14 column, which will allow for direct comparison with past SAC test results. Three additional specimens for each connection type should then be prepared using deeper column sections. The deep column sections should consist of a range of flange and web width-to-thickness ratios, from stocky to slender.

It is expected that some specimens will employ normal continuity and doubler plate reinforcement, while others will employ reduced requirements. Reduced continuity and doubler plate specimens will aid in better understanding the effect of these elements on connection behavior, and will interface with other studies such as the panel zone computational research.

The actual details of the test program must be designed based on initial information gained from the computational research plan. The test setup for each specimen must be carefully designed and built so that the boundary conditions of the test and the lateral support provided to the subassemblage are known and understood prior to testing.

It is envisioned that this first experimental study will be initiated after Part 1 of the computational research studies, but will run approximately in parallel with remaining studies. Results from these tests are intended to provide basic guidance on the effect of deep columns on subassemblage behavior. Results will be compared to subsequent computational research to improve analytical models.

It is anticipated that observed performance will lead to grouping of specimen type by performance. A number of potential groupings are possible. For example, BFP and EEP connections may be grouped because they are both field bolted connections. However, BFP connections yield at some distance from the face of the column and may have different lateral-torsional deformation behaviors than EEP connections. Considering lateral-torsional buckling, grouping of RBS and BFP connections may also be logical. Grouping decisions will be made based on the observed performance and similarities of performance within the group. Grouping of specimens by performance is expected to help guide future testing and provide greater economy in the testing that follows.

\subsubsection{Investigation of Slab Effects, Lateral Support, and Selected Design Parameters}

Based on the observed performance of initial subassemblage tests, two groups of specimens will be selected for additional testing to investigate the effects of slabs, 
lateral support, and continuity and doubler plates on the performance of moment resisting frames with deep columns. For this more detailed study, subassemblages will consist of cruciform specimens and many will have composite floor slabs. It is recommended that most specimens with floor slabs consist of multi-bay subassemblages to better capture the boundary conditions present in real structures.

It is estimated that at least 12 multi-bay subassemblage specimens will be tested in this series, and they will be divided into performance groupings identified during the initial series of tests on T-shaped subassemblages. The first specimen in each group will be a baseline specimen without a composite floor slab to allow direct comparison with the initial series of tests. Subsequent specimens will have different levels of lateral support, column web and flange slenderness, continuity plates, and panel zone reinforcement.

Details must be designed based upon information gained from the computational research plan, and the test setup for each specimen must be carefully conceived so that the boundary conditions and lateral support provided to the subassemblages are known and understood prior to testing. Results from these tests are intended to provide a more comprehensive understanding of deep column behavior, the effects of slabs and lateral restraint on deep column systems, and the effects of key design parameters related to continuity and doubler plates. Results should be correlated to earlier experimental research and the results of ongoing computational research tasks.

\subsubsection{Investigation of Additional Issues}

A third experimental series is proposed to address: (1) axial load effects; (2) cyclic loading protocol effects; and (3) additional questions arising out of the subassemblage computational and experimental research work. In doing so, it is envisioned that at least 12 additional cruciform or multi-bay subassemblages will be needed across two performance groupings selected from the initial series of tests.

Axial load. Prior test series were proposed herein without any axial load. This was not intended to suggest that axial load is not important. However, it is recognized that deep columns may be less attractive candidates for columns with larger axial load, because deep sections with narrow flanges are more likely to be controlled by weak-axis buckling. Further, axial load effects are more strongly related to system performance issues.

Weak column behavior (i.e., weak relative to beams) is affected by two factors. One factor is a system-level concern in that inelastic deformation becomes concentrated into a single story. A second factor is a member-level concern in that damage to a column accumulates quickly if the axial compression exceeds about $0.5 P_{y}$, and accumulates more slowly if the axial compression is less than about $0.3 P_{y}$. 
A minimum of two subassemblage specimens in each of two performance groups are recommended for testing with axial load. Axial load ratios in the range $0.25 P_{y}$ to $0.5 P_{y}$ appear to be appropriate initial recommendations for these tests. Tests should consider variations in the axial load with fixed column size, and specimens should be designed with deliberately undersized columns based on SCWB design provisions. The number of specimens could be expanded to consider axial load effects on each connection type. In this case, eight specimens should be considered, with axial load ratios of $0.25 P_{y}$ and $0.5 P_{y}$ for each connection type.

Cyclic-loading protocol. Prior test series used the ANSI/AISC 341-10 testing protocol. However, near fault acceleration records cause very different cyclic load histories. A few past experiments have evaluated near fault loading protocols. Generally, inelastic performance has not been dramatically impacted by this test protocol. As a result, one specimen in each group is recommended for testing using a near fault protocol. Limited monotonic testing may also be considered to partially address this issue.

Subduction zone earthquakes are important in the Pacific Northwest, and these earthquakes can cause very long duration strong shaking, although the peak acceleration is expected be somewhat smaller than other near fault events because the likely epicenter of such earthquakes is offshore. Limited testing has been done on moment frames subjected to this type of seismic loading, and this represents another potential cyclic-loading protocol to be considered.

Additional issues. It is anticipated that additional questions will arise from computational and experimental research conducted on subassemblages. As a result, it is expected that additional specimens will be developed to address specific questions and issues raised in the work conducted to this point.

\subsection{Corroborating Computational Research}

The primary corroborating computational research effort consists of ongoing analytical studies in support of experimental research that is described under Part 3 of Section 5.2.1. Additional corroboration is achieved through comparison and calibration of subassemblage results to system-level computational and experimental research, and by showing that story-based subassemblage research can be used directly in the evaluation of system performance.

Confirmation testing and computational analysis of system-level specimens will be the strongest form of verification of subassemblage results. It is therefore recommended that at least one test (and the accompanying analysis) in the systembased element of the overall research plan be dedicated to confirming how well member- and story-based research results compare with system-based performance. 


\subsection{Development of Practice-Oriented Analytical Tools and Improved Design Recommendations}

Analytical tools envisioned under the computational research plan will be valuable research tools, but their complexity and likely expense to implement are expected to preclude them from becoming useful tools in engineering practice. As a result, a concerted effort is recommended to develop practice-oriented analytical tools for use in design practice. This work will utilize the results of all research tasks and will develop models that best predict both global and local response of moment resisting frame systems. Such models may consist of line element models or fiber models, which are becoming increasingly efficient and practical for analyzing large systems. Fundamental questions such as the use of concentrated plastic hinges, hinge length, and similar approaches will be considered. Multiple modeling methods that offer different benefits may be proposed. The accuracy at both the global and local level will be established through comparison of computational results to experimental results.

Experimental and analytical results from subassemblage specimens should be evaluated and assessed relative to current design provisions related to flange and web slenderness limits, lateral support requirements (stiffness and resistance), strongcolumn-weak-beam limitations, continuity plates, and panel zone reinforcement. Recommendations for any improvements will be developed, subject to confirmation from member-based and system-based research results. 



\section{Chapter 6}

\section{Research Plan for Studying System Behavior}

This chapter describes a recommended plan for studying the behavior of deep, slender wide flange beam-columns in special moment frame (SMF) systems. It outlines program objectives and necessary computational and experimental strategies for investigating system-level specimen performance as part of the eventual development of improved design provisions.

\subsection{Objectives for System-Level Investigations}

System-level investigations are intended to verify behavior of individual members and connection assemblies within the context of the entire moment resisting frame system. Member and subassemblage specimens are limited in that boundary conditions representing the potential interaction with other elements in the system must be imposed. This is accurate to the extent that these interactions are known. System-level investigations will be used to confirm that boundary condition assumptions are valid. At the same time, information gained from member and subassemblage investigations will be used to improve the accuracy and robustness of system-level simulations.

\subsection{Computational Research Plan}

The computational research plan consists of two parts: preliminary computational studies, and advanced computational studies. Preliminary computational studies will be conducted on archetype buildings described in Chapter 3, and will use state-ofthe-art simulation models that have been calibrated based on currently available information from past analytical and experimental studies. Advanced computational studies will narrow the list of important design variables considered in the archetype buildings. Models will be expanded to three-dimensions and updated with refined simulation models calibrated with updated information on composite beams, panel zones, column bases, and gravity framing.

System-level analytical work will consist of nonlinear static analyses and nonlinear response history analyses for evaluation of global behavior. A number of research analysis programs are available and could be used for this purpose, including OpenSees, Open System for Earthquake Engineering Simulation (McKenna, 1997), and FRAME3D, for example. The OpenSees simulation platform is also compatible 
with the Open-Source Framework for Experimental Setup and Control (Schellenberg, 2008), which could be utilized for hybrid simulation tests that involve member, subassemblage, and system level experimental tests.

\subsubsection{Preliminary Computational Studies}

Preliminary computational studies will be conducted using the archetype buildings described in Chapter 3. Archetype buildings will be analyzed with state-of-the-art simulation models that have been calibrated based on currently available information from past analytical and experimental studies (see Appendix A).

Preliminary system-based computational research will contribute to the identification of high priority issues that affect the behavior of moment frame systems using deep columns. This effort is envisioned to be "dynamic," which implies that the analytical models will be updated with information from member and subassemblage research as it becomes available. Preliminary computational studies are expected to:

- Provide a range of representative axial load ratios for experimental testing of members and subassemblages.

- Identify which of the archetype design variables summarized in Chapter 3 have the most significant impact on the behavior of moment frame systems with deep columns.

- Identify limitations in currently available standards and guidelines for modeling steel components.

- Address limitations in state-of-the-art simulation models for capturing the postbuckling cyclic behavior of deep columns.

\section{Simplified Models}

A number of critical issues related to dynamic response can be identified with simplified building models as a first step. In particular, two-dimensional, one-bay models have been utilized to investigate system-level response parameters by varying a number of design variables such as: (1) the strong-column-weak-beam (SCWB) ratio; (2) the number of stories and bays; (3) second-order (P-Delta) effects; and (4) the effect of gravity framing (NIST, 2010). The advantage of using simplified models is reduced computational effort. Simplified models can also be used to evaluate ground motion input parameters and investigate dynamic instability for different ground motion sets.

Simplified modeling recommendations for steel structural components will be based on PEER/ATC 72-1, Modeling and Acceptance Criteria for Seismic Design and Analysis of Tall Buildings (PEER/ATC, 2010), and ASCE/SEI 41-06, Seismic 
Rehabilitation of Existing Buildings (ASCE, 2007). These component models are illustrated and compared in Figure 6-1.

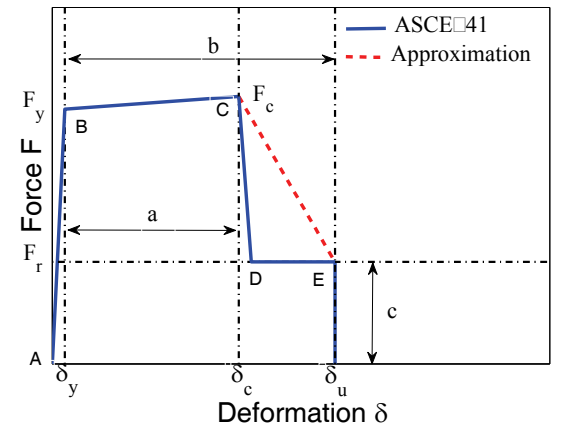

(a)

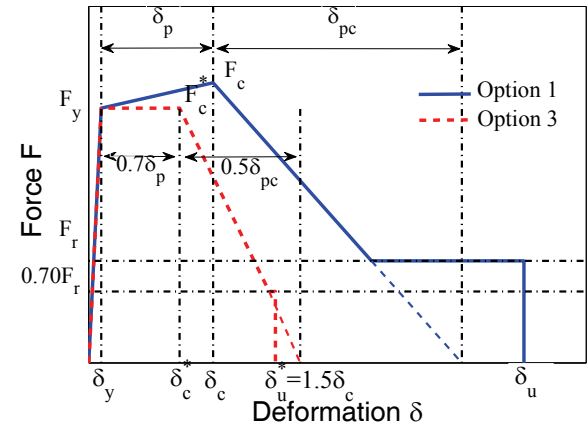

(b)

Figure 6-1 Comparison between ASCE/SEI 41-06 and modeling options based on PEER/ATC-72-1 (Lignos et al., 2011e).

PEER/ATC 72-1 does not include comprehensive information on deep, slender columns due to lack of experimental data, and ASCE/SEI 41-06 contains somewhat conservative values for steel members with high axial load ratios (see Newell and Uang, 2006). A high priority objective is to improve these two sources for modeling and verification of steel components subjected to axial load. Emphasis will be placed on how to address three-dimensional effects (interaction of axial load and biaxial bending) in a simplified manner for component modeling of deep columns. This issue is also related to three-dimensional motion and torsional effects and must be coordinated with member-based research results.

\section{Building Prototype Models}

Building prototype models are full-building nonlinear models developed from the archetypes buildings discussed in Chapter 3. These detailed models will be used to explore parametric variations on building characteristics and their effect on response. Two-dimensional and three-dimensional models of the archetype buildings will be developed. Three-dimensional models are necessary for considering threedimensional effects including coupling of axial load and biaxial bending at end columns, out-of-plane stability of deep beam-to-column connections, and the effect of the gravity framing on dynamic response. However, due to lack of available experimental information on three-dimensional effects, preliminary computational studies will emphasize two-dimensional models. Modeling of slab effects, panel zone response, column bases and splices is essential.

Nonlinear building prototype models will incorporate strength and stiffness deterioration of beams and columns and must account for second-order effects. State-of-the-art component simulation models that have been calibrated with available experimental data from past experimental studies will be employed for this purpose. For steel beams, emphasis will be placed on research that includes beams 
with reduced beam sections (RBS), welded unreinforced flange-welded (WUF-W) and bolted flange plate (BFP) connections. It is also essential that component simulation models be validated with recent small-scale and full-scale shake table tests (Suita et al., 2008; Lignos et al., 2011a; Lignos et al., 2011d).

Two types of nonlinear archetype building models will be developed. The first will include the bare steel frame only (i.e., no consideration of the composite action and gravity framing). The second will incorporate the slab and other elements in order to quantify the effects of additional restraint. Preliminary modeling assumptions for this purpose are summarized in Lignos et al. (2011e) and shown in Figure 6-2. A distinction will be made for composite beams that are part of interior or exterior connections.

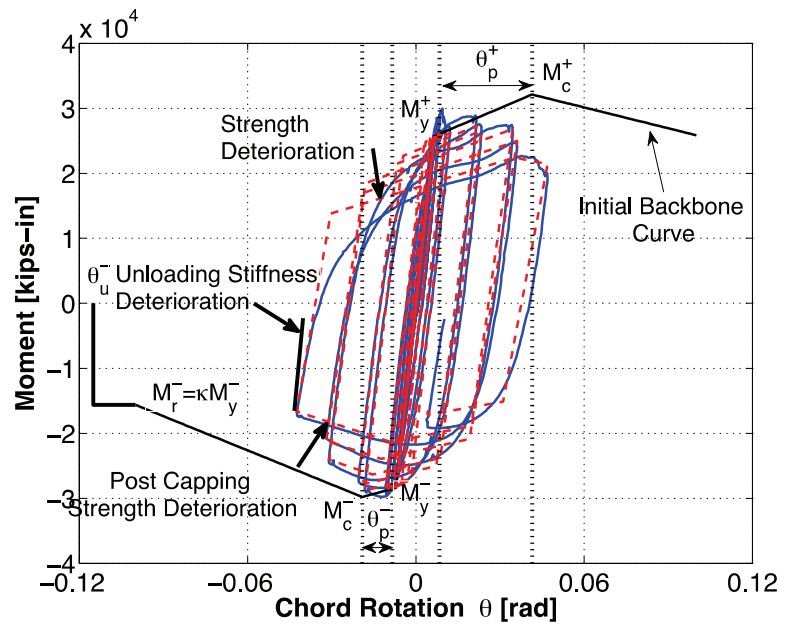

Figure 6-2 Calibration of the deduced moment rotation diagram of a composite beam with RBS (Lignos et al., 2011e).

Coupling of axial load and uniaxial/biaxial bending including cyclic deterioration will be modeled in order to predict the post-buckling cyclic response of deep columns. Experimental data on slender steel columns are limited. Thus, available finite element simulations such as those employed by Newell and Uang (2006) may be used as a starting point for calibration. This is a high priority issue for reliable assessment of moment frames with deep columns, and should be coordinated with member-based research results.

Detailed nonlinear models will also incorporate explicit panel zone modeling. Available models (Krawinkler, 1971; Wang, 1988; Kim and Engelhardt, 2002; Lee et al., 2003c) will be considered for this purpose. This effort will be coordinated with calibration of panel zone models with experimental data from subassemblage-based research. 
Models should also include explicit consideration of column base connection restraint. A distinction will be made between exposed base plate versus embedded column base connections.

\subsubsection{Advanced Computational Studies}

Advanced computational studies will narrow the list of important design variables considered in the development of archetype buildings. This work will build on the two-dimensional and three-dimensional models that were developed during preliminary computational research studies. Information from integrated computational and experimental research on member and subassemblage specimens will be used to update the analytical models used to simulate member and connection behavior.

Advanced computational studies will consider the following:

- Re-design (if needed) of the archetype buildings based on available design recommendations from member-based and subassemblage-based research.

- Importance of slab effects on cyclic behavior, stability of beam-to-column connections, and dynamic instability moment frame systems. This is a high priority issue that will be addressed with refined analytical models that consider composite action for steel beams based on information from subassemblagebased experimental tests.

- SCWB ratios that provide stability of deep beam-to-column connections and delay or prevent local story mechanisms, particularly in the bottom stories multistory frames. This includes considering the effects of dynamic axial load ratios on SCWB behavior.

- Interaction of specific connection types with deep columns based on the experimental and detailed finite element program for subassemblage-based research.

- Cyclic behavior of deep columns including three-dimensional effects. This requires member-based experimental tests to determine the effect of coupling of axial load and biaxial bending on deep columns. Three-dimensional column models will be developed and calibrated with available experimental data and incorporated into three-dimensional nonlinear archetype models.

- The use of advanced panel zone models that are able to simulate shear buckling in deep columns. This will require extensive calibration with experimental data from past studies and current subassemblage-based experimental research. For panel zone design, material strength variability between the column flange and web, and the additional strength of beams with composite slabs, will be considered. 
- Incorporation of column base modeling in cases where preliminary computational studies demonstrate that this effect is important. This will require calibration with experimental data from member-based tests that include alternative column base conditions.

- Incorporation of gravity framing in the numerical models taking advantage of available information such as that from the SAC Phase II tests related to shear connections (see Liu and Astaneh, 2000). The contributions of gravity framing to lateral stiffness and strength of moment resisting frames will be quantified.

\section{Additional Issues Related to System Performance}

The following additional issues can be related to seismic performance of moment frame systems and should be considered in the computational research plan. It is recognized that these issues may not be as high of a priority as the issues identified above.

- Effect of column splices should be considered for performance evaluation of moment frames with deep columns. As long as splices are designed with complete joint penetration welds, the potential for splice failure is not expected to significantly affect system performance. However, assessment of demands on column splices when subjected to moment and axial load is recommended. This is particularly important for columns that are not part of the seismic-force resisting system (i.e., columns in gravity frames). Calibration of nonlinear models, which will be used for modeling of deep column splices, should be based on member tests with deep columns that investigate how in-plane stability and flexural torsional buckling of slender columns affects column splices.

- Modeling and ground motion uncertainty should be considered for performance evaluation of moment frames with deep columns. To address modeling uncertainties, modeling parameters will be described with complete probability distributions including intra-member and inter-member correlation information (see Lignos and Krawinkler, 2011). For deep columns, missing information will be based on results of member-based and subassemblage-based testing.

\subsection{Experimental Research Plan}

System-level experimental research is intended to investigate issues that cannot be addressed through member-based and subassemblage-based testing alone. Systemlevel specimens consist of full-scale complete frame systems, or subsystems consisting of multi-bay, multi-story portions of a complete frame. As a result, the system-level testing matrix is limited due to the size of the specimens and complexity of the test set-up. 
System-level experimental testing includes the use of hybrid simulation techniques on partial frame specimens, and shake table tests on full-scale complete frame specimens. Ultimately, shake table testing is recommended to confirm the overall integrated results of all elements of the recommended research plan.

System-level experimental testing should be initiated after the completion of all proposed subassemblage tests, and after the completion of system-based computational research. Design of test specimens and test set-up should be informed by the computational research results.

\subsubsection{Hybrid Simulation Tests}

The hybrid simulation technique will be used in the first experimental test series to reduce potential cost. Before utilizing the hybrid simulation technique, past and ongoing experimental studies should be reviewed to assess the potential limitations of this experimental method relative to shake table tests. Relevant studies include Schellenberg (2008), Wang et al. (2010), and Lignos et al. (2011a), as well as the NEES Research project, Collapse Simulation of Multi-Story Buildings through Hybrid Testing, which was initiated in 2009 and is scheduled for completion in 2012.

At least four system-level specimens are envisioned for hybrid simulation testing. These partial frame specimens should each include at least two columns, and should represent the bottom stories of selected archetype buildings described in Chapter 3 . Steel members used in some of these specimens should be nominally identical to selected member-based and subassemblage-based specimens to allow for direct comparison and design verification. A slab should be included in all system-level specimens.

The first series of system-level testing is intended to:

- Quantify the axial restraint provided by a composite slab and the reduction in post-buckling deterioration of beam-to-column connections.

- Demonstrate potential differences between boundary conditions assumed in cruciform and T-shaped subassemblies versus the influence of surrounding elements in the structural system.

- Evaluate the stability of deep columns considering story-level behavior, movable points of inflection, and combined axial load and cyclic deformations.

- Evaluate the effect of column base connections on the general stability of deep columns as part of the first story of an entire system.

- Investigate the effects of panel zone design as part of a system, considering information gained from subassemblage-based research and the effects of web doubler plates and continuity plates. 
- Investigate the interaction of deep beams with deep columns as part of a system, including the stability of alternative deep beam-to-column connections.

- Identify representative SCWB ratios for seismic design of moment frames with deep columns to prevent or delay local story mechanisms.

Testing should include at least one quasi-static test with a symmetric loading protocol to allow for comparison with member-based and subassemblage-based experimental results. Other tests will be used to investigate the effect of loading history on the seismic response of moment frame systems. All system-level specimens will be pushed to large inelastic deformations in order to investigate dynamic response near collapse. Second-order effects must be considered in the analytical portion of the hybrid simulation model since this effect is expected to dominate response at large deformations.

\subsubsection{Earthquake Simulation Tests}

Earthquake simulation testing on full-scale, full-frame specimens is proposed to investigate effects that cannot be addressed through hybrid simulation techniques, and to confirm the integrated results of member, subassemblage, and system elements of the overall research plan. Weight and global overturning will become an issue in full-scale specimens of archetype buildings more than five stories tall. A substructuring technique discussed in Chung et al. (2010) may be employed to simulate the upper stories in lieu of complete frame specimens.

Earthquake simulation testing on complete frame specimens will permit further investigation of three-dimensional effects including:

- Biaxial bending and cyclic drift coupled with axial load at end columns due to three-dimensional motion. This may be critical for deep columns due to limitations in weak axis buckling capacity.

- Validation and identification of limitations for advanced and simplified numerical models and modeling platforms (commercial and research).

- Issues related to diaphragm action, lateral support, and slab effects that cannot be captured using hybrid simulation techniques.

- Higher mode effects.

This test series will include scaled intensities of ground motions that are representative of design level and maximum considered earthquake events in regions of moderate and high seismicity. 


\subsection{Corroborating Computational Research}

Corroborating computational research will be performed using updated analytical models from the advanced computational studies. Models will be updated based on information that becomes available from member, subassemblage and system research. The system-level experimental program will be used to validate and further refine the integrated use of all numerical models for moment frame behavior considered and developed as part of the overall research program.

A major issue for corroboration will be to validate modeling capabilities of systems at large deformations where complex nonlinear response is expected.

\subsection{Development of Improved Design Recommendations}

Member-based and subassemblage-based research elements will each have contributed to the development of recommendations for improved design provisions. The system-level research element is intended to confirm these recommendations through the investigation and confirmation of system behavior.

Upon completion of the overall research plan, improved design provisions will be developed, calibrated, and verified for adoption in seismic design standards and use in seismic design practice for steel moment resisting frames with deep slender beamcolumn members. Improvements are anticipated in design provisions related to:

- Appropriate strong-column-weak-beam (SCWB) ratios

- Design of panel zones for deep beam-to-column connections including continuity and doubler plates

- Design criteria for P-Delta effects to limit story collapse mechanisms

- Lateral support requirements for out-of-plane and lateral-torsional stability of deep column members

- Flange and web slenderness ratios to prevent excessive deterioration due to local buckling of deep column members

- Requirements for beam-to-column connections with deep column members

- Column base connections and appropriate assumptions for anticipated rotational restraint 



\section{Chapter 7}

\section{Research Plan: Summary}

This chapter summarizes the recommended tasks, approximate schedule, and order of magnitude budget for a multi-phase, multi-year experimental and analytical research plan investigating the behavior of deep, slender wide-flange structural steel beamcolumn members.

\subsection{Research Plan Objectives}

The objectives of this research plan are the development of: (1) a comprehensive understanding of the seismic behavior of deep, slender wide flange beam-column members; (2) the ability to quantitatively simulate behavior and assess performance; and (3) the development of consensus guidelines for the design and assessment of deep, slender wide flange beam-column members in seismic applications. Work has been structured in a phased approach to identify high-priority analytical and experimental studies, and those studies judged to be easier to complete based on currently available information. The phased approach also allows for the dissemination of useful interim products while allowing sufficient time for necessary developmental work on longer-term studies.

Although the elements of this plan are highly structured and specifically identified, it is understood that the program will evolve as implementation occurs. Wherever possible during implementation, innovative ideas for new experimental and analytical approaches should be encouraged to resolve technical issues that cannot be addressed with present techniques or could not have been foreseen with present knowledge.

\subsection{Research Plan Overview}

A multi-phase, multi-year effort is needed to complete all recommended research tasks. Work has been split up into three primary research components related to member, subassemblage, and system studies. A preliminary phase of work involves the development of archetype building configurations that will form the basis for the selection of members, subassemblages, and systems that are studied. A follow-on phase of work involves the finalization of recommendations and products once all of the elements of the research plan have been completed.

Although the tasks have been organized into separate research components, a high level of integration and collaboration between the components is necessary to meet the objectives of plan. A summary of all tasks is provided in Table 7-1. Descriptions are provided in the sections that follow. 
Table 7-1 Research Plan - Summary of Tasks

\begin{tabular}{|c|c|c|}
\hline Phase & No. & Task \\
\hline \multirow[t]{3}{*}{ Archetype } & AR & Development of Archetype Buildings \\
\hline & AR.1 & Develop Archetype Building Configurations and Designs \\
\hline & AR.2 & Report on Archetype Buildings \\
\hline \multirow[t]{11}{*}{ Member } & ME & Investigation of Member Behavior \\
\hline & ME.1 & Conduct Computational Research \\
\hline & ME.1.1 & Develop Computational Models \\
\hline & ME.1.2 & Perform Parametric Analyses \\
\hline & ME.1.3 & Analyze Preliminary Test Specimens \\
\hline & ME.2 & Conduct Experimental Research \\
\hline & ME.2.1 & Finalize Test Specimens \\
\hline & ME.2.2 & Perform Member Tests \\
\hline & ME.2.3 & Evaluate Test Results \\
\hline & ME.3 & Perform Corroborating Computational Research \\
\hline & ME.4 & Report on Member Behavior and Preliminary Recommendations \\
\hline \multirow[t]{11}{*}{ Subassemblage } & SA & Investigation of Subassemblage Behavior \\
\hline & SA.1 & Conduct Computational Research \\
\hline & SA.1.1 & Perform Continuum Nonlinear Analysis of Subassemblages \\
\hline & SA.1.2 & Establish the Accuracy and Reliability of Panel Zone Predictions \\
\hline & SA.1.3 & Evaluate Fundamental Theories of Lateral Support \\
\hline & SA.2 & Conduct Experimental Research \\
\hline & SA.2.1 & Perform Initial Testing on Deep Column Subassemblages \\
\hline & SA.2.2 & Investigate Slab Effects, Lateral Support, and Selected Design Parameters \\
\hline & SA.2.3 & Investigate Additional Issues \\
\hline & SA.3 & Perform Corroborating Computational Research \\
\hline & SA.4 & Report on Subassemblage Behavior and Preliminary Recommendations \\
\hline \multirow[t]{9}{*}{ System } & SM & Investigation of System Behavior \\
\hline & SM.1 & Conduct Computational Research \\
\hline & SM.1.1 & Perform Preliminary Computational Studies \\
\hline & SM.1.2 & Perform Advanced Computational Studies \\
\hline & SM.2 & Conduct Experimental Research \\
\hline & SM.2.1 & Perform Hybrid Simulation Tests \\
\hline & SM.2.2 & Perform Earthquake Simulation Tests \\
\hline & SM. 3 & Perform Corroborating Computational Research \\
\hline & SM.4 & Report on System Behavior and Preliminary Recommendations \\
\hline \multirow[t]{4}{*}{ Products } & PR & Development of Final Products \\
\hline & PR.1 & Develop Improved Design and Assessment Recommendations \\
\hline & PR.2 & Develop Analytical Tools \\
\hline & PR.3 & Implementation in Codes and Standards \\
\hline
\end{tabular}




\subsection{Development of Archetype Buildings}

Development of archetype buildings (designated $A R$ ) is a Level 1 priority activity. Archetype building designs will be used to determine typical member sizes, subassemblages, and system configurations that are studied in the remaining elements of the research plan. Tasks for studying member behavior are described below.

Task AR.1: Develop Archetype Building Configurations and Designs. Archetype buildings are intended to be representative of the range of structural configurations that would be expected to use deep, slender wide flange columns. Development of archetype designs is described in Chapter 3. This task should occur during the first year of implementation.

Task AR.2: Report on Archetype Buildings. The resulting archetype buildings, configurations, designs, member sizes, and a discussion of preliminary key design variables will be summarized in an interim report upon completion of the work.

\subsection{Investigation of Member Behavior}

Investigation of member behavior (designated $M E$ ) is a Level 1 priority activity. Tasks for studying member behavior are described below.

Task ME.1: Conduct Computational Research. Member-based computational research is described in Chapter 4. This is the initial analytical work that will be used to establish the member-based experimental research program. Work will extend over a duration of approximately one and a half years (following the identification of archetype member sizes), and will consist of the following three subtasks:

- Task ME.1.1: Develop Computational Models. Computational modeling strategies are solidified and computational models are constructed for member analysis.

- Task ME.1.2: Perform Parametric Analyses. Parametric analyses are performed based on the guidelines presented in the plan, iteratively improving models and adjusting member size and properties as needed based on initial findings to ensure the proper range of behavior is covered in the parametric studies. Results are evaluated and synthesized.

- Task ME.1.3: Analyze Preliminary Test Specimens. A preliminary suite of experimental test specimens is developed, modeled, and analyzed to ensure a proper range of behavior is covered in the tests.

Task ME.2: Conduct Experimental Research. Member-based experimental research is described in Chapter 4. Experimental work will extend over a duration of approximately two years, some of which may be concurrent with the completion of computational research tasks. Work will consist of the following three subtasks: 
- Task ME.2.1: Finalize Test Specimens. The suite of test specimens is finalized based on the archetype buildings and results from the preliminary computational studies, considering the limits of available experimental testing equipment.

- Task ME.2.2: Perform Member Tests. Member test specimens are fabricated and tested.

- Task ME.2.3: Evaluate Test Results. Test results are evaluated and synthesized.

Task ME.3: Perform Corroborating Computational Research. Corroborating computational analyses will be conducted to refine the simulations conducted prior to testing and to calibrate with experimental results. Measured material and dimensional properties as well as final loading histories may be used. Work will extend over a duration of approximately two years, some of which may be concurrent with experimental testing.

Task ME.4: Report on Member Behavior and Preliminary Recommendations. Results from member-based computational and experimental research, and preliminary improved design recommendations, will be summarized in an interim report upon completion of the work.

\subsection{Investigation of Subassemblage Behavior}

Investigation of subassemblage behavior (designated $S A$ ) includes both Level 1 and Level 2 priority activities. Tasks for studying subassemblage behavior are described below. Lower priority tasks are identified as "Level 2."

Task SA.1: Conduct Computational Research. Subassemblage computational research is described in Chapter 5. Initial analytical work will be used to establish the subassemblage experimental research program, and ongoing analytical work will be used to perform corroborating studies. This work will extend over a duration of approximately two and a half years, and will consist of the following three subtasks:

- Task SA.1.1: Perform Continuum Nonlinear Analysis of Subassemblages. This task is divided into 3 parts extending over the duration of the subassemblage research program.

Part 1 consists of model development and calibration with past experimental research such as the SAC Joint Venture testing program. These analyses will allow gathering of additional information and understanding from prior test results.

Part 2 consists of a comprehensive parameter study that will be used to establish the subassemblage testing program.

Part 3 consists of ongoing analytical study in support of testing, and will continue over the duration of the experimental program. Each specimen that is tested will be analyzed to further evaluate the accuracy and reliability of models and to 
better estimate the effects of boundaries, lateral support, distribution of yielding and other factors.

- Task SA.1.2: Establish the Accuracy and Reliability of Panel Zone Predictions. This task consists of development of a comprehensive database and thorough review of past experimental data on panel zone response. This work will result in a thorough understanding of panel zone response and improved ability to simulate panel zone behavior for used in other subassemblage computational and experimental research tasks. This task is staged to begin shortly after Part 1 of Task SA.1.1, so that analytical results from past subassemblage research can be utilized, but this task should be completed relatively early in the overall program so that information can be used on other tasks.

- Task SA.1.3: Evaluate Fundamental Theories of Lateral Support. This task consists of performing a comprehensive review of past theoretical developments and available experimental data on lateral support and lateral-torsional buckling. The results of this task will provide recommendations to be used in the conduct of experimental investigations.

Task SA.2: Conduct Experimental Research. Subassemblage experimental research is described in Chapter 5. Experimental work could extend over a duration of approximately five years, and will consist of the following three subtasks:

- Task SA.2.1: Perform Initial Testing on Deep Column Subassemblages. This task consists of an initial test series intended to evaluate the effect of deep columns on moment frame behavior utilizing T-shaped subassemblages. This task is staged to begin after Part 1 of Task SA.1.1, with the actual testing performed in parallel with the remaining computational research studies. Results from this work will be used in later subassemblage experimental tasks.

- Task SA.2.2: Investigate Slab Effects, Lateral Support, and Selected Design Parameters. This task consists of a second series of more detailed testing intended to evaluate the effect of slabs, lateral support and restraint, and doubler and continuity plates on connection performance. This task will build upon computational research Tasks SA.1.1 (Parts 1 and 2), SA.1.2, SA.1.3, and experimental research Task SA.2.1.

- Task SA.2.3: Investigate Additional Issues. This task is a Level 2 priority activity that includes an allowance for performing an additional series of tests based on results derived from all prior research studies. It will likely be partially driven by results from member- and system-based research studies. As a result, this task is staged to occur later in the subassemblage research program.

Task SA.3: Perform Corroborating Computational Research. Corroborating computational analyses will be conducted in parallel with experimental research, under Part 3 of Task SA.1.1. Additional corroboration will be achieved through 
comparison and calibration with system-level computational and experimental results. Work will occur in parallel with the subassemblage experimental research tasks.

Task SA.4: Report on Subassemblage Behavior and Preliminary Recommendations. Results from subassemblage computational and experimental research, including preliminary improved design recommendations and practice-oriented analytical tools, will be summarized in an interim report upon completion of the work.

\subsection{Investigation of System Behavior}

Investigation of system behavior (designated $S M$ ) includes both Level 1 and Level 2 priority activities. Tasks for studying system behavior are described below. Lower priority tasks are identified as "Level 2."

Task SM.1: Conduct Computational Research. System-level computational research is described in Chapter 6. Work will extend over a duration of approximately seven years, beginning after the completion of the archetype building studies, and consisting of the following three subtasks:

- Task SM.1.1: Perform Preliminary Computational Studies. Preliminary computational studies consist of three parts and are expected to last two years.

Part 1 consists of the development of simplified models to investigate sensitivity to design variables, strong column weak beam ratios, P-Delta effects, ground motion input parameters, and dynamic instability for different ground motion sets. This information will used in Part 2.

Part 2 consists of the development of detailed building prototype models incorporating strength and stiffness deterioration and state-of-the-art component simulation models that have been calibrated with available experimental data. These models will be extensively analyzed using nonlinear response history analysis. This information will be used in Part 3.

Part 3 consists of studies on axial load ratios at end columns, loading histories, effects of cyclic deterioration on dynamic stability of moment resisting frames, assessment of slab effects, recommendations for SCWB ratios, panel zone design, and panel zone effect on seismic response of moment frame systems.

- Task SM.1.2: Perform Advanced Computational Studies. Advanced computational studies consist of three parts. They are staged to begin during the third year, and last for the duration of the research program.

Part 1 consists of re-design (if needed) of archetype buildings based on available design recommendations from member-based and subassemblage-based research. 
Part 2 consists of advanced component modeling (composite beams, panel zone, steel columns, base plates) and will explicitly address three-dimensional effects such as coupling of load ratios and biaxial bending particularly at end columns.

Part 3 is a Level 2 priority activity that includes advanced simulation models for other issues such as the effects of gravity framing, SCWB ratios, column splicing and modeling uncertainty on the seismic behavior of moment resisting frames. During the last two years of the system-based research program it is anticipated that advanced simulation models will be validated with available experimental data from system tests.

Task SM.2: Conduct Experimental Research. System-level experimental research is described in Chapter 6. Experimental work extends over a duration of approximately three and a half years, and will consist of the following two subtasks:

- Task SM.2.1: Perform Hybrid Simulation Tests. This task consists of hybrid simulation tests intended to quantify the axial restraint provided by a slab, evaluate stability of deep columns as part of a moment resisting frame, panel zone design and the effect of yielding on stability of deep beam-to-column connections, and issues related to SCWB ratios. It is expected to last about two years, and is staged to start after the completion of subassemblage tests and after the advanced computational studies are underway.

- Task SM.2.2: Perform Earthquake Simulation Tests. This task is a Level 2 priority activity. It includes a series of shake table tests on full-scale, full-frame specimens that is expected to last about two years. This work is intended to test the integrated results of all elements of the research plan in a full-scale system context.

Task SM.3: Perform Corroborating Computational Research. Corroborating computational research will be performed using updated analytical models from the advanced computational studies. Models will be updated based on information that becomes available from member, subassemblage, and system research.

Task SM.4: Report on System Behavior and Preliminary Recommendations. Systemlevel investigations are intended to confirm the results from all prior member and subassemblage research studies. Results from system computational and experimental research, and finalized improved design recommendations, will be summarized in an interim report upon completion of the work.

\subsection{Development of Final Products}

Preliminary recommendations for improved design provisions and practice-based analytical tools will be developed as part of each research component. These recommendations will be considered preliminary until confirmed by system-level computational and experimental investigations. Upon completion of the system-level 
studies, results for member-, subassemblage-, and system-based research will be synthesized in the final development stages. Tasks for development of final products are described below.

Task PR. 1: Develop Improved Design and Assessment Recommendations.

Preliminary recommendations developed under member- and subassemblage-based research will be confirmed in system-level investigations.

Task PR.2: Develop Analytical Tools. This task is a Level 2 priority activity. With sufficient data developed during member-, subassemblage-, and system-based research obtained during the member-based research studies, it is possible to develop new finite element formulations for deep columns. This task will have multiple stages, including element development, formulation calibration to experiments and refined analyses, and formulation validation to experiments and refined analyses.

Task PR.3: Implementation in Codes and Standards. Recommendations developed under this research plan are intended to be ready for direct integration into federally referenced guidelines, codes, and standards, including the NEHRP Recommended Provisions for Seismic Regulations for New Buildings and Other Structures (FEMA, 2009c). They are also intended to be directly useful to standards development committees such as those responsible for developing ANSI/AISC 360, Specification for Structural Steel Buildings, ASCE/SEI 7, Minimum Design Loads for Buildings and Other Structures, ASCE/SEI 41, Seismic Rehabilitation of Existing Buildings.

A small working group consisting of principal investigators from selected analytical and experimental projects in the research plan can be assigned to work with members of AISC Task Committee 9 on seismic design to generate proposed modifications to the ANSI/AISC 341, Seismic Provisions for Structural Steel Buildings, and ANSI/AISC 358, Prequalified Connections for Special and Intermediate Steel Moment Frames for Seismic Applications.

\subsection{Recommended Schedule}

A possible schedule for the work is shown in Figure 7-1. Based on the scope of the overall program, the estimated overall project schedule is approximately eight years. Due to complex interrelationships between the tasks and uncertainty in the procurement of contracted experimental research, this schedule must be considered approximate. 


\begin{tabular}{|c|c|c|c|c|c|c|c|c|}
\hline Phase & Year 1 & Year 2 & Year 3 & Year 4 & Year 5 & Year 6 & Year 7 & Year 8 \\
\hline Archetype Developmer & $\Rightarrow$ & & & & & & & \\
\hline Member Investigations & & & & & & & & \\
\hline Subassemblage Invest & & & & & & & & \\
\hline System Investigations & & & & & & & & \\
\hline Product Development & & & & & & & & \\
\hline
\end{tabular}

Figure 7-1 Approximate overall schedule for the recommended research plan.

Alternative schedules considering more concurrent or more sequential tasks are certainly possible. Although the major research components can be considered separately, there is expected to be a significant level of interaction and coordination required between many of the tasks to achieve the intended results. Interactions between subtasks are illustrated in Figure 7-2.

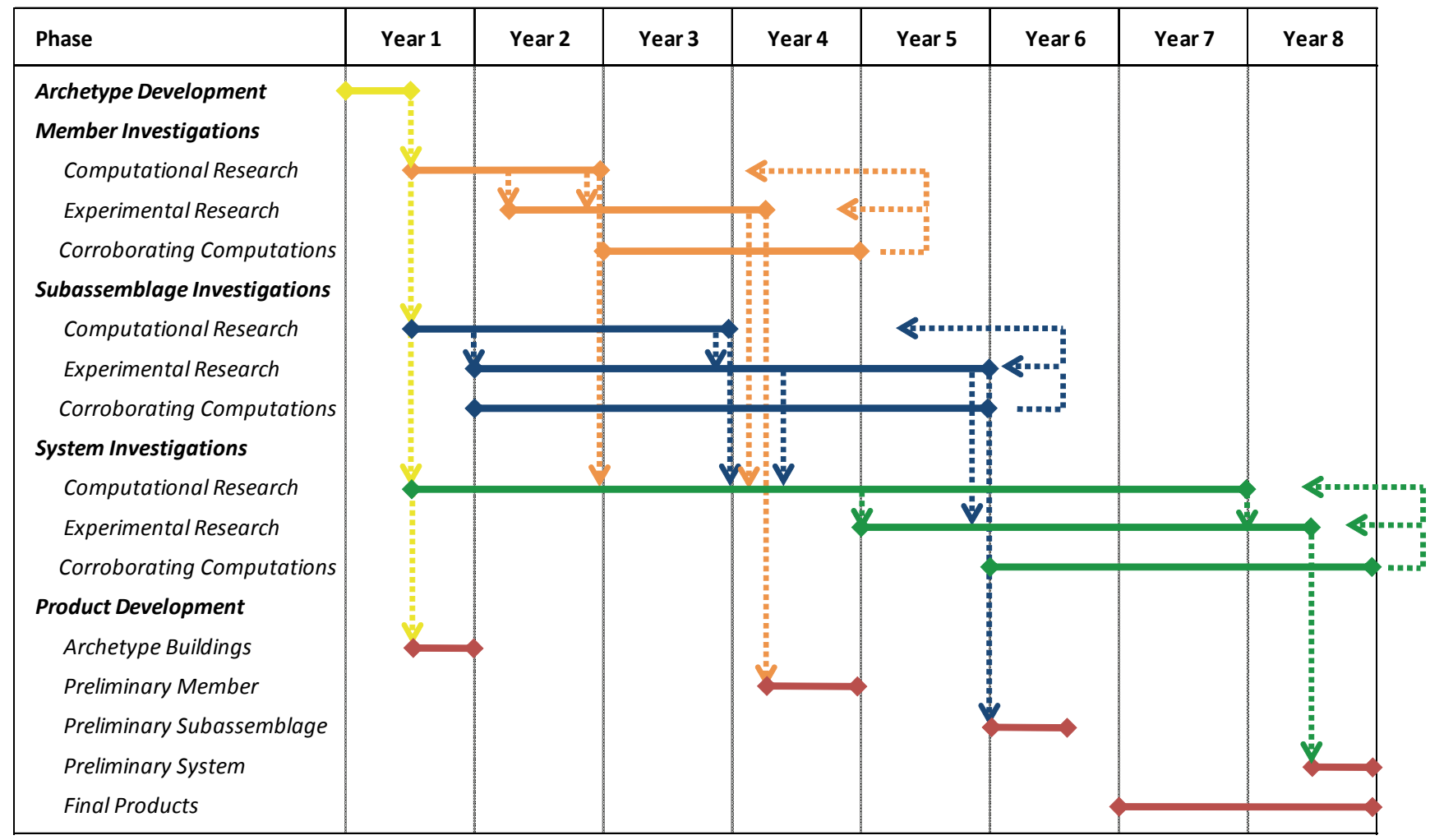

Figure 7-2 Approximate detailed schedule showing subtasks and interrelationships between major elements of the recommended research plan.

\subsection{Estimated Budget}

It is anticipated that NIST will conduct some elements of the research program internally, notably the analytical work. Portions of the analytical work, as well as the experimental work, may well be competitively awarded to outside contractors to perform, contingent on the availability of funds. Due to the competitive nature of the anticipated procurement process, potential variations in material and fabrication costs, and uncertainty in the current economic environment, establishing a precise 
estimate for each element of the research plan was not feasible. Based on historical expenditures for research on structural steel programs funded by the Federal government in the past, it is estimated that implementation of this program will require up to $\$ 10 \mathrm{M}$, excluding system-based shake table testing, which has been identified as a Level 2 priority. An additional $\$ 2 \mathrm{M}$ is estimated to conduct the recommended shake table testing on full-scale, full-frame systems under the systembased research plan.

It is anticipated that NIST will conduct cost estimating exercises as part of the procurement process to confirm and update the above estimates as the program is implemented.

\subsection{Key Collaborators}

Although NIST will manage the overall program and perform selected portions of the work, the objectives of the research program would benefit from support, interaction, and coordination with other agencies of the Federal government, representative industry organizations, and codes and standards development organizations including:

- National Science Foundation (NSF)

- Federal Emergency Management Agency (FEMA)

- American Institute of Steel Construction (AISC)

- American Iron and Steel Institute (AISI)

- American Society of Civil Engineers (ASCE)

Opportunities to jointly fund research activities, or collaborate with these organizations, should be pursued. 


\section{Appendix A}

\section{Literature Review}

This appendix presents the results of an extensive literature review focusing on past integrated experimental and analytical research that has been conducted on steel beams, beam-columns, beam-to-column connections, and moment resisting frame system performance under static and cyclic loading. The focus is on wide flange columns, but information on other shapes (i.e., steel tubular columns) is also presented.

\section{A.1 Centrally Loaded Columns}

A comprehensive list of reference material related to centrally loaded columns can be found in Driver et al. (2003) and the sixth edition of the Guide to Stability Design Criteria for Metal Structures (Zeimian, 2010). It is well known that a perfectly straight, prismatic column, with concentric loading and well-defined restraints, buckles elastically in a flexural mode at a strength equal to the Euler load, $P_{E}$. Many columns, however, are in a range of slenderness where they are no longer elastic when local buckling takes place (i.e., stiffness degradation occurs). Shanley (1947) contributed to improved understanding of the post-buckling behavior of inelastic columns. Research studies on the design of steel columns (Robertson, 1925; Bjorhovde and Tall, 1971; Bjorhovde, 1972; Hall, 1981; and Fukumoto et al., 1983) document over 1000 centrally loaded column tests reported in the literature for different column types. In these same studies, design formulas for steel columns are included that are either: (1) based on empirical results from column tests; or (2) are based on a yield limit state, tangent modulus theory, or maximum strength (see Zeimian, 2010).

Earlier studies on steel columns focused on the effect of residual stresses in hot-rolled shapes (Beedle and Tall, 1960). The magnitude and distribution of residual stresses in hot-rolled shapes depend on the type of cross section, rolling temperature, cooling conditions, straightening procedures, and the material properties of the steel. Tall (1964) showed that for heavier shapes, residual stresses vary significantly through the thickness. Examples can be found in Brozzetti et al. (1970a, 1970b). Tall (1964) concluded that the effect of steel strength on the residual stress distribution is not as great as the effect of geometry, and residual stresses in the flanges have the most significant effect on the strength of wide flange columns.

Systematic examination of the separate and combined effects of residual stresses and initial out-of-straightness on column strength was performed at the University of 
Michigan (Batterman and Johnston, 1967) and Lehigh University (Bjorhovde, 1972). The failure mode in these studies was flexural buckling about the weak axis. The main findings concluded that residual stresses had little effect on the maximum strength of slender columns, either straight or initially crooked, and that such members have a strength approaching the Euler load. Differences in column strength caused by variations in the shape of the residual stress pattern are smaller for initially crooked columns than for initially straight columns. This is a result of the early flexural behavior of initially curved members. Initial out-of-straightness is normally expressed as a fraction of the length, $L$, of the member (about L/1000). Dux and Kitipornchai (1981) and Essa and Kennedy (1993) demonstrated that most hot-rolled wide flange sections tend to have initial out-of-straightness close to maximum permissible tolerances. Information on welded build-up shapes can be found in Alpsten and Tall, 1970; McFalls and Tall, 1970; Alpsten, 1972; Brozzetti et al., 1970b; and Bjorhovde et al., 1972) but is not discussed here.

Extensive studies on the influence of end restraint on the strength and behavior of steel columns have been conducted by Chen (1980), Jones et al. (1980, 1982), Razzaq and Chang (1981), Chapuis and Galambos (1982), Vinnakota (1982, 1983, 1984), and Shen and $\mathrm{Lu}$ (1983). These studies have focused on developing methods of accounting for connection flexibility in providing effective end restraint for framed columns. An extensive review of research on the behavior and modeling of column connections is provided in Chen (1987, 1988), Chen and Lui (1991), Beedle (1993), and Chen et al. (1996). Based on an evaluation of alternative connection models available in the literature, a three-parameter connection power function model proposed by Kishi and Chen (1990), together with its large database (Kishi and Chen, 1986; Chen and Kishi, 1989) and design aids (Kishi et al., 1993), can be recommended for general use.

Since its introduction in 1961, the effective length factor, $K$, has been widely accepted and used for assessment of column stability in various structure types. Despite widespread acceptance, however, the $K$-factor approach involves a number of major assumptions. Refinements for effective length computations continue to be studied by researchers (Hellesland and Bjorhovde, 1996a; Hellesland and Bjorhovde,1996b). The direct analysis method (Surovek et al., 2005) that is presented in ANSI/AISC 360-10, Specification for Structural Steel Buildings (AISC, 2010c), is the preferred approach.

More recently, Lamarche and Tremblay (2011) conducted a number of tests on seismically induced cyclic buckling of steel columns in steel braced frames, with a focus on weak axis buckling. They used four nominally identical W12×87 (Class 1) columns made of A992 steel with an effective slenderness ratio of 48 . The effects of residual stress and strain-rate on column performance were taken into account. Test protocols included monotonic and cyclic, concentric and eccentric axial loading. Studies showed that steel columns could sustain several cycles of inelastic buckling 
under seismic loading while maintaining sufficient compressive capacity to support applied gravity loads. Residual stresses affected the column response at the first occurrence of buckling, also causing a gradual reduction in tangent stiffness prior to buckling and a reduction in compressive resistance. High strain rates anticipated during strong earthquake shaking increased column buckling and post-buckling strengths. Using the OpenSEES, Open System for Earthquake Engineering Simulation, analysis platform (McKenna 1997), Lamarche and Tremblay (2011) concluded that the cyclic buckling response of steel columns is predicted adequately when using nonlinear beam-column elements and cross-section fiber discretization, provided that residual stresses and strain rate effects are modeled.

\section{A.2 Research on Beam-Columns}

Beam-columns are defined as members subjected to a combination of axial force and bending moment. Bending moments in beam-column members stem from transverse loading acting between the member ends, from loading on adjacent members in rigidly framed structures, or from the eccentricity of reactions and nominal axial forces in simply framed structures. When addressing the behavior of beam-columns in rigidly framed structures, it is usually necessary to consider the influence of the surrounding structure, which leads to the study of subassemblies and complete frames. Based on Zeimian (2010), for non-sway beam-columns (non-seismic) with doubly symmetric cross sections, three classes of problems can be identified:

1. Members subjected to major axis bending and braced against minor axis flexure (or subjected to minor axis bending), which will collapse by excessive in-plane bending deflections. This case corresponds to the interaction between column flexural buckling and simple uniaxial beam bending.

2. Unbraced members subjected to major axis bending, which will collapse by an interaction between column flexural buckling, beam lateral-torsional buckling, and uniaxial beam bending.

3. Unbraced members subjected to biaxial bending, which will collapse by an interaction between column flexural buckling, beam lateral-torsional buckling, and biaxial beam bending. This case constitutes the most general case.

\section{A.2.1 Uniaxial Bending: In-Plane Strength}

For slender beam-columns, the combined action of the axial load, $P$, and the deflections produced by the primary (first-order) moments, $M_{o}$, leads to further deflections and the so-called secondary moments (not to be confused with secondorder moments). In the particular case of simply supported beam-columns subjected to end moments causing double-curvature bending, the maximum second-order moment may occur at the end(s) of the member (even for high axial force values), thus being equal to the maximum first-order moment. For members subjected to 
either: (1) major axis bending and braced against minor axis flexure; or (2) minor axis bending, a conventional form used as a starting point for several design formulas was first introduced by Johnston (1976):

$$
\frac{P}{P_{u}}+\frac{M_{0}}{M_{u}\left(1-P / P_{e}\right)} \leq 1
$$

where $P_{u}$ and $M_{u}$ are the axial and bending strength under only one internal force or moment, and $P_{e}$ is the elastic critical load for buckling in the plane of the applied moments. The term in the parentheses may be considered an amplification factor on the first order moment for the purpose of obtaining the second-order moment, which is based on a numerical solution of Galambos and Ketter (1961). Additional analytical solutions for wide flange beam-columns have been derived by a number of researchers (Ketter, 1962; Lu and Kamalvand, 1968; Chen, 1970; Chen, 1971a; Chen, 1971b; Yu and Tall, 1971; Young, 1973; Chen and Atsuta, 1972; Ballio et al., 1973; Ballio and Campanini, 1981; and Lu et al., 1983). These formulations provide an acceptable fit for all wide flange sections, including those fabricated by welding, and have been verified with a number of experimental studies with hot-rolled shapes (Johnston and Cheney, 1942; Ketter et al., 1952; Ketter et al., 1955; Galambos and Ketter, 1961; Yu and Tall, 1971; Mason et al., 1958; van Kuren and Galambos, 1964; Dwyer and Galambos, 1965; Bijlaard et al., 1955; and Lay and Galambos, 1965).

For beams-columns subjected to unequal end moments or transverse loads, Equation A-1 is slightly modified to:

$$
\frac{P}{P_{u}}+\frac{C_{m} M_{0}}{M_{u}\left(1-P / P_{e}\right)} \leq 1
$$

In this equation $C_{m}$ is an adjustment factor and $M_{o}$ is taken as the maximum first-order moment. Equation A-2 represents the condition of failure stemming from instability due to excessive bending occurring within the member length. This implies that, in compact sections, end moments must be limited by the plastic hinge moment modified to account for the effect of axial compression. AISC provisions include expressions for $M_{p c}$ that are applicable to wide flange shapes. These curves have been verified with experimental data summarized in a number of studies. For additional consideration of end moments, Pillai (1974) suggested an equation containing the interaction between column plastic hinging and axial force. Kemp (1984), based on an earlier development by Lay (1974), proposed an equation for limiting the slenderness ratio of wide flange sections. Based on this work, van Manen (1982) published a more general study on the interaction between axial force, end moment, slenderness, and rotation capacity. 


\section{A.2.2 Uniaxial Bending: Lateral-Torsional Buckling}

When a wide flange section is bent about its major axis, there is a tendency for it to fail by deflecting sideways and twisting. When such a member is a beam-column, the presence of an axial compressive force will accentuate this tendency because the predominant mode of failure under pure compression is buckling about the minor axis. Beam-columns typically bend about the major axis and therefore exhibit interaction between column and beam buckling. In this case, Equations A-1 and A-2 are converted to:

$$
\begin{aligned}
& \frac{P}{P_{e y}}+\frac{M_{0}}{M_{E}\left(1-P / P_{e x}\right)} \leq 1 \\
& \frac{P}{P_{e y}}+\frac{C_{m} M_{0}}{M_{E}\left(1-P / P_{e x}\right)} \leq 1
\end{aligned}
$$

where $P_{e x}$ and $P_{e y}$ are the elastic critical loads about major and minor axis flexural buckling, and $M_{E}$ is the beam elastic critical moment for lateral-torsional buckling. In Equations A-3 and A-4, it is also necessary to check the resistance of a beam-column cross section strength at the member ends in order to identify if elastic or inelastic lateral-torsional buckling will occur. A number of researchers have investigated this issue (Vinnakota, 1977; Fukumoto and Galambos, 1966; Galambos et al., 1965; Miranda and Ojalvo, 1965; Bradford and Trahair, 1985, 1986; Pi and Trahair, 1992a, 1992b, 1994a, and 1994b). A summary of all available work on this subject can be found in Trahair (1993). A number of experimental studies have been conducted to investigate the effect of lateral-torsional buckling (van Kuren and Galambos, 1964). In some of those studies lateral-torsional buckling occurred because of inadequate bracing.

\section{A.2.3 Biaxial Bending of Slender Beam-Columns}

A number of investigators (Birnstiel, 1968; Chubkin, 1959; and Anslijn, 1983) have conducted experimental research on beam-columns subjected to biaxial bending with a focus on wide flange sections with slenderness ratios, $L / r_{y}$, ranging from 40 to 150 . Zeimian (2010) includes a comprehensive list of reports on this topic. These results, together with analytical derivations (Djalaly, 1975; Lindner, 1972; Vinnakota, 1977; Rajasekaren, 1977; Duan and Chen, 1990) have led to design procedures for biaxial bending. Equation A-4 can be generalized for biaxial bending as follows:

$$
\frac{P}{P_{u}}+\frac{C_{m x} M_{x}}{M_{u x}\left(1-P / P_{e x}\right)}+\frac{C_{m y} M_{y}}{M_{u y}\left(1-P / P_{e y}\right)} \leq 1
$$

where $P_{u}$ is the ultimate strength of an axially loaded column, and $M_{u x}$ and $M_{u y}$ are the ultimate bending moments of beams bent about the major (x) and minor (y) axes, respectively. However, it should be noted that Equation A-5 is often a rather poor 
predictor of capacity. In compact wide flange beam-columns, $M_{u y}$ would be the minor axis full plastic moment capacity and $M_{u x}$ would be reduced due to the possibility of lateral-torsional buckling. Theoretical procedures for assessing beam-column strength under biaxial loading are rather complex, even within the elastic range, so numerical solutions are required (Culver, 1966a, 1966b). Many hot-rolled or welded beamcolumns may also be susceptible to the interaction between local and overall buckling.

\section{A.2.4 Behavior of Beams-Columns under Reversed Cyclic Loading}

Nakashima et al. (1990) tested wide flange beam-columns subjected to axial load and unidirectional bending. Strong axis and weak axis rotation and torsion were prevented at both ends of the specimens. For each test, an axial load was imposed to a specific level, with axial loads varying from 0.0 to 0.6 times the axial capacity. Lateral deflections were imposed on all specimens. Since all test specimens had compact sections, local buckling did not occur at early stages of loading. Because of small width-to-thickness and slenderness ratios chosen, effects of local buckling and lateraltorsional buckling on the in-plane characteristics of the specimens tested were found to be negligible.

The strength and ductility of the specimens were compared to strength and ductility provisions contained in the AISC Load and Resistance Factor Design Specification for Structural Steel Buildings (AISC, 1986). In this study, the ductility ratio was defined as the ratio between the deflection, $\Delta_{c}$, corresponding to $0.95 M_{b}\left(M_{b}\right.$ is the first-order maximum end moment) divided by the yield lateral deflection, $\Delta_{y}$. The experimental strength was never lower than the design strength, and was higher by $11 \%$ on average. The maximum unbraced length stipulated in the $L R F D$ Specification, intended as a condition to achieve ductility ratios of 4, was found to achieve only small ductility ratios (not more than 2.0 ).

Nakashima (1994) performed an investigation on the variation of plastic deformation capacity of steel beam-columns subjected to axial load and uniaxial bending based on available experimental data from Japanese researchers (Nakashima et al., 1990). This investigation revealed that neither residual stresses nor initial out-of-straightness was a major factor affecting the ductility ratio, but that strain hardening is very influential, suggesting that strain hardening is a major cause of large variations observed in experimental ductility ratios.

More recently, Lignos and Krawinkler (2009) gathered experimental data on 115 tubular steel beam-columns subjected to combined axial load and bending. These tests were conducted mostly by Japanese researchers (Tsuda and Matsui, 1998; Tsuji and Nakatsura, 1986; Kurata et al., 2005; Kawaguchi and Morino, 2001; Yamanaka and Yamada, 1984; and Sugiyama and Igarashi, 1986). Through multivariate regression analysis, trends for deterioration parameters were established for tubular steel beam-columns with respect to slenderness ratio and axial load ratio. The main conclusion is that tubular steel beam-columns deteriorate rapidly for depth $(D)$ to 
thickness $(t)$ ratios $D / t>30$ and high axial load ratios $N / N_{y}>0.3$. This was confirmed by a full-scale collapse test of a 4-story steel structure conducted at the E-Defense facility in Japan (Suita et al., 2008). The structure collapsed in a first story mechanism due to column bending strength deterioration caused by local buckling. The tubular steel columns in the test structure had $D / t$ ratios equal to 30 .

Nakashima et al. (2002) conducted an analytical study of the lateral-torsional instability and lateral bracing effects of wide-flange steel beams subjected to cyclic loading. Numerical analyses using the large deformation theory were conducted to collect the necessary data. Wide-flange steel beams were examined in double curvature and subjected to cyclic loading with increasing amplitudes up to a maximum end rotation of 0.045 radians. Cross-sectional properties, slenderness ratios, material strength, loading history, and unbraced lengths were chosen as analytical variables. For slenderness ratios about the weak axis greater than 100, the strength that can be sustained under cyclic loading was much smaller than the strength obtained under monotonic loading due to accumulation of out-of-plane deformations. Based on equations proposed for the beam unbraced length, no detrimental reduction in strength is present in cyclic loading up to the maximum beam end rotation of 0.045 radians. It was also found that AISC unbraced length requirements at the time were a reasonably conservative measure to ensure sufficient beam rotation capacity.

Nakashima et al. (2002) also analyzed the lateral instability of reduced beam section (RBS) beams. It is notable that an RBS beam is not necessarily more susceptible to lateral instability than a corresponding standard beam. This is primarily because of a smaller yielding region and smaller forces induced in the cross section of the RBS beam. Lateral bracing requirements stipulated for standard beams are applicable for ensuring sufficient rotation capacity for RBS beams if local buckling effects do not occur. Lignos and Krawinkler (2011) developed multivariate regression equations for predicting the plastic rotation capacity and post-buckling rotation capacity of RBS beams. Their assessment was based on a data set of 107 RBS beams ranging in size from W21 to W36. They concluded that as long as the ratio between the distance from the column face to the nearest lateral brace divided by the radius of gyration about the weak axis of the beam, $L_{b} / r_{y}$, is close to or smaller than $2500 / F_{y}$, as required in ANSI/AISC 341-10, Seismic Provisions for Structural Steel Buildings (AISC, 2010a), then the plastic rotation capacity of RBS beams is not greatly affected by $L_{b} / r_{y}$.

Astaneh and Ravat (1998) conducted a testing program with five cyclic tests of vertical HP14 $\times 89$ pile specimens $\left(b_{f} / 2 t_{f}=11.9, d / t_{w}=11.5\right)$ fabricated with A36 steel and constructed with reinforced concrete pile caps. Three specimens were tested under combined axial load and bending, and results from these tests are applicable to steel columns subjected to the same loading conditions. The first specimen, loaded in strong-axis bending, failed in flexure below the pile cap. Flange local buckling occurred at a drift ratio of about 0.045 radians, indicating relatively good 
performance, primarily due to the small $d / t_{w}$ ratio of the section. The second specimen, tested along a 45-degree axis, failed in combined flexural and local buckling. Flange local bucking occurred at a distance away from the pile cap (3.5 feet) with no indication of web buckling at a drift ratio of about 0.035 radians. Severe deterioration due to combined local buckling occurred at a drift ratio of about 0.066 radians. The third specimen, loaded in weak-axis bending, deteriorated rapidly due to local buckling of both flanges at a story drift ratio of about 0.05 radians.

In New Zealand, MacRae et al. (1990) tested a number of cantilever steel columns (250UC73) made of Grade 250 steel. These columns were designed as beam-column members in moment resisting frames. They were subjected to combined axial loads and reversed cyclic lateral deformations. Axial shortening exceeding $7 \%$ of the column length was reported in the columns. Axial shortening increased as local buckling became more significant during the loading history. This same study showed that axial shortening is independent of the plastic hinge length, $L_{p}$. A number of additional experimental and analytical studies related to column behavior under combined axial load and bending have been conducted in New Zealand (e.g., McRae et al., 2006; Peng et al., 2007, 2008; and NZSEE 2006).

Newell and Uang (2006) conducted cyclic static tests on nine full-scale heavy W14 columns with cross sections complying with ANSI/AISC 341 seismic compactness requirements. The columns were fixed at each end and subjected to lateral displacement histories while carrying axial loads of $0.35 P_{y}, 0.55 P_{y}$, and $0.75 P_{y}$. The loading protocol was obtained through rainflow counting of story drift histories based on response history analysis of 3-story and 7-story buckling restrained braced frame structures. The specimens sustained story drifts of 0.07 to 0.09 radians before a $10 \%$ reduction in maximum moment was observed. Examples of the hysteretic response of $\mathrm{W} 14 \times 132$ and $\mathrm{W} 14 \times 176$ columns at $P / P_{y}=0.75$ are shown in Figure A-1.

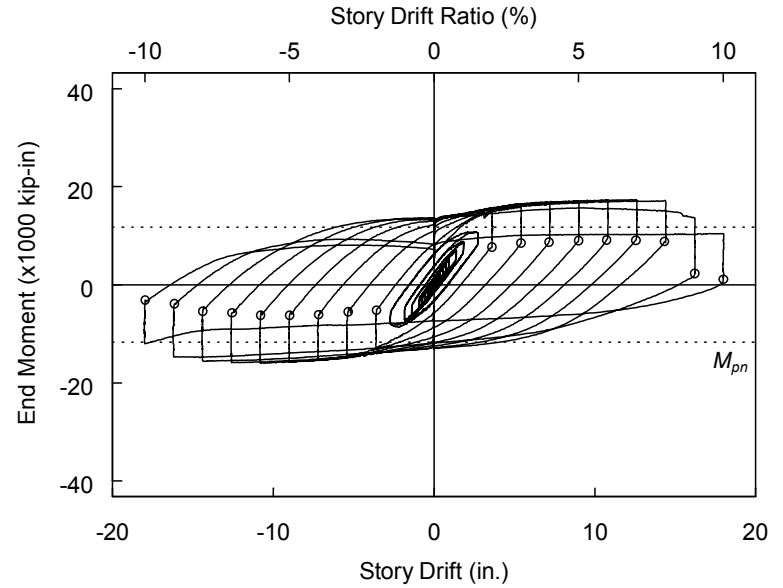

(a) $\mathrm{W} 14 \times 132, P / P_{y}=0.75$

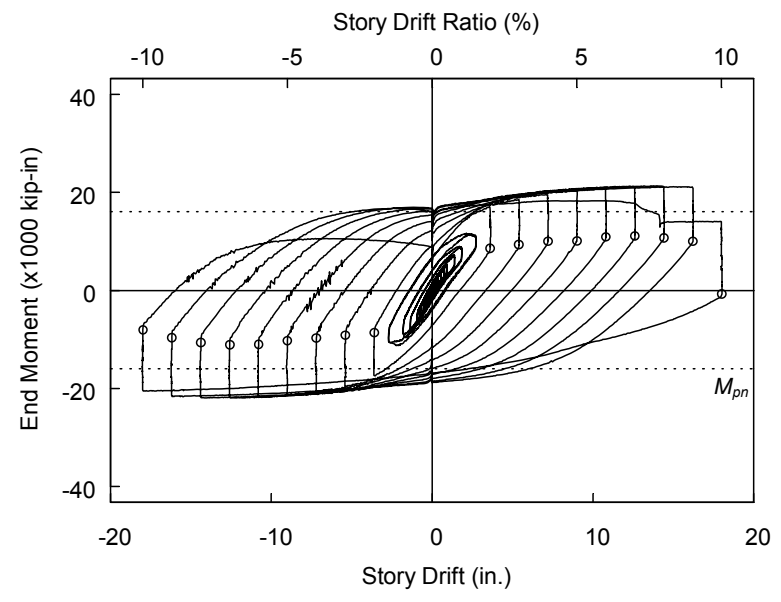

(b) W14x176, $P / P_{y}=0.75$

Figure A-1 Hysteretic response of two fixed-ended heavy columns subjected to combined axial load and bending (data from Newell and Uang, 2006). 
Because the specimens had stocky webs, flange local buckling dominated the buckling response in all tests. For the lightest column supporting $0.75 P_{y}$, the $b_{f} / 2 t_{f}$ and $h / t_{w}$ ratios corresponded to 1.0 and 0.43 times ANSI/AISC 341 seismic compactness limits.

In the study by Newell and Uang (2006), detailed finite element analyses were carried out. Simulated results matched experimental results and it was determined that a typical initial residual stress distributions did not significantly affect the P-M interaction or moment versus drift response of the specimens. However, the cyclic behavior of deep W27 columns, as predicted by analysis, indicated that significant strength degradation occurs in these specimens due to severe flange and web local bucking. Figure A- 2 shows the simulated hysteretic behavior of a W27× 146 column under different axial load ratios. Note the rapid loss of strength in the negative loading direction, even for axial load ratios equal to 0.35 . These findings are consistent with findings from a steel database for deterioration modeling of steel components (Lignos and Krawinkler, 2011) for slender wide flange sections. The precapping rotation capacity of these members ranges from about 1.5 radians to about 1.8 radians, and their post-capping rotation capacity (after first occurrence of local buckling) is not more than 0.15 radians.

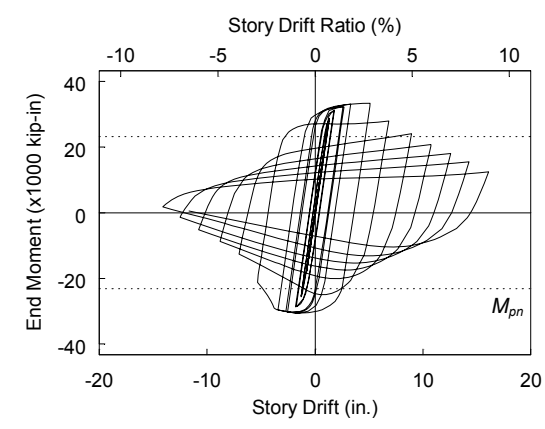

(a) $35 \% P_{y}$

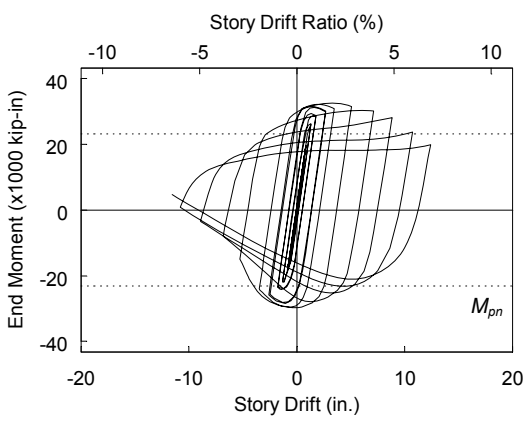

(b) $55 \% P_{y}$

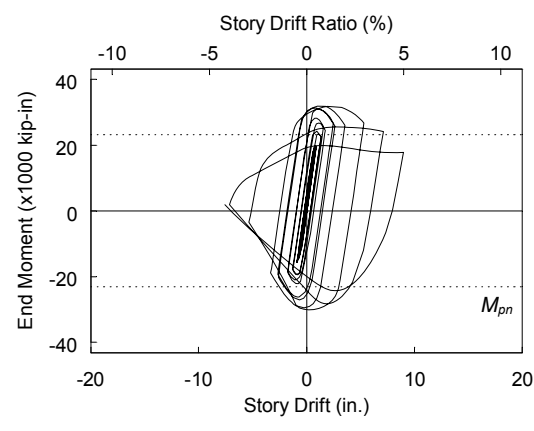

(c) $75 \% P_{y}$

Figure A-2 Cyclic response of a W27×146 column under combined axial load and bending (simulated results from Newell and Uang, 2006).

\section{A.3 Research on Beam-to-Column Connections}

This section summarizes past research related to behavior and performance of beamto-column connections including boundary conditions, column and panel zone behavior, strong column-weak-beam ratio, lateral bracing, slab effects, and loading history.

\section{A.3.1 Boundary Conditions}

Connection studies in the early 1970s used a cantilever beam test arrangement (Popov and Pinkney, 1969; Popov and Stephen, 1970). Later studies (Krawinkler et al., 1971; Bertero et al., 1973) examined the behavior of beam-to-column subassemblages that simulate overall frame behavior rather than simply considering connection behavior. 
These studies illustrated the effect of panel zone yielding on the overall performance of a beam-to-column connection. The SAC Joint Venture, which was formed in 1994 as a joint venture between the Structural Engineers Association of California, the Applied Technology Council, and the Consortium of Universities for Research in Earthquake Engineering, embarked on a problem-focused research program to develop interim solutions for the identification, evaluation, repair and modification of welded steel moment frame buildings damaged in the Northridge earthquake. During the SAC Phase I and II testing programs (FEMA, 2000c; Lignos et al., 2010) the main beam-to-column connection assemblies used for experimental testing were the Tshaped and cruciform test subassemblages. These subassemblages are based on the assumption that the inflection points in beams and columns in a steel frame structure are located at mid-length (or mid-height) during the entire response history. The notion of "fixed" points of inflection has since been shown to be an oversimplification. In addition, tests that were conducted as part of the US-Japan cooperative program (Leon and White, 1998) and others that evaluated overall system behavior rather than beam-to-column connection behavior only (Cordova and Deierlein, 2005; Nakashima et al., 2006) demonstrated that the axial restraint that can be provided to the steel beam by the slab is underestimated by T-shaped and cruciform subassemblages.

Few tests have been conducted in a cruciform configuration with the presence of axial load on the columns. Hajjar et al. (1997) investigated the effect of composite concrete floor slabs on the behavior of fully-restrained moment connections that failed during the 1994 Northridge earthquake. In these tests a constant axial load was applied, however, stocky columns (W14×211) were used, and beam sizes were W27×94. Ricles et al. (2004b) conducted an experimental study involving ten full-scale moment resisting connections with square concrete-filled steel tube columns and wide flange steel girders (W24×62). A constant axial compression load was applied to the columns. Results indicated that moment resisting connection details can be economically designed to achieve more than 0.045 radians of inelastic story drift under cyclic loading. Such details include split-tee connections without a shear tab, weak panel zone connections, and extended tee connections. The use of interior diaphragms in a tube column was shown to locally stiffen the joint, but also leads to strain concentrations and fracture of the beam flanges at weld access holes.

\section{A.3.2 Column Behavior}

Popov et al. (1975) conducted cyclic tests on interior cruciform beam-column subassemblies with wide flange columns. Axial load ratios in the columns and the direction of bending were varied in the tests. Column cross sections met current ANSI/AISC 341-10 seismic compactness requirements with $b_{f} / 2 t_{f}$ and $h / t_{w}$ ratios well below the respective limits. Specimens were designed using a strong-beam design approach to trigger yielding in the columns. In the tests, plastic rotations developed 
unevenly in the top and bottom column segments, indicating the propensity for a story mechanism to form in systems with weak columns. The phenomenon was more pronounced in columns supporting the highest axial loads $\left(0.6 P_{y}\right.$ and $\left.0.8 P_{y}\right)$. The contribution of the column deformation to total horizontal displacement was also more important for such columns, with individual column segments experiencing drifts of 0.06 to 0.08 radians, but the response of these specimens was characterized by a sharp drop in strength resulting from flange buckling.

Conversely, columns supporting an axial load of $0.30 P_{y}$ could sustain cyclic flexural demands about minor and major axes corresponding to a story drift ratio of about 0.03 radians without significant strength degradation (less than 10\%). Similar behavior was observed in tests with axial load ratios of $P / P_{y}=0.5$, except that the drop in lateral resistance was more severe when the columns were bent about their minor axes. A strain-hardening response was observed in all columns, which had a positive impact on column flexural resistance. It was found that inelastic rotations should be avoided in columns with $P / P_{y}>0.5$, and that moderate inelastic demand is permissible if $P / P_{y}<0.5$.

When used with RBS connections, deep narrow columns can also be subjected to twisting moments due to eccentric compression forces from the beam bottom flange upon lateral-torsional buckling of the beams. Based on tests conducted on RBS specimens without a concrete floor slab, Uang et al. (2000) proposed a procedure to verify column stresses at large story drifts, including torsional effects, and determine if additional beam bracing is needed. An analytical study by Shen et al. (2002), and analysis and experimental work by Ricles et al. (2004a), indicated that twisting of deep columns in RBS connections can be reduced with the presence of a floor slab and a supplemental brace located at the end of the RBS segment. Based on the same study, Ricles et al. (2004a) provided guidelines to determine the column torque at $4 \%$ story drift for cases with and without a supplemental lateral brace. Cruciform beamto-column subassemblies were tested with $\mathrm{W} 30 \times 108$ and $\mathrm{W} 36 \times 150$ beams with RBS connections and a range of deep columns (W24×131 to W36 $\times 230$ ). It should be pointed out that there were no plastic hinges in these columns. The study concluded that the fracture potential and column twist in an RBS connection depends on the section modulus and torsional rigidity of the column section, where larger stresses in the column flange can lead to a potential for higher ductile fracture in the connection along with twisting of the column. If the stresses in the column flanges are lower, an RBS connection with a deeper column can have a smaller ductile fracture potential than an RBS connection with a shallower column.

Based on combined finite element and experimental studies conducted by Ricles et al. (2004a), the ratio $h / t_{c f}^{3}$ or column depth, $d_{c}$, alone, is not a clear indicator of the effects of an RBS connection on a deep column. Consideration must be given to the torsional and flexural properties of the column section and the size of the beam in the 
beam-to-column connection. Larger beams will result in a greater stresses applied to the column.

RBS connections to deep columns appear to perform better than welded unreinforced flange-welded (WUF-W) web connections to deep columns. Finite element parametric studies by Ricles et al. (2004b) show that WUF-W connections develop a larger plastic strains, resulting in greater potential for ductile fracture than RBS connections. Because of this, test results based on RBS connections to deep columns cannot be extrapolated to WUF-W connections to deep columns.

Similar findings have been reported in FEMA 355D, State of the Art Report on Connection Performance (FEMA, 2000c), after evaluation of a number of experimental results that were conducted on various post-Northridge connections with deep columns ranging from W24×279 to W27×258 (Ricles et al., 2000; Whittaker and Gillani, 1996; and Gilton et al., 2000). It should be noted that in these subassemblages there were no plastic hinges. From these studies, it was concluded that connections with deep columns will provide less desirable seismic performance than comparable strong axis connections with W12 and W14 column sections. Also, deep column connections should always be designed with continuity plates, which can help reduce local demands on the $k$ region, and enhance lateral stability of the deep column and the connection.

Alternatively, concrete-encased or concrete-filled tube composite columns can be used to enhance column local buckling behavior and flexural ductility (e.g., Ricles and Paboojian, 1994; Ricles et al., 2000; Hajjar and Gourley, 1996; Tort and Hajjar, 2007; El-Tawil and Deierlein, 1999; and Varma et al., 2002, 2004). These columns also possess higher flexural stiffness (for drift control) and higher torsional stiffness (i.e., flexural-torsional buckling need not be checked). Gourley et al. (1995) compiled a database of experimental research conducted on concrete-filled tube (CFT) members through 1995. This information was utilized by Hajjar et al. (1997) and Hajjar and Gourley $(1996,1997)$ to develop and calibrate a concentrated-plasticity-based threedimensional cyclic nonlinear macro-finite element model for square concrete-filled tube columns. This model was able to simulate strength and stiffness degradation due to cyclic loading. In bridge structures, plastic hinging at the column base generally forms the main energy dissipation mechanism, and findings from research in that field can be useful for high-loading applications. Large steel box or circular tubular columns are often used in bridges, and the required rotational ductility is obtained by using hollow or concrete-filled rectangular or circular tubing columns (e.g., Kawashima et al., 1992; Usami et al., 2000; Zheng et al., 2000; Susantha et al., 2002; Marson and Bruneau, 2004; Ishizawa and Iura, 2005; Mamaghani, 2008). 


\section{A.3.3 Panel Zone Behavior}

In seismic design of steel moment frames adequate shear strength and stiffness of joint panel zones is important. Krawinkler and Mohasseb (1987) showed that if panel zones are designed to be strong in shear, frame behavior is as predicted from conventional rigid frame analysis with inelastic deformations concentrated in plastic hinge regions in beams and columns. If panel zones are weak in shear, the joints become weak links in which inelastic deformations are concentrated, and the beams and columns will not develop their full bending strength under lateral loading. Based on experimental studies in the early 1970s that focused on the cyclic behavior of panel zones (Naka et al., 1969; Fielding and Huang, 1971; Krawinkler et al., 1971; Slutter, 1981; Popov et al., 1985; and Becker, 1975) it was concluded that panel zones respond in a ductile mode of deformation when subjected to unbalanced beam moments that cause high shear forces across the panel zone.

The hysteretic response of a typical weak panel zone is shown in Figure A-3. This figure shows that an elastic range, a range of gradual stiffness decrease, and a range of almost constant strain-hardening characterize the shear force-shear distortion behavior of a typical panel zone. Steel beam-to-column joints with thin panel zones may buckle in shear. This results in strength deterioration in the panel zone and stable hysteretic response.

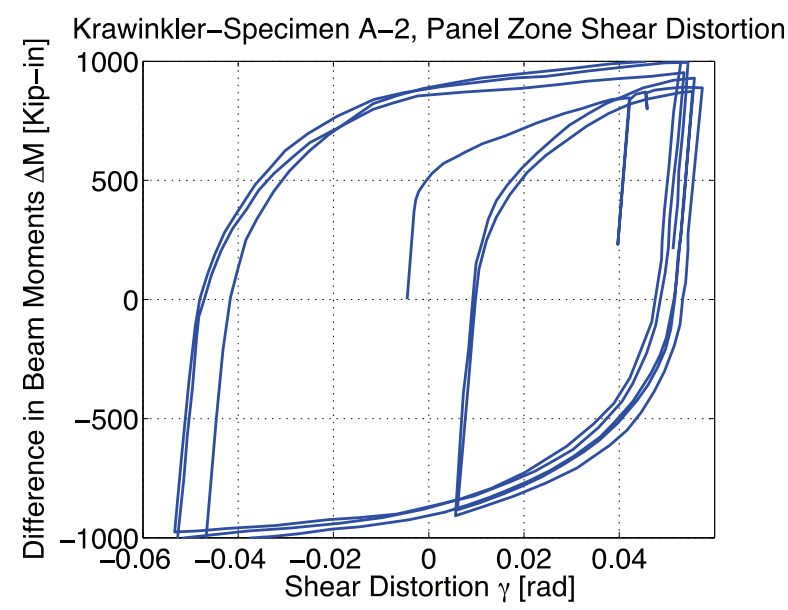

Figure A-3 Typical joint panel zone behavior (data from Krawinkler et al., 1971).

Resistance to shear forces in beam-to-column connections is provided by the panel zone, but also by the column flanges. If the column flanges are thin, severe kinking can occur at the four corners of joints when they are subjected to large shear distortions. Subsequently, high curvatures are developed at the welded beam flange connections, which can lead to crack propagation and fracture at the beam flange welds. The behavior has been confirmed in a number of analytical studies using detailed finite element modeling (El-Tawil et al., 1999; El-Tawil, 2000; Ricles et al., 2004a). For this reason, Krawinkler et al. (1971) recommended restricting shear distortion in panel zones to about four times the shear distortion at yield. Based on the 
same experimental study, "controlled" inelastic behavior of panel zones combined with beam flexural yielding, in general, improves the overall seismic behavior of steel frames. This has been confirmed by a number of analytical studies on steel moment resisting frames (Krawinkler and Mohasseb, 1987; and Gupta and Krawinkler, 1999).

Provided that column flanges remain elastic during panel zone yielding, column axial loads do not have much effect on the ultimate shear strength of the panel zone, since column flanges are able to provide axial load resistance. This is based on past experimental studies conducted by Krawinkler et al. (1971) and Popov et al. (1975). It should be pointed out that in these experimental studies the column sizes were relatively small $(\mathrm{W} 8 \times 24, \mathrm{~W} 8 \times 67)$.

Based on integrated experimental and analytical research on full scale composite RBS beam-to-column connections with deep columns (Ricles et al., 2004a), the effect of panel zone strength on the cyclic behavior of RBS connections to deep columns was evaluated. Based on this study, weaker panel zones were shown to have an increased potential for ductile fracture in the connection. Stronger panel zones resulted in an increase in the column twist and degradation in connection capacity under cyclic loading caused by beam local buckling in the RBS region. In one of the test specimens that involved a W24 $\times 131$ column and a W30 $\times 108$ beam, the ratio of the shear strength in the RBS region versus panel zone design shear strength, $V_{R B S, p} / V_{P}$, was close to 1.0, and most of the plastic deformation was concentrated in the panel zone. Fracture occurred in the heat-affected zone (HAZ) of beam bottom flange during the 5\% story drift cycle. However, a balanced panel zone design resulted in very little column twisting, no visible beam flange buckling, and almost no twisting of the RBS region compared to a design with strong panel zones (see Figure A-4).

Based on this study, balanced panel zone strength is recommended.

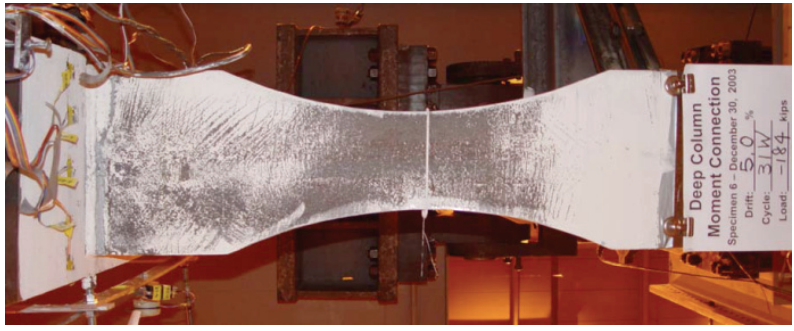

(a)

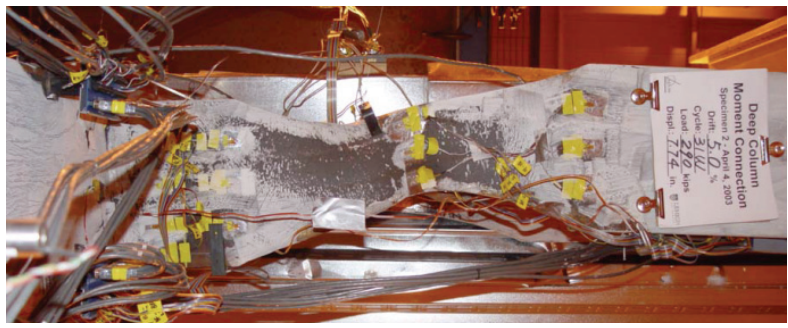

(b)

Figure A-4 Results from connection tests of RBS beams with deep columns: (a) balanced panel zone design; (b) strong panel zone design (Ricles et al., 2004a).

Similarly, Lee et al. (2005) investigated the effect of panel zone strength and beam web connection methods on seismic performance of eight RBS beam-to-column connections. Based on this study, specimens with a welded web connection and medium or strong panel zones were able to provide satisfactory plastic rotation capacity of the connection. Two specimens that were designed with a strong panel zone $\left(V_{R B S, p} / V_{p}=0.56\right)$ dissipated considerably less energy than the other specimens 
since all energy dissipation was concentrated in the RBS region, causing web local buckling and lateral-torsional buckling in the beams. This resulted in column twisting. Similar observations have been reported by other investigators (Engelhardt et al., 2000; Uang et al., 2000; Fry et al., 1997) based on experimental data for beams with RBS connections. In the experimental program by Engelhardt et al. (2000) and Uang et al. (2000) a W14×398 column (stocky column) was used with a W36×150 beam.

Choi et al. (2000) reported similar problems related to weak versus very strong panel zones based on a number of cyclic tests on free flange moment connections. FEMA 355D includes extensive information related to the effect of panel zone yielding on cyclic performance of beam-to-column connections. In general, steel specimens with a ratio $V_{p z} / V_{y}<0.6$ had virtually no panel zone yielding, and specimens with a ratio $V_{p z} / V_{y}>0.90$ experienced beam or column flange fracture rather than strength and stiffness deterioration. Beam-to-column connections with a ratio $V_{p z} / V_{y}$ between 0.60 and 0.90 achieved large inelastic rotations without any early fractures.

A number of numerical models have been proposed to simulate the hysteretic response of joint panel zones. Krawinkler et al. (1971) proposed trilinear relationships between shear force-shear distortion of the panel zone for monotonic loading based on experimental data on relatively thin column flanges. These relationships have been adopted by several design provisions, including FEMA 350, Recommended Seismic Design Criteria for New Steel Moment Frame Buildings (FEMA, 2000a), and ANSI/AISC 341-10, as a basis for computing the shear strength of panel zones. Wang (1988) has proposed similar relationships.

The model proposed by Krawinkler has been utilized in numerous analytical studies that evaluated the seismic response of steel moment resisting frames through collapse (Gupta and Krawinkler, 1999; Arbona et al., 2008; Chang and Lin, 2007; Lignos and Krawinkler, 2009; NIST, 2010; and Lignos et al., 2011a, 2011c, 2011e). In the study by Gupta and Krawinkler (1999) that summarizes the seismic response of a range of moment resisting frames that were designed for the SAC project and were modeled with and without panel zones, it was concluded that the presence of panel zones in analytical models of a moment resisting frames can result in erroneous prediction of the demands at the component level, and at the system level for certain structures.

Krawinkler et al. (1971) pointed out that a different model might be needed for joints with thick column flanges. This was confirmed by Wang (1988), who demonstrated that shear force-shear distortion relationships in Krawinkler (1971) could overestimate panel zone shear strength for panel zones with thick column flanges. Fielding and Huang (1971) proposed a bilinear model to simulate the hysteretic response of a joint panel zone. However, the Wang (1988) model generally underestimates panel zone strength regardless of column flange thickness because the effective shear area of the panel zone is calculated as $\left(d_{c}-2 t_{c f}\right) t_{c w}$ instead of $\left(d_{c}-t_{c f}\right) t_{c w}$. Charney and Downs (2004) 
compared the Krawinkler (1971) model with the "Scissors" model for predicting panel zone shear force-shear distortion with detailed finite element analysis of full beamcolumn subassemblies modeled in ABAQUS. These subassemblies were part of tests conducted on the SAC project (FEMA 350; Ricles, 2002) indicating that simulated response of joint panel zones is predicted fairly well with both mechanical models. However, the Scissors model is limited to use in steel frames with equal bay widths and equal story heights.

Kim and Engelhardt (2002) proposed a numerical model with a hysteretic response based on Dafalias (1975) bounding surface theory and utilizing the Cofie and Krawinkler (1985) rules for movement of the bound line. This model was shown to simulate the hysteretic response of bare joint panel zones reasonably well based on comparisons with available experimental data (Krawinkler et al., 1971; Popov et al., 1985; Becker, 1971; Slutter, 1981). The same model was also validated with available experimental data of composite steel beam-to-column subassemblies (Lee, 1987; Engelhardt et al., 2000). However, this model is not able to simulate strength deterioration in the panel zone due to shear bucking or fracture of the column or beam flanges at the corner of the panel zone.

More recently, Castro et al. (2005) developed a new panel zone model that includes both shear and bending deformations. This model considers an accurate assessment of the shear stress distribution through the panel depth that allows a realistic representation of the spread of plasticity. This model was validated with monotonic tests of bare steel and composite frame structures that were modeled numerically using finite elements. Krishnan and Hall (2006) developed a three-dimensional element for simulating the response of a panel zone as part of steel frame buildings in three dimensions. The model consists of two orthogonal panels forming a cruciform section, and each panel is allowed to yield and strain-harden in shear. However, no comparison with experimental data is available for this model in the literature.

\section{A.3.4 Strong-Column-Weak-Beam Design}

Steel moment frames are designed using a strong-column-weak-beam (SCWB) design concept. This concept encourages plastic deformation in the beams and discourages plastic deformation in the columns. Based on integrated experimental and analytical research, Schneider et al. (1993) demonstrated that weak-column-strong-beam (WCSB) frames concentrate inelastic deformations in individual stories, and as a consequence, localized seismic demands are much larger in such structures.

ANSI/AISC 341-10 seismic provisions control SCWB behavior by balancing the plastic capacity of the beams with the plastic capacity of the columns:

$$
\sum M_{p c}^{*} \geq \sum M_{p b, e}
$$


where $M_{p c}^{*}$ is the nominal flexural strength of the columns reduced for the presence of axial load, and $M_{p b, e}$ is the expected plastic moment capacity of the beams determined using expected beam yield strength accounting for strain-hardening effects consistent with anticipated plastic deformations. It is recognized that this equation does not prevent column yielding, which can still occur because of: (1) strain hardening during inelastic deformation; (2) material variability; (3) composite effects; (4) variation in geometric corrections needed to transfer beam moment capacities to the column; and (5) varying locations of inflection points in beams and columns during seismic loading.

Krawinkler (2000) reported that column plastic hinging may not be detrimental to global response unless column hinging leads to undesirable story mechanisms. However, column hinging will clearly affect the state of stress and strain at the beamto-column connection and, therefore, the likelihood of fracture at weldments.

Nakashima and Sawaizumi $(1999,2000)$ suggested that column strength after yielding should be greater than $120 \%$ of the beam plastic moment capacity, $M_{p}$, to account for strain-hardening and control the adverse effects of concentration of plastic deformation. Recently, a full-scale collapse test of a four-story steel frame structure designed based on current Japanese seismic provisions was conducted at the EDefense shaking table in Japan (Suita et al., 2008). This structure collapsed in a firststory sidesway mechanism because of increased beam strength due to strain hardening, slab effects, and material variability. This resulted in column strength deterioration due to local buckling.

In this test, a SCWB criterion was employed. In Japan, the ratio used in the SCWB relation is 1.5. Based on numerical studies conducted by Lignos et al. (2011d) it is possible to prevent the first story collapse mechanism of the same structure if a SCWB ratio larger than 2.0 is employed. This agrees with other analytical and experimental studies in the literature (Nakashima and Sawaizumi, 1999, 2000; Gupta and Krawinkler, 2000; Ibarra and Krawinkler, 2005; NIST, 2010; Lignos et al., 2011a; Zareian et al., 2011).

\section{A.3.5 Lateral Bracing of Beams and Columns}

ANSI/AISC 341-10 seismic design provisions require that lateral support be applied at all plastic hinge locations, and that additional supports be applied at intervals of $L_{b}$ from these locations. $L_{b}$ should be less than or equal to $2500 r_{y} / F_{y}$, in which $r_{y}$ is the radius of gyration of a beam with respect to its weak axis and $F_{y}$ is the yield strength of the steel material. Many post-Northridge connections, including RBS, coverplate, and haunch connections, move the plastic hinge away from the face of the column. Application of the AISC LRFD requirements to these connections requires lateral support at these hinge locations. As discussed in FEMA 355D, it may be difficult to provide this support because research has shown that attachments to yielding flanges can cause local fractures. Based on reconnaissance reports from the Kobe earthquake, 
little evidence of buildings that sustained serious damage due to lateral-torsional buckling exists. Furthermore, the Japanese seismic provisions permit longer unbraced lengths than those employed in the U.S. $\left(L_{b} \leq 130 r_{y}\right)$ for comparable steel material properties.

Moreover, Lignos and Krawinkler $(2009,2011)$ evaluated the effects of lateral bracing on seismic performance of more than 300 beam-to-column connections tested worldwide. Multivariate regression analysis was performed after calibrating the hysteretic response of the modified Ibarra-Krawinkler deterioration model (Ibarra et al., 2005; Lignos and Krawinkler 2009; 2011) with deduced moment rotation hysteretic diagrams of these connections. It was concluded that the web slenderness ratio, $h / t_{w}$, had the greatest effect on connection performance, regardless of the connection type and the presence of additional lateral bracing. This agrees with past studies (Uang and Fan, 1999). Additional lateral bracing was not very effective in improving the pre-capping plastic rotation of steel beams, particularly for beams other than RBS. For RBS beams, a decrease in $L_{b} / r_{y}$ has a small effect on pre-capping plastic rotation, but additional lateral bracing reduces the rate of cyclic deterioration.

This can be seen in Figure A-5, which shows the deduced moment rotation diagram of two nominally identical RBS connections with W30×99 steel beams, in which the insertion of additional lateral bracing close to the RBS region had a small, but measurable improvement in hysteretic behavior. In the figure, it can also be seen that specimens without additional lateral support might still provide adequate seismic performance. Similar observations were reported from extensive finite element analysis (Kwasniewski et al., 1999; Uang and Fan, 1999).

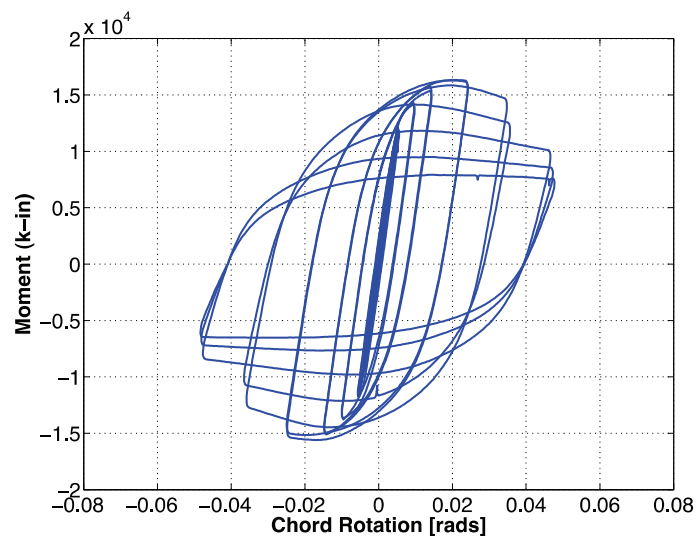

(a) Without additional bracing near the RBS

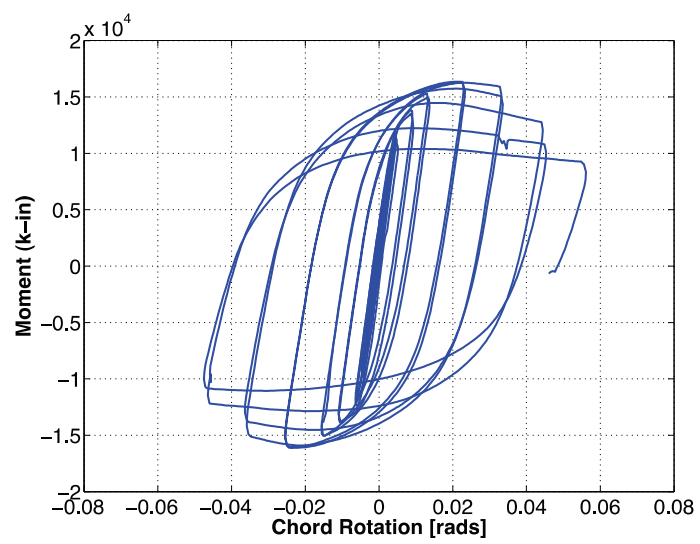

(b) With additional bracing near the RBS

Figure A-5 Effect of additional lateral bracing near reduced beam sections (data from FEMA 355D). 


\section{A.3.6 Effect of Composite Slabs on Cyclic Response of Beam-to-Column Connections}

Composite slabs are commonly used with beams supporting gravity loads, but are generally not considered in seismic design. Detailed finite element simulations on various full-scale beam-to-column subassemblies by Kwasniewski et al. (1999) showed that axial restraint provided by the slab in the subassemblies resulted in significant reduction in the post-buckling deterioration of steel beam-to-column connections. Concrete floor slabs in buildings are expected to provide this axial restraint, and this is viewed as a beneficial effect in reducing the adverse effects of lateral-torsional buckling. Furthermore, a concrete slab that is integral with the beam and the column would be expected to restrain deformation and reduce the deterioration effects of twisting and out-of-plane movement.

Assessment of the effect of composite slabs on the seismic performance of steel beamto-column connections has been largely accomplished through the use of cruciform subassemblies. Due to beam boundary conditions in these subassemblies, however, it is recognized that the axial restraint provided by the slab is underestimated. This has been shown in tests conducted on full scale structural systems by Cordova and Deierlein (2005) using a 4-story composite frame subjected to quasi-static loading. Similar conclusions have been drawn from experimental research conducted as part of a joint US-Japan research program (Leon and White, 1998).

The effect of composite floor slabs on the seismic performance of beam-to-column connections depends on the configuration of the subassembly. There are differences in behavior between perimeter connections with a composite slab, interior connections with a composite slab, and connections that involve a gap between the composite slab and the column face. For a composite beam in a perimeter steel moment resisting frame, the effect of the composite slab on the rate of cyclic deterioration in the two loading directions is not significant (Ricles et al., 2004a). This is illustrated in Figure A-6.

For composite steel beams that are part of an interior moment resisting frame, the effect of the composite slab on the rate of cyclic deterioration becomes more important. This is illustrated in Figure A-7, which shows the results of two cyclic tests on a W36 $\times 150$ beam with RBS connections (Engelhardt et al., 2000). It should be noted that these tests had an additional lateral support near the beam loading points.

For composite steel beams with a gap between the slab and the column face, Tremblay et al. (1997) and Lignos et al. (2011e) showed that the bending strength of the beam in the two loading directions is nearly identical. 


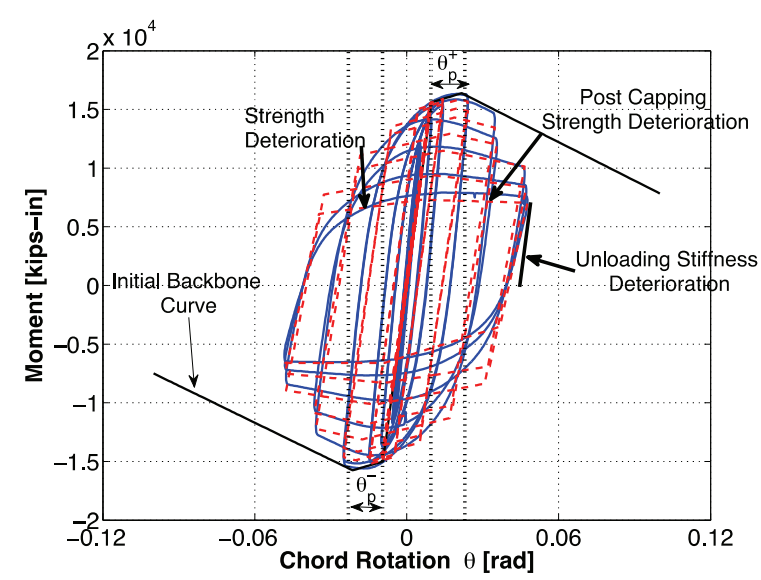

(a) Bare steel beam

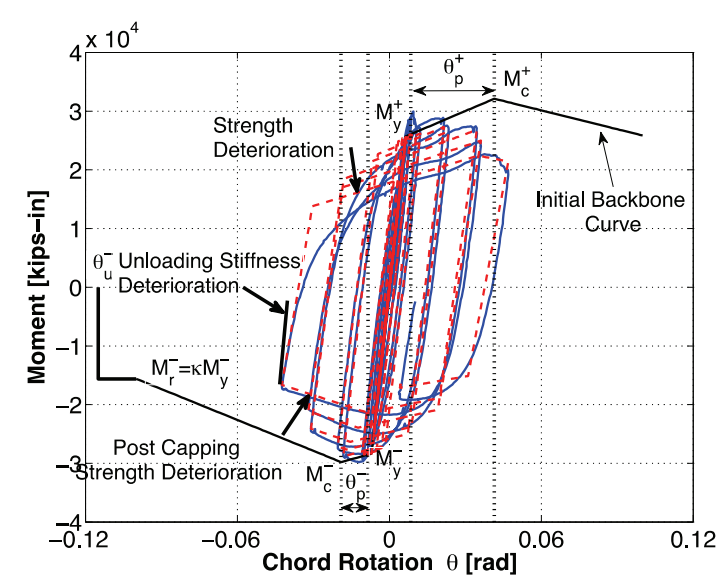

(b) Composite beam

Figure A-6 Comparison of the hysteretic response of bare steel and composite beams in a perimeter frame (data from FEMA 355D and Ricles et al., 2004a; calibrations from Lignos et al., 2011e).

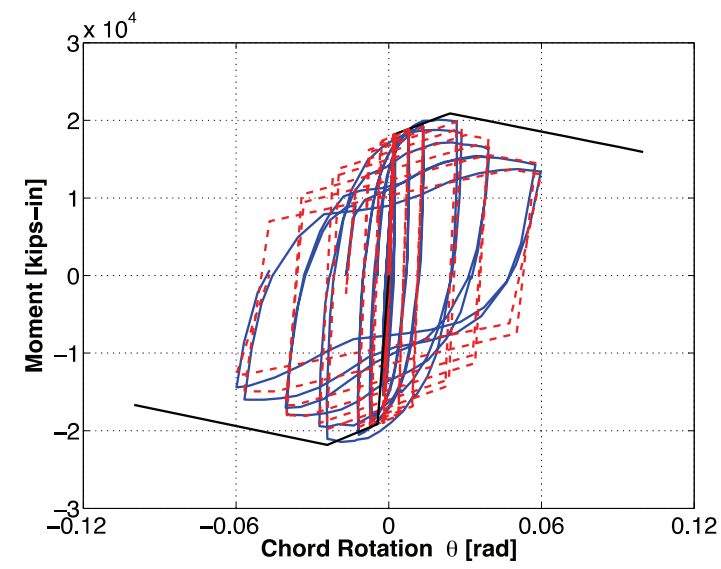

(a) Bare steel beam

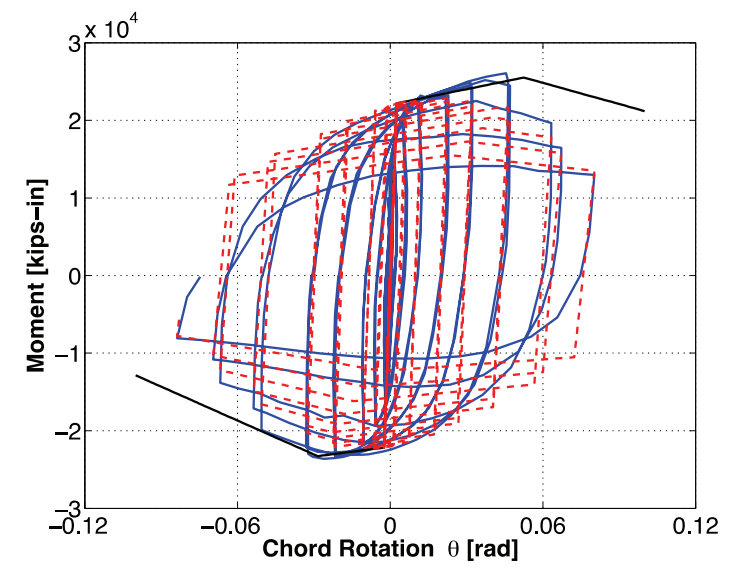

(b) Composite beam

Figure A-7 Comparison of the hysteretic response of bare steel and composite beams in an interior frame (data from FEMA 355D; calibrations from Lignos et al., 2011e).

These observations agree with prior experimental work conducted by a number of investigators (Gross et al., 1999; Leon et al., 1998; Engelhardt et al., 2000; Ricles et al., 2000; Venti et al., 2000). For deeper beams, the composite slab does not make a significant contribution to beam resistance because the slab is relatively thin and the beams are relatively deep, and the number of shear connectors usually only sufficient for partial composite action (FEMA, 2000e; Ricles et al., 2004a). Due to the shift of the neutral axis of the beam toward the slab, the presence of a composite slab reduces the strain demand, and the possibility of fracture, at the top flange.

There are cases in which composite action has a negative effect. Ricles et al. (2000) demonstrated this experimentally with tests on welded-flange-welded-web postNorthridge connections with shear connectors distributed along the length of the beam length. In some specimens, the composite beam-to-column connection fractured through the beam in the region of plastic deformation of the beam flange. The 
fracture started at a shear connector weld, and occurred well away from the flange weld and weld access hole. A summary of the effects of composite slabs on beam-tocolumn connection performance, including deterioration parameters and modeling recommendations for composite beams, is discussed in Lignos et al. (2011e).

\section{A.3.7 Effect of Loading History on Seismic Performance of Beam-to- Column Connections}

Load and deformation history can have a significant impact on the performance of moment resisting connections (FEMA 355D; Clark et al., 1997; Lignos and Krawinkler, 2009; Krawinkler, 2010; Lignos and Krawinkler, 2011a). As part of FEMA 355D, Yu et al. (2000) tested four full-scale, welded web, RBS connections to study the effect of loading history on performance. Two different loading protocols were used in the SAC program. The symmetric loading protocol (Clark et al., 1997) used for the majority of tests conducted under the SAC-Phase II program, and the SAC near-fault protocol, which contains a pulse reversal in the loading history. Sample results from these tests are shown in Figure A-8. The study concluded that all four connections behaved well, and the specimens tested with the near-fault protocol were able to reach $70 \%$ more plastic deformation capacity than the specimens tested with the symmetric loading protocol.

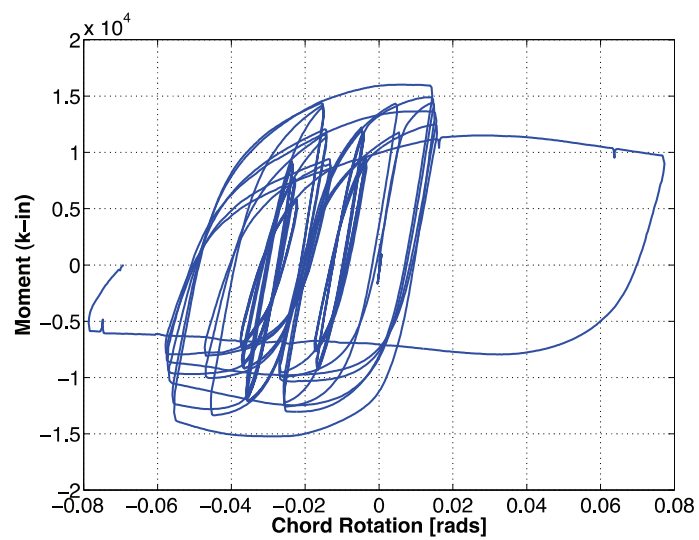

(a) Near-fault loading protocol

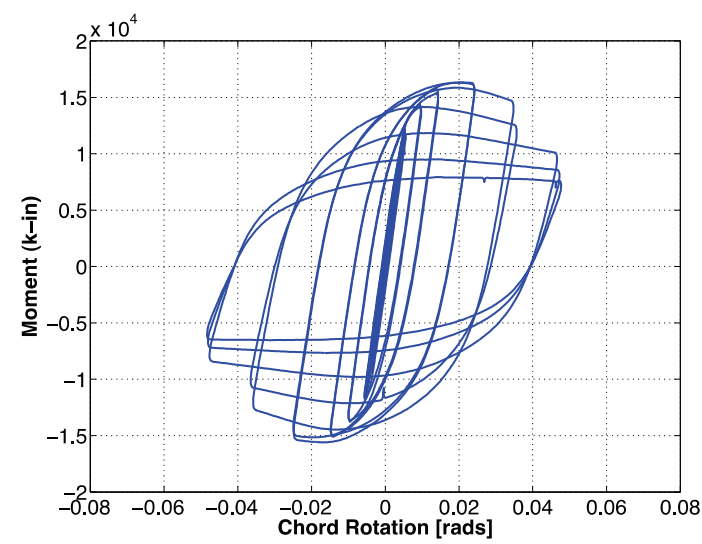

(b) Symmetric loading protocol

Figure A-8 Effect of loading history on hysteretic response of nominally identical RBS beam-to-column connections (data from FEMA 355D).

Deterioration resulting from local buckling appears to be most significant in reversed and repeated inelastic cycles after initial buckling has been noted (Castiglioni et al., 1998). Recent experimental and analytical studies related to collapse assessment of moment resisting frames (e.g., Lignos and Krawinkler, 2009; Lignos et al., 2011a) have demonstrated that component response depends on the performance level of interest. Components responding at or near collapse will have different response histories than components responding to design level events. In many cases the importance of cyclic deterioration diminishes because of "ratcheting" of the structural 
response, meaning that lateral deformations (story drifts) increase in one direction and load reversals become less significant.

\section{A.4 Research on Column-to-Foundation Connections}

Column base plates can be categorized as exposed or embedded. Exposed column base plates are considered representative of a pinned support condition and embedded column base plates are considered representative of a fixed support condition. Typical examples for each case are shown in Figure A-9. Detailed reviews on this topic are available in the literature (Dewolf and Ricker, 1990; Hensman and Nethercot, 2000; Fahmy et al., 1999, 2000; Grauvilardell et al., 2005; and Gomez, 2007). This discussion focuses on column bases attached to moment resisting frames.

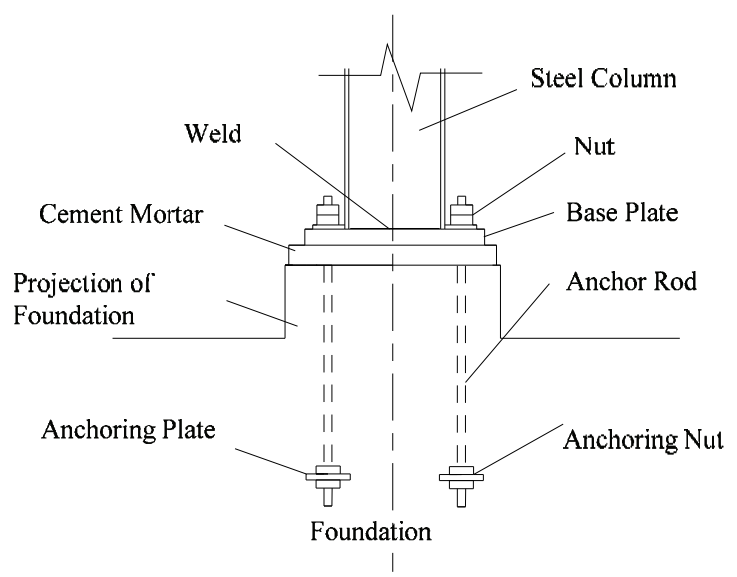

(a) Exposed base plate

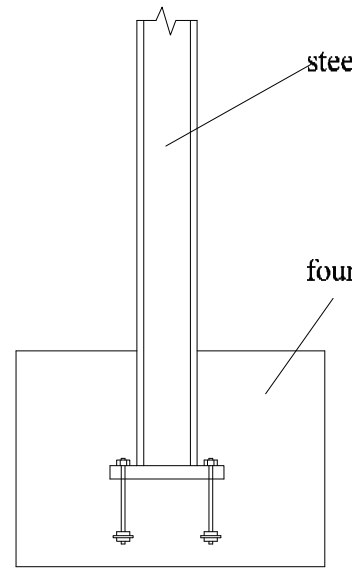

(b) Deeply Embedded
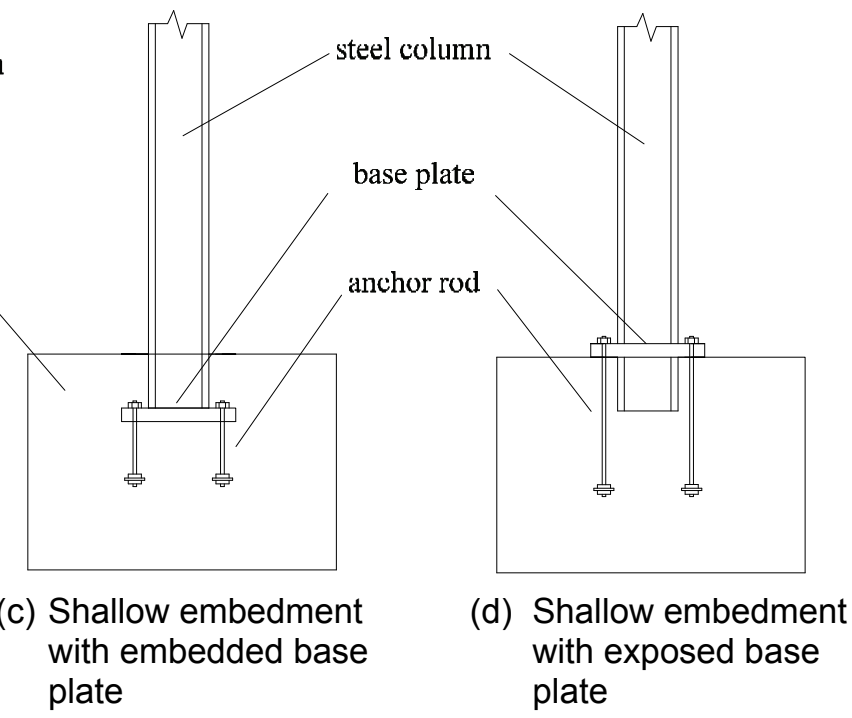

Figure A-9 Exposed and embedded column base plates (Grauvilardell et al., 2005). 


\section{A.4.1 Exposed Base Plates}

In most exposed base plate connections, the goal is to attain a pinned condition; thus a thin steel base plate is welded to the end of the column for the transfer of axial compression/tension and shear forces to the foundation. Analytical and experimental studies (Galambos, 1960; Astaneh et al., 1992; Burda and Itani, 1999; Hitaka et al., 2003; Fahmy, 1999; Lee and Goel, 2001; Gomez, 2007) have demonstrated that most column bases exhibit some level of fixity. When exposed column bases are assumed to respond as fixed supports, however, they resist the applied loads only after large deformations that were neither modeled nor considered in the design. This resulted in larger than expected story drift ratios in steel frame structures, and sometimes structural collapses, as summarized in reconnaissance reports from recent earthquakes (Northridge 1994; Kobe 1995). Exposed column base plates in Grauvilardell et al. (2005) are categorized according to: (1) base plate behavior; (2) amount of restraint provided; (3) steel failure mode; (4) concrete failure mode; and (5) energy dissipation capacity.

Early research on exposed base plates focused on axially loaded conditions and issues pertaining to base plate bearing on the concrete footing (Hawkins, 1968a, 1968b; Dewolf, 1978; Murray, 1983). Subsequent studies examined the seismic response of base connections and led to the development of Steel Design Guide 1: Column Base Plates (AISC, 1990) and the design procedures developed by Drake and Elkin (1999) that are commonly used for determining base plate size, thickness, and anchor rod dimensions and configuration. These guides were based on experimental studies conducted by Dewolf and Sarisley (1980) and Thambiratnam and Paramasivam (1986) that investigated base plate yielding, concrete crushing and anchor rod fracture as controlling failure mechanisms in exposed column base plate connections.

Investigators in the U.S. agree that exposed base plates designed using ANSI/AISC 341-10 seismic provisions will have intermediate thickness and semi-rigid stiffness and strength. Lee and Goel (2001) conducted four tests on column connections and expressed the possibility that base plates designed using current methods may behave more rigidly than expected, resulting in fracture of the anchor rods in tension while not attaining the yielding of the base plate that is intended. The same behavior was shown in an earlier experimental study by Astaneh et al. (1992).

A thin column base plate can be characterized by ductile response since most of the inelastic behavior is concentrated in the base plate. A thick column base plate can be characterized by non-ductile response due to fracture of anchor rods unless the rods have been designed to provide ductile behavior (Sato, 1987; Balut and Moldovan, 1997). In order to achieve this, the ratio of the yield strength divided by the tensile strength of the bolt material (noted as yield ratio) must be smaller than the ratio of the threaded and non-threaded cross-sectional areas of the bolt. This has been confirmed by prior experimental studies on exposed base plates (Sato, 1987; Hitaka et al., 2003; 
Cui et al., 2009; Gomez, 2007). Additional deformation capacity at the column base is beneficial for a steel moment resisting frames based on recent analytical studies by Lignos et al. (2011d) that investigated the effect of column base behavior on the collapse capacity of steel moment resisting frames subjected to extreme earthquake loading.

In the case of weak-column-strong-connection details, a plastic hinge forms in the column. Depending on the weld metal toughness, however, it is possible for premature fracture of the column base welds to occur. This was confirmed in experimental studies conducted by Fahmy et al. (2000) and Myers et al. (2009). In these studies, specimens with toughness-rated weld metal sustained about twice the drift ratios prior to fracture compared to specimens with non-toughness rated weld metal (5\% versus $2.5 \%$, respectively). Furthermore, test specimens with complete joint penetration (CJP) weld details that were tested by Burda and Itani (1999) showed greater deformation capacity prior to fracture than those with fillet weld details. Complete joint penetration welds failed at drift ratios between $7 \%$ and $13 \%$, while fillet welds failed at drift ratios between $2 \%$ and $9 \%$.

In the case of strong-column-weak-connection details the potential for non-ductile behavior is recognized through a number of brittle failure mechanisms including crushing of the concrete and anchor rod fracture (DeWolf and Sarisley, 1980; Picard and Beaulieu, 1985; Thambiratnam and Paramisivam, 1986; Astaneh et al., 1992; Jaspart and Vandegans, 1998; Burda and Itani, 1999). Along with cracking of base plate welds, these failure mechanisms are classified as non-dissipative energy mechanisms (Grauvilardell et al., 2005).

Depending on the level of axial load with respect to the ultimate bearing stress in the concrete foundation, three major failure modes have been observed (Wald et al., 1995; Stamatopoulos and Ermopoulos, 1997; Balut and Moldovan, 1997). When low axial loads are applied, the predominant failure occurs when anchor rods yield or when a plastic mechanism forms in the base plate. In the case of medium axial loads, the anchor rods yield and the concrete reaches its bearing capacity. Under high axial loads, the primary failure mode occurs when the concrete exceeds its ultimate bearing capacity.

Gomez (2007) studied the behavior of exposed column base plate connections using realistic prototypes developed based on design approaches that were current at the time. Results indicated that the characterization of anchor rod forces for design is relatively accurate, but an assumption that the capacity of the entire connection is governed by a "first-yield" failure of any one connection component is significantly conservative with respect to the ultimate strength observed in the tests. Also, longer shear lugs yielded higher shear capacities on a per unit basis, as shorter shear lugs caused higher local bearing stresses and increased the likelihood of early crack initiation. 
Based on these observations, Gomez (2007) recommended a coefficient of friction value of 0.45 for use in design, which is comparable to the design value of 0.40 given in ACI 349, Code Requirements for Nuclear Safety Related Concrete Structures and Commentary (ACI, 2006), and proposed a new design procedure based on a plastic mechanism approach for predicting the ultimate strength capacity of the overall connection.

\section{A.4.2 Embedded Column Base Plates}

In embedded base plate connections, the goal is to attain a rotationally fixed condition. Embedded base plate configurations include: (1) deeply embedded column and base plate; (2) shallow embedment with an embedded base plate; and (3) shallow embedment with an exposed base plate. Anchor rods in all configurations are designed for construction loads and they have a significant influence in the overall behavior only in the shallowly embedded column bases. Most of column base research in the U.S. has focused on exposed column-base plate connections, but shallow or deeply embedded column-base plate connections have been common in both steel moment resisting frames and braced frames in high regions of high seismicity. Embedded column base plates in Grauvilardell et al. (2005) are categorized in accordance with: (1) length of embedment; (2) column position in the frame (i.e., interior versus end column); (3) failure mechanism; and (4) deformation capacity.

Based on integrated experimental and analytical studies conducted by Pertold et al. (2000a, 2000b) wide-flange columns are considered fixed when the embedment lengths range from $1 D$ to $2 D$, with $D$ being the lateral dimension of the column cross section in the plane of bending. Nakashima and Igarashi (1986) and Morino et al. (2003) indicated that for steel tube box columns, the embedment length should be at least $2 D$. In combination with a column base flexural strength greater than 1.3 times the plastic moment capacity of the column, this guarantees plastification of the steel section and full rigidity of the column base (Hitaka et al., 2003; Cui et al. 2009). Full hysteretic loops are often observed for deeply embedded column base plates. The axial strength of the base plate connection is governed by two mechanisms: (1) for compression, the bearing of the end of the column on the bottom of the concrete foundation and (2) for compression or tension, the bond strength between the steel and concrete.

The concept of shallowly embedded base plates including two main design procedures was developed in Japan (Nakashima, 1996; Morino et al., 2003) for box columns and concrete-filled steel tube (CFT) columns. By embedding the column at a depth of one times its width, substantially smaller base plates are required and fewer and smaller anchor rods are required in comparison with exposed base connections. Full plastification of the steel column base has been achieved when thicker embedded base plates together with a larger number of anchor rods are used (Nakashima, 1996). 
Cracking of the concrete is the controlling failure mechanism when weaker base plates or no anchor rods are used, however, horizontal reinforcement does help to reduce cracks on the top of the concrete (Nakashima, 1996). Yielding of the anchor rods and cracking of the welds at the base plate typically follow cracking of the concrete around shallowly embedded column bases. These failure mechanisms can occur simultaneously with failure of the concrete depending on the relative strength between the concrete and the strength of each mechanism (Nakashima, 1996). No guidelines exist related to this relationship.

End columns in moment resisting frames can be subjected to large tension forces due to global overturning moments. Also, if the edge distance between the column and external face of the concrete foundation is not sufficient, sudden deterioration of the connection can occur when bearing forces due to column bending toward the free edge of the foundation causes the concrete to fail. An experimental study by Nakashima and Igarashi (1986) indicated that the minimum edge distance should be at least 1.5 times the depth of the column cross section in the plane of bending. For the case of embedded base plate connections on interior columns, past research has generally investigated only the general parameters that affect behavior, such as embedment length and reinforcement methods. The hysteretic behavior of such connections is generally symmetric.

\section{A.4.3 Research on Column Splices}

Steel column splice connections that are part of moment resisting frames should be designed to develop the full bending and shear strength of the column sections. Column splices can be categorized based on the connection type (welded versus bolted column flange). Column flange splices should be designed to preclude net section fracture, block shear failure, and bolt pull-through failure of the column flange or splice plates.

In preliminary design, the point of inflection in steel beams and columns of moment resisting frames is typically assumed to be at mid-length. In steel columns, inflection points can shift substantially from locations indicated in an elastic analysis due to inelastic behavior and moment redistribution within the frame. Designers sometimes locate the column splice near an inflection point based on elastic analyses and design the splice for relatively small bending moments. Popov and Stephen (1977a, 1977b) tested a set of W14×176 and W14×320 columns with $1 / 16$ inch and 1/4 inch gaps in the splices to recognize that cut surfaces do not make perfect full contact. Partial penetration welds were used in the splices. Results indicated that a lack of perfect contact at compression splices is not serious, provided the gaps are carefully shimmed. In tension, all failures occurred through the welds, indicating a brittle failure mode. Lack of uniformity of the weld size suggested the need for a visual inspection of such welds if the members are designed to transmit tensile forces. Bruneau et al. (1987) and Bruneau and Mahin (1991) concluded the same after testing 
large-scale spliced columns (W14×665 to W14×500) in flexure. Based on these tests, the moment curvature relationship remained nearly linear up to about $60 \%$ of the nominal plastic moment of the smaller column section at the splice, and then the weld fractured in a brittle manner. Full penetration welds exhibited satisfactory strength and ductility.

Due to large bending and axial stresses at column splices, FEMA 350 recommends that weld filler metals with rated notch toughness be used, and that runoff tabs be removed. Where complete joint penetration (CJP) welds are used, removal of the backing is not judged to be necessary. Welded splices of columns conforming to ASTM A913, Grade 65 steel should be made with filler metals capable of depositing weld metal with a minimum tensile strength of $80 \mathrm{ksi}$. Inelastic analyses have shown that columns that are not part of the seismic-force-resisting system participate in distributing the seismic forces between floors. Even gravity columns with pinned beam connections can develop large bending moments and shears due to non-uniform drifts in adjacent floor levels. For this reason, it is important that splices of gravity columns be adequate to develop shear forces corresponding to the development of plastic hinges at each end of the columns in both orthogonal directions.

Snijder and Hoenderkamp $(2006,2008)$ developed strength and stiffness criteria for end plate column splices with respect to column stability. They concluded that column splices should be designed with sufficient strength to resist normal forces and second order bending moments and accompanying shear forces. A stiffness requirement was derived on the basis of allowing a maximum reduction in the Euler buckling load of $5 \%$ due to the presence of a splice. A limited number of experimental tests on HE100A columns showed that butt-plate splices can have a negative influence on the load carrying capacity of slender columns (Snijder and Hoenderkamp, 2008). Based on these tests, Coelho and Bijlaard (2008) and Coelho et al. (2010) analyzed the behavior of spliced columns from a stability standpoint and implemented a set of parametric studies by varying: (1) splice location and rotational stiffness; (2) change in the column section serial size; and (3) column end-restraint stiffness coefficients. Simple relationships were developed for use as a basic stiffness design criterion for column splices.

\section{A.5 Structural System Investigations}

This section presents major findings related to investigations on seismic response of steel moment resisting frame systems. Emphasis is placed on available simulation models for modeling of inelastic response of steel beams and columns. Other issues include the range of axial load ratios in steel moment frames and the assessment of collapse potential. 


\section{A.5.1 Steel Building Simulation Models}

Inelastic modeling of moment frame systems primarily involves component models for flexural members (beams and columns) and their connections. For systems that employ "special" moment frame capacity design principles, as defined in ASCE/SEI 7-10, Minimum Designs Loads for Buildings and Other Structures (ASCE, 2010), inelastic deformations should occur at flexural hinges located in the beams and column bases. The present discussion is limited to consideration of rigidly connected steel frame structures in which the primary effect is yielding of members due to combined axial load and bending moments. In frames that do not meet special moment frame requirements of ASCE/SEI 7, inelastic effects may occur in other locations, including member shear yielding, connection failure, and member instabilities due to local or lateral-torsional buckling. Technical Brief No. 4, Nonlinear Structural Analysis for Seismic Design: A Guide for Practicing Engineers (NIST, 2011) summarizes approaches for modeling the behavior of steel moment frames under such conditions. Modeling recommendations for steel structural components are also summarized in the PEER/ATC 72-1 Report, Modeling and Acceptance Criteria for Seismic Design and Analysis of Tall Buildings (PEER/ATC, 2010) and in ASCE/SEI 41-06 Seismic Rehabilitation of Existing Buildings (ASCE, 2007).

Inelastic structural component models can be classified by the way that inelastic behavior is distributed through the member cross-section and along its length. Figure A-10 shows a comparison of five idealized model types for simulating the inelastic response of beams-columns as summarized in NIST (2011). The concentrated plasticity models shown in the figure are essentially zero length elements that simulate the moment-rotation relationship of a steel component. The distributed plasticity models shown in the figure include finite length hinge models with designated hinge zones at the member ends in which cross sections are characterized through either nonlinear moment-curvature relationships or explicit fiber-section integrations based on the assumption that plane sections remain plane.
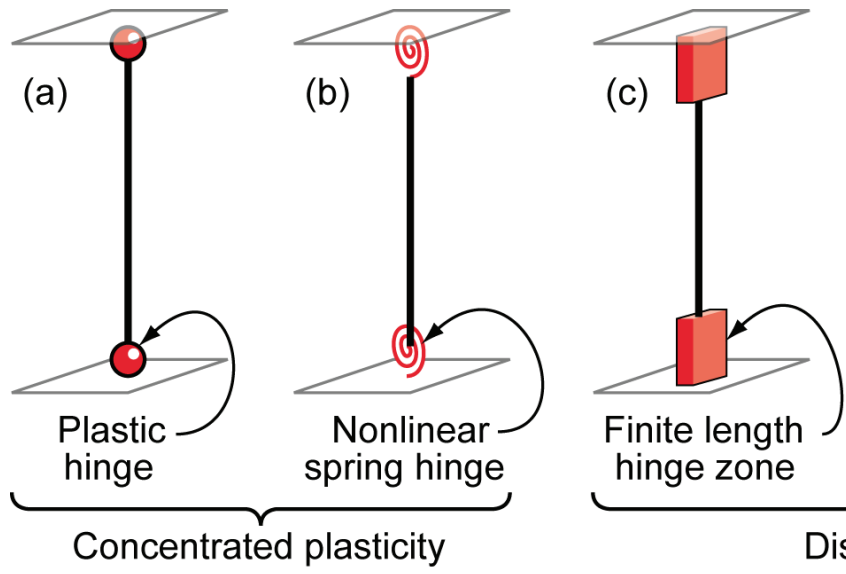

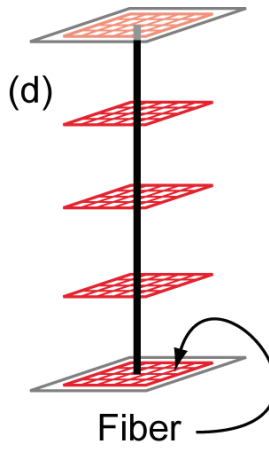

section (e)

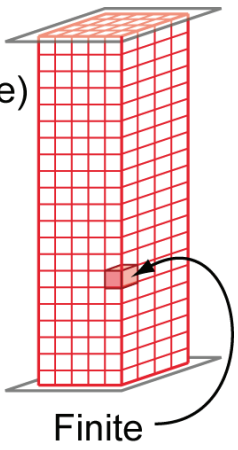

element

Distributed plasticity 
The fiber formulation (Figure A-10d) involves models that distribute plasticity by numerical integration through the member section and along the member length. These models use displacement or force interpolation functions for numerical integration of cross section parameters (Kunnath et al., 1990; Spacone et al., 1996). Typically, strain demands are benchmarked against concentrated hinge models for which rotation acceptance criteria are more widely reported. Finally, the numerical model in Figure A-10e involves discretization along the member length and through the cross section into finite elements with constitutive laws. These models offer versatility for simulating the component hysteretic response. However, model parameter calibration and computational time is a challenge. It should be pointed out that distributed plasticity models are able to capture reductions in member stiffness prior to full plastification, which can affect second-order deformations and internal forces and moments. A review of a number of analysis models discussed herein can also be found in FEMA P-440A (FEMA, 2009a) and Lignos and Krawinkler (2009).

Gioncu and Petcu (1997), Möller et al. (1997) and Anastasiadis et al. (2000) introduced the yield-line concept to model the buckled shapes observed in experiments. They constructed yield-line models and used this plastic mechanism to determine rotation capacity of European H-section beams. Lee and Stojadinovic (2004) developed a new cyclic yield-line plastic hinge model for estimating connection rotation capacity based on a similar concept. The model considers postpeak connection strength degradation due to local and lateral-torsional buckling. This limit state is used to establish a connection rotation limit similar to the limit used in FEMA 350. In order to predict crack initiation at the creases of local buckles in the plastic hinge, the model also considers low-cycle fatigue crack initiation based on a cumulative local strain concept at the critical yield line. A more detailed discussion about these models can be found in Mazzolani and Gioncu (2002) and Lignos and Krawinkler (2009).

\section{A.5.1.1 Phenomenological Models}

A number of analytical models have been developed to simulate the inelastic action of steel components subjected to cyclic bending moments. The focus of this section is on models that are able to simulate component deterioration for steel frame structures. Foliente (1995) summarized the use of the Bouc-Wen model (Bouc 1967; Wen 1980) and its derivatives to account for component deterioration based on modifications that were implemented by Baber and Noori $(1985,1986)$ and Reinhorn et al. (1995). About 13 parameters need to be specified for the Bouc-Wen model and its modifications in order to be able to simulate the hysteretic response of structural components. It should be pointed out that few of these parameters can be related to engineering quantities under control by the designer. Values for these parameters have been obtained for specific cases through system identification. The great advantage of this general model is its versatility, since almost any hysteresis behavior 
can be modeled in some manner. But its complexity is a great disadvantage along with its lack of relation to engineering design parameters.

Sivaselvan and Reinhorn (2000) developed a versatile smooth hysteretic model that includes stiffness and strength degradation and pinching, derived from inelastic material behavior based on the earlier models by Iwan (1966) and Mostaghel (1999). This model was also used for numerical simulations of steel frame structures as part of the SAC project. It is not evident in the literature how strength cap and post-capping behavior are considered by the Sivaselvan and Reinhorn model and its previous variations. At about the same time, Song and Pincheira (2000) developed an analytical model that incorporates a strength cap and post-capping behavior, but does not incorporate cyclic strength deterioration. The model deterioration rules are described in detail in Song and Pincheira (2000). A residual strength path is incorporated in the model.

In ASCE/SEI 41-06, structural components with reliable ductility are modeled with a multi-linear approximation similar to that shown in Figure A-11a. This model has a strength cap (Point $\mathrm{C}$ ) beyond which strength drops rapidly (Points $\mathrm{C}$ to $\mathrm{D}$ ) to a much reduced residual value that is maintained until the deformation reaches a specified value (Point E), where the strength drops to zero. This model was intended to be used for static pushover analysis and not for inelastic dynamic analysis. Thus the model does not include any hysteretic rules for cyclic deterioration. Additionally, even in static analyses the stiffness singularity at Point E causes many numerical stability problems.
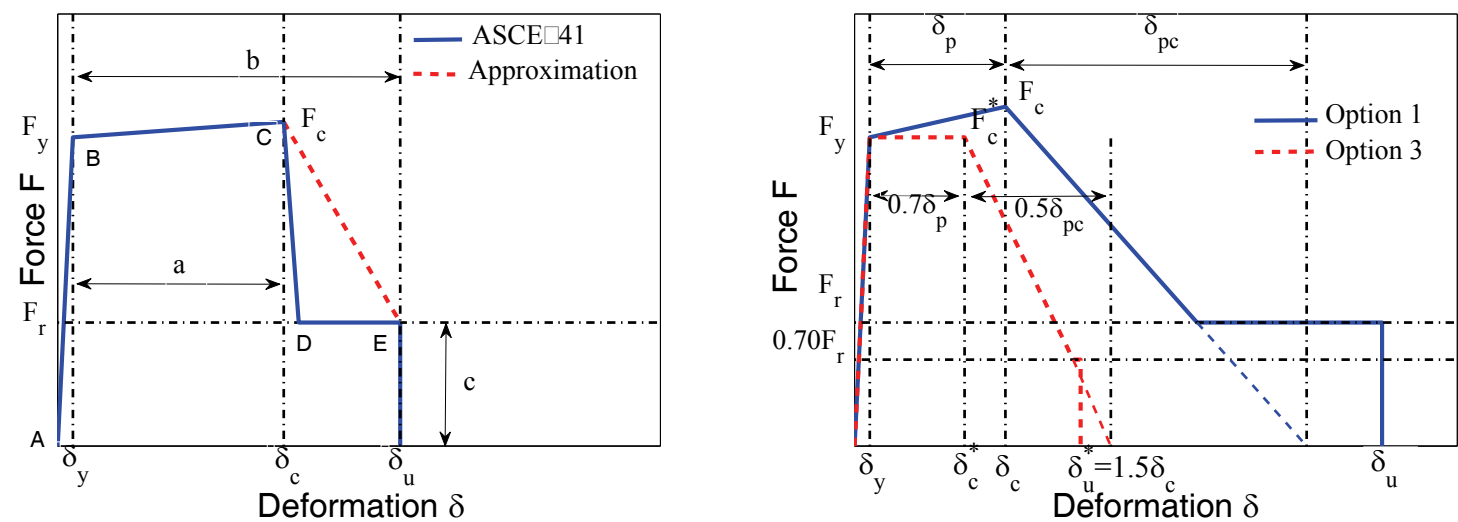

Figure A-11 Comparison between ASCE/SEI 41-06 and modeling options based on PEER/ATC-72-1 (Lignos et al., 2011e).

Figure A-11b shows a backbone curve for a steel component that does not account for in-cycle deterioration (Option 1) and the parameters that are needed to specify this curve are based on multivariate regression relationships proposed by Lignos and Krawinkler $(2009,2011)$. In the same figure, a third option is also shown (noted as Option 3) that is essentially a modified backbone curve that is penalized for cyclic 
deterioration. Both models have been proposed in recent modeling recommendations presented in PEER/ATC-72-1.

The Ramberg-Osgood model (1943) has been used extensively since the 1960s, and is still used if the Bauschinger effect is deemed important. The original RambergOsgood model is a non-degrading smooth model. However, Albenesi and Nuti (2007) summarize a number of modifications to the hysteretic response of the MenegottoPinto model in order to capture component deterioration. A cyclic hardening factor can be applied to the unloading/reloading curve, but its value should be history dependent. Hardening parameters as well as strength and stiffness degradation parameters are implemented in the Ruaumoko analysis platform (Carr, 2011), which also includes a large list of other hysteretic models.

Ibarra et al. (2005) developed a deterioration model that is able to simulate up to four modes of cyclic deterioration of steel components. The hysteretic rules of this model are based on previous work by Rahnama and Krawinkler (1993). The phenomenological Ibarra-Krawinkler (IK) model is based on a backbone curve that defines a boundary for the behavior of a structural component and establishes strength and deformation bounds, and a set of rules that define the basic characteristics of the hysteretic behavior between the bounds. Lignos and Krawinkler $(2007,2009,2011)$ modified the Ibarra-Krawinkler (IK) model in order to account for asymmetric hysteretic response due to slab effects, a residual strength path, and an ultimate rotation capacity that the component is able to fracture. In the modified IK model cyclic deterioration is quantified using a deterioration parameter that is based on a reference energy dissipation capacity of the component defined as $E_{t}=\Lambda \theta_{p}$, where $\Lambda$ is a property of the component and $\theta_{p}$ is its plastic rotation capacity.

Lignos and Krawinkler $(2009,2011)$ calibrated the hysteretic response of the modified IK model with more than 300 deduced moment rotation relationships of plastic regions in steel beams parts of steel beam-to-column subassemblies. Based on information deduced from the steel component database, empirical relationships for modeling of pre-capping plastic rotation, post-capping rotation and cyclic deterioration for beams with reduced beam section (RBS) and beams other than RBS are proposed. Quantitative information is also provided for modeling of the effective yield strength, post-yield strength ratio, residual strength, and ductile tearing of steel components subjected to cyclic loading. This information has been adopted in PEER/ATC-72-1. More recently, Lignos et al. (2011c) incorporated a low cycle fatigue rule in the modified IK model to investigate the effect of long duration records on the seismic capacity of steel buildings and validated this model with full scale tests of a steel structure representing a typical 20-story steel building designed in Japan in the 1970s. Table A-1 presents a summary of the capabilities of hysteretic models that were reviewed. 
Table A-1 Summary of Capabilities of Hysteretic Models (Lignos and Krawinkler 2009)

\begin{tabular}{|c|c|c|c|c|c|c|c|c|}
\hline \multirow[b]{2}{*}{ Model } & \multirow[b]{2}{*}{$\begin{array}{c}\text { Cyclic } \\
\text { Hardening } \\
\end{array}$} & \multirow[b]{2}{*}{$\begin{array}{c}\text { Bausch } \\
\text { Effect } \\
\end{array}$} & \multirow[b]{2}{*}{$\begin{array}{l}\text { Strength } \\
\text { Capping } \\
\end{array}$} & \multirow[b]{2}{*}{$\begin{array}{l}\text { Residual } \\
\text { Strength }\end{array}$} & \multicolumn{4}{|c|}{ Cyclic Deterioration } \\
\hline & & & & & $\begin{array}{c}\text { Basic } \\
\text { Strength } \\
\end{array}$ & $\begin{array}{l}\text { Post Cap } \\
\text { Strength } \\
\end{array}$ & $\begin{array}{l}\text { Unloading } \\
\text { Stiffness } \\
\end{array}$ & $\begin{array}{c}\text { Accel. } \\
\text { Stiffness }\end{array}$ \\
\hline $\begin{array}{l}\text { Clough and Johnston } \\
\text { with Modifications }\end{array}$ & $x$ & $x$ & $x$ & $x$ & $x$ & $x$ & 0 & 0 \\
\hline Takeda Model & $x$ & $\mathrm{x}$ & $\mathrm{X}$ & $\mathrm{X}$ & $X$ & $x$ & 0 & 0 \\
\hline $\begin{array}{l}\text { Bouc-Wen with } \\
\text { modifications }\end{array}$ & 0 & 0 & $x$ & $x$ & 0 & $x$ & 0 & 0 \\
\hline Ramberg-Osgood & 0 & 0 & $x$ & $x$ & 0 & $x$ & 0 & $x$ \\
\hline Kunath et al. & 0 & 0 & $x$ & $\mathrm{X}$ & 0 & $\mathrm{X}$ & 0 & 0 \\
\hline Syvaselvan and Reinhorn & 0 & 0 & $X$ & $\mathrm{X}$ & 0 & $\mathrm{X}$ & 0 & $X$ \\
\hline ASCE/SEI 41-06 & 0 & $x$ & 0 & 0 & $\mathrm{X}$ & $x$ & $X$ & $X$ \\
\hline $\begin{array}{l}\text { Yield-Line Plastic Hinge } \\
\text { Models }\end{array}$ & 0 & 0 & 0 & 0 & 0 & 0 & 0 & 0 \\
\hline Song-Pincheira & $x$ & $\mathrm{X}$ & 0 & 0 & $X$ & $x$ & 0 & 0 \\
\hline Ibarra-Krawinkler & $x$ & $x$ & 0 & 0 & 0 & 0 & 0 & 0 \\
\hline Modified Ibarra-Krawinkler & $\mathrm{X}$ & $\mathrm{x}$ & 0 & 0 & 0 & 0 & 0 & 0 \\
\hline
\end{tabular}

\section{A.5.1.2 Fiber Models}

At the member cross section, spread of plasticity across the finite region of the plastic hinge length can be idealized as a nonlinear moment-curvature response. Examples of fiber element implementations include Alvarez and Birnstiel (1969), El-Zanaty et al. (1980), Clarke (1994), and Alemdar and White (2005). Kunnath et al. (1990) proposed a new deterioration model, which was based on distributed plasticity concepts. This model is both an extension and a simplification of the Takizawa (1976) model. The Kunnath et al. (1990) model has been incorporated in recent versions of IDARC (Reinhorn et al. 2009). In concept, the model is both simple and versatile since it is based on integration of the curvature diagram that results in the basic incremental moment-rotation relationship. These integrals, which eventually result in the element stiffness matrix, can be obtained in closed form and directly used in a computer program. Kunnath et al. (1990) showed that shear deformations can be directly incorporated. Improved versions of such models have also been investigated. A nonlinear distribution was developed for tapered elements (Kunnath et al., 1992) and a multi-linear distribution with non-symmetric properties was developed for the three-dimensional model (Lobo, 1994).

While the basic formulations for fiber element methods are fairly straightforward, their direct implementation can require excessive computational time and equipment. 
For this reason, a number of researchers have developed models that have the computational efficiency of the concentrated plasticity models and the accuracy of spread-of-plasticity models (Liew et al., 1993; Attalla et al., 1994; El-Tawil and Deierleien, 1996). Nukala and White (2004) have extended these models to include three-dimentional stability behavior.

Flexibility based (Neuenhofer and Fillipou, 1997) or mixed formulation (Spacone et al., 1996; Nukala and White, 2004) fiber models have also been developed. These models better represent the nonlinear curvature fields in a yielded member as compared to displacement-based elements.

More recently, Krishnan (2010) developed an efficient beam element, the modified elastofiber (MEF) that captures the overall features of the elastic and inelastic responses of slender columns and braces under axial cyclic loading without unduly heavy discretization. It consists of three fiber segments, two at the member ends and one at midspan, with two elastic segments sandwiched in between. All the control points on the stress-strain law are user-defined. The elastic buckling of a member is tracked by updating both exterior and interior nodal coordinates at each iteration of a time step and checking force equilibrium in the updated configuration. Inelastic postbuckling response is captured by fiber yielding, fracturing, or rupturing in the nonlinear segments. Key features of the element include the ability to model each member using a single element, easy incorporation of geometric imperfection, partial fixity support conditions, member susceptibility to fracture defined in a probabilistic manner, and fiber rupture leading to complete severing of the member. The model is implemented in the FRAME3D (Krishnan, 2003) analysis software.

\section{A.5.2 Structural System Performance}

A number of investigators have conducted nonlinear response history analyses of steel moment resisting frames (MacRae et al., 1990; Gupta and Krawinkler, 1999, 2000; Medina and Krawinkler, 2003; Ibarra and Krawinkler, 2005; Zareian and Krawinkler, 2007, Luco and Cornell, 2000, Lee and Foutch, 2000, 2002a; Lignos and Krawinkler, 2009). As part of the SAC project, Luco and Cornell (2000) and Lee and Foutch (2002b) assessed the effects of brittle connection fractures on the behavior of archetype steel moment resisting frame structures ranging in height from 2- to 20stories, with pre-Northridge connections, at various levels of ground motion intensity. The frames were designed in three different regions (Los Angeles, Seattle, and Boston). An empirical analysis model (Foutch and Shi, 1996; Lee and Foutch, 2000) was employed for connection fracture, with values of the parameters for the model calibrated by field and laboratory data. In mild ground motions (e.g., 10\% in 50 year ground motions), the effects of flange connection fractures on story drift responses were minimal because the demands were not large enough to induce more than a few fractures. Under moderate ground motions (e.g., $2 \%$ in 50 year ground motions), flange connection fractures had a relatively small effect on median drift demands. 
When subjected to rogue earthquake records, namely, those records that cause relatively large story drifts in the ductile case (e.g., a subset of the $2 \% / 50$ year ground motions), model structures with brittle bottom flange connections experienced extreme story drifts (larger than 10\%), including collapses. It should be pointed out that these assessments were not done with deteriorating models. Luco and Cornell (2000) concluded that extreme drifts such as those encountered under the rogue ground motions (for example) are typically reduced when the contributions of interior gravity frames and shear connections are added to perimeter moment resisting frame model structures.

As part of the SAC Phase II project, Gupta and Krawinkler (2000) assessed the seismic demands of the same archetype frames that were analyzed by Luco and Cornell (2000). The only difference was that the moment resisting frames had ductile connections (i.e., no deterioration and fracture). Gupta and Krawinkler illustrated that modeling assumptions (e.g., centerline bare frame, frame with deformable panel zones, frame with panel zones, and gravity frames) can significantly affect the structural response for cases in which a change in mechanism occurs or when a structure is driven into the range of negative post-yield stiffness.

The distribution of inelastic deformation demands between the elements at a beam-tocolumn connection is sensitive to subjective design decisions and material strength. The expectation that beams attract most of the plastic deformation demands does not hold true if weak panel zones exist. The formation of plastic hinges in the columns of 9-story and 20-story Los Angeles and Seattle structures was observed for many ground motions, particularly in cases where adjacent stories exhibited significantly different story drifts. The presence of large bending moment demands in the columns, together with high axial loads due to large overturning moments, necessitates more detailed research into the behavior of columns and column splices. Considering the distinct potential for column plastic hinging, it is essential to use only compact columns with proper lateral bracing. Structure P-delta effects may greatly influence the seismic response if the ground motion drives the structure into the negative postyield stiffness range in any one story. If this possibility exists, the response can become very sensitive to modeling assumptions and structure and ground-motion characteristics. This issue is discussed in a companion paper (Gupta and Krawinkler, 2000).

The effect of component deterioration on the seismic performance of moment resisting frames with post-Northridge connections designed as part of the SAC program was assessed by Lee and Foutch (2000). A component model was used to simulate cyclic deterioration of the connections. However, in this model the postcapping strength deterioration was not modeled. It has been shown both analytically (Ibarra and Krawinkler, 2005; Zareian and Krawinkler, 2007; Lignos and Krawinkler, 2009) and experimentally (Lignos et al., 2010, 2011d; Suita et al., 2008) that the 
capping point is critical for assessing the collapse potential of moment resisting frames (Ibarra and Krawinkler, 2005; Lignos and Krawinkler, 2009; Lignos et al., 2011a). Lee and Foutch (2002a) concluded that all of the buildings with postNorthridge connections showed a high capacity-to-demand ratio ranging from 2.0 to 4.0, depending on frame configuration and height. As a result, it was decided that post-Northridge connections should exhibit good performance in future seismic events.

\section{A.5.2.1 Collapse Assessment of Moment Resisting Frames}

The collapse potential of moment resisting frames is assessed through the use of collapse fragility functions (Ibarra and Krawinkler, 2002, 2005; Medina and Krawinkler, 2003; Zareian and Krawinkler, 2007). FEMA P-695, Quantification of Building Seismic Performance Factors (FEMA 2009b), provides a methodology for quantifying building system performance in the context of collapse safety. In this methodology, the safety margin against collapse of a building designed with a specific response modification coefficient ( $R$ factor) is quantified and compared with an acceptable safety margin that is chosen based on a defined probability of collapse and an assumed level of variability.

In the FEMA P-695 methodology, design provisions are gathered and substantiated by component test information and professional design experience. Structures are designed that are representative of the current building stock and in conformance with the design provisions, termed "archetypes." Analytical models of these archetypes are developed using state-of-the-art modeling techniques that take advantage of experimental data. By incorporating uncertainty considering quality of modeling, design requirements, and test data, and by utilizing nonlinear response history analysis for a prescribed set of ground motions, the required safety margin against collapse for each archetype model is calculated. FEMA P-695 acceptance criteria are twofold: (1) less than 20\% probability of collapse for individual archetypes, given the Maximum Considered Earthquake (MCE) hazard level; and (2) less than 10\% probability of collapse on average across all archetypes, given the MCE hazard level. If the criteria are not met, the response modification coefficient, $R$, should be modified and the archetypes should be re-designed. The iterative loop continues until the proposed seismic performance factors provide adequate collapse safety.

NIST GCR 10-917-8, Evaluation of the FEMA P-695 Methodology for Quantification of Building Seismic Performance Factors (NIST, 2010), assessed the collapse potential of 1- to 20-story archetype buildings with perimeter 3-bay moment resisting frames designed in accordance with ASCE/SEI 7-05 (ASCE, 2007), except that $C_{d}=R=8$ (per FEMA P-695 requirements). Archetype configurations used in this study covered the design space with variations in seismic design categories (e.g., SDC $D_{\max }$, and SDC $D_{\min }$ ), and design procedure (e.g., ELF, and RSA). All connections 
were RBS connections designed in accordance with ANSI/AISC 358-05 (AISC, $2005 \mathrm{~b}$ ), and the effect of gravity framing was not included in the analytical models.

The main outcome of these studies was that the long-period designs in the SDC $D_{\max }$ region did not pass the acceptability criteria. For these long period structures, amplification of story drifts in the lower stories, caused by P-Delta effects, dominated the response in the inelastic range and led to development of a partial mechanism that involved the lower few stories of the structural system (Lignos et al., 2011a; Zareian et al., 2011).

In the end columns of the frames, axial load ratios ranged from 0.30 to 0.80 due to dynamic axial loads from global overturning moments. In this study deep columns ranging from W24 to heavy W36 were used. In a follow-up study with the same moment resisting frames, Zareian et al. (2011) showed that by increasing the strongcolumn-weak-beam (SCWB) strength ratio from unity to 1.2, archetypes passed the collapse safety criteria. The improved collapse safety was due to a change in the collapse mechanism of the structural system, whereby an increase in the SCWB ratio distributed the story drift and inelastic deformation demands over a larger number of stories.

\section{A.6 Common Failure Modes in Deep, Slender Wide Flange Structural Steel Beam-Column Members}

A list of critical failure modes in deep, slender wide-flange structural steel beamcolumn members is summarized below. Each can contribute to collapse or partial collapse of steel moment resisting frames, as indicated by evidence from past earthquakes. The order listed does not imply a level of importance or frequency.

\section{A.6.1 Global Failure Mechanisms}

Flexural buckling. Flexural buckling occurs when the axial load developed in a column attains the Euler load and the column buckles in a flexural mode. Figure A-12 illustrates an example of this failure mode. Due to presence of residual stresses and out-of-plane straightness, bifurcation buckling occurs and the maximum load approaches the Euler load asymptotically, as long as the steel remains elastic. This mode is the dominant failure mode that most standard hot-rolled shapes with doubly symmetric cross sections experience.

Lateral-torsional buckling. Wide flange sections subjected to flexure typically have much greater strength and stiffness about their major principal axis than their minor axis. Unless these members are properly braced against lateral deflection and twisting, they are subject to lateral-torsional buckling prior to the attainment of their full in-plane bending capacity. Wide flange sections are especially prone to this type of buckling during the construction phase, when braces are either temporary or absent. Lateral-torsional buckling is a limit state where the deformation changes from 
predominantly in-plane bending to combined lateral deflection and twisting. The final failure pattern involves lateral deflection and twisting in combination with some extent of yielding and flange or web local buckling, depending on the characteristics of the cross section.

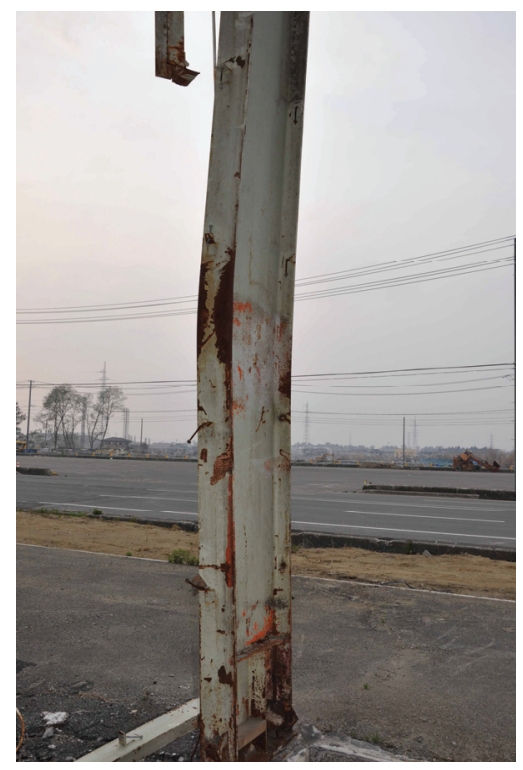

Figure A-12 Flexural buckling of a steel wide flange column (courtesy of D. Lignos).

Torsional or flexural-torsional buckling. To prevent buckling about the weak axis, wide flange columns are frequently restrained in the lateral direction. The buckling mode is then defined by a rotation of the cross-section about the axis of lateral restraint. Depending on the location of the restraints, the column may react in a torsional deformation, or in a combined torsional and flexural deformation. An example of this failure mode is illustrated in Figure A-13 from a near full-scale test on a retrofitted steel moment frame (Lignos et al., 2011f).

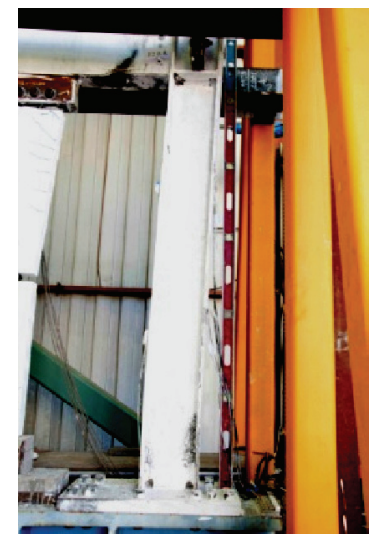

(a) column behavior

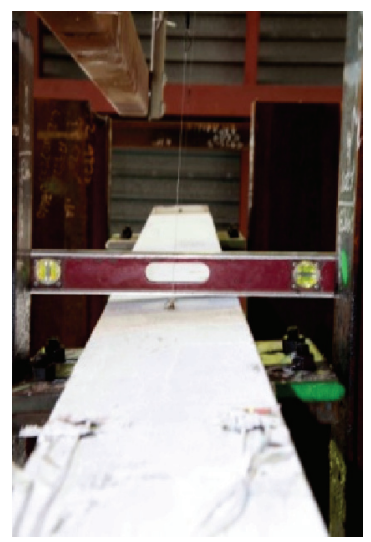

(b) torsional deformation

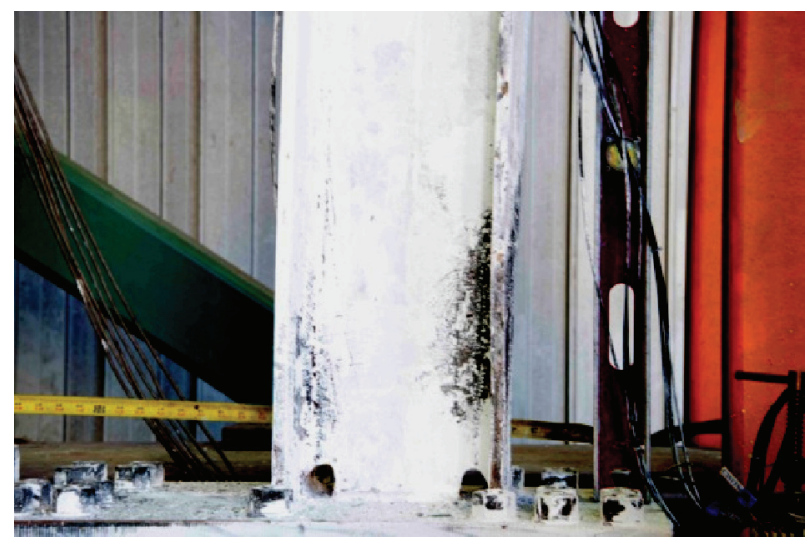

(c) torsional and flexural deformation

Figure A-13 Torsional-flexural buckling of a steel wide flange column (courtesy of D. Lignos). 
Local buckling at plastic hinges. The consequence of local buckling in conjunction with the development of a plastic hinge in a steel column results in strength deterioration of its flexural capacity. For slender steel columns, the influence of axial loads applied to columns subjected to cyclic loading can be detrimental (Popov et al., 1975) since the negative stiffness after formation of a plastic hinge can be steep and can lead to collapse.

Figure A-14a shows plastic hinge formation in the column of a subassembly that has been subjected to high axial forces and shear reversals simulating the effect of seismic loading (Popov et al., 1975). Figure A-14b shows severe local buckling in the flanges and web at the bottom of the first story of the south-west corner column of the Cordova building in Anchorage, Alaska following the 1967 earthquake. Figure A-15c shows the plastic hinge formation at the base of a first story column in an E-Defense shake table test of a full-scale, 4-story moment resisting frame with square hollow tubular columns (Suita et al., 2008).

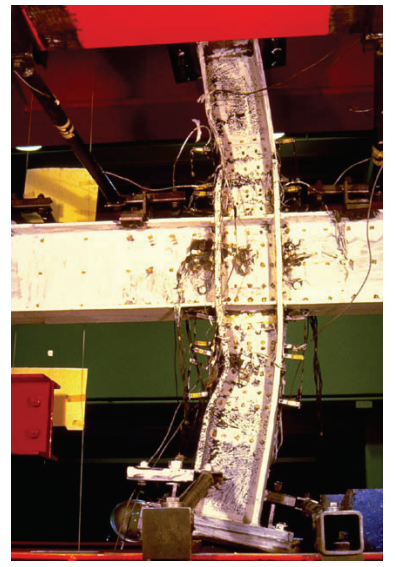

(a)

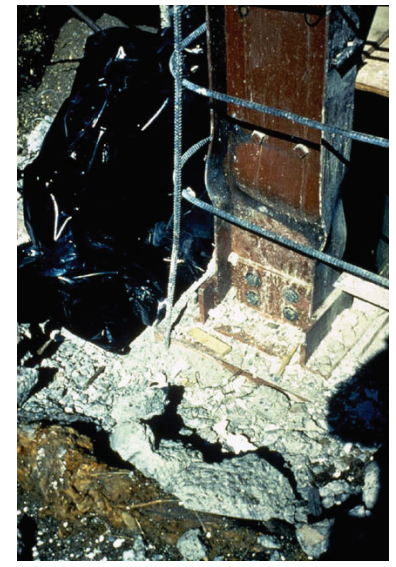

(b)

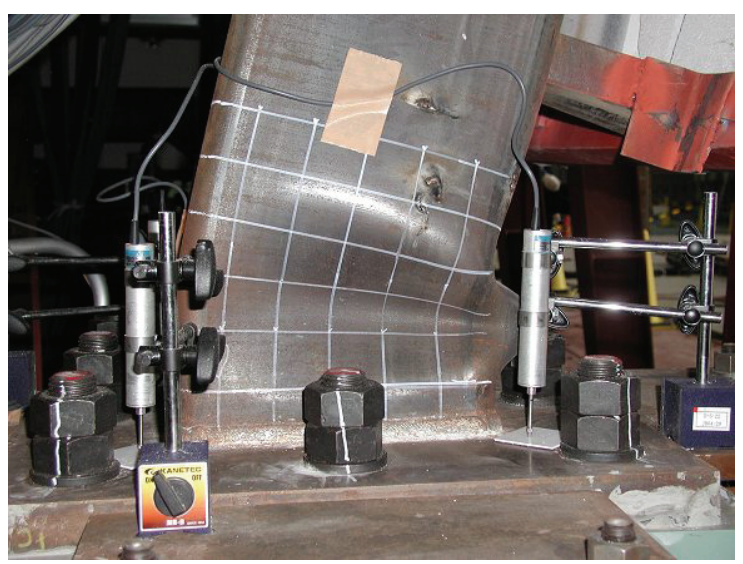

(c)

Figure A-14 Buckling of steel columns with plastic hinges (courtesy of NISEE, PEER, and $\mathrm{H}$. Krawinkler).

Dynamic instability. Steel moment resisting frames can experience large lateral deformations during severe earthquakes. These can lead to significant additional overturning moments due to gravity loads acting on the laterally deformed structure. Second order P-Delta effects, accelerated by component deterioration, can fully overcome first order story shear resistance, resulting in dynamic instability. Figure A-16 illustrates global instability in a recent full-scale collapse test conducted in Japan.

\section{A.6.2 Local Buckling of Plate Elements}

Unlike a single plate element supported along unloaded edges, a plate assembly such as a wide flange can buckle in different ways. In the case of axial compression, instability can occur in of one of three buckling modes: 
Local Buckling (Mode I): A pure local buckling mode involves out-of-plane deformation of the constituent plates, with the points of intersection remaining essentially straight. Associated half-wavelengths are the same order of magnitude as the widths of the plate elements.

Distortional Buckling (Mode II): Distortional buckling can involve in-plane bending of one or more of the constituent plates along with out-of-plane bending of all the elements in a purely local mode. This buckling mode is also referred to as a stiffener buckling mode, local torsional mode, or orthotropic mode, depending on the context. Associated wavelengths are considerably greater than those of local buckling (Mode I), but there is a half-wavelength at which the critical stress is a minimum.

Global Buckling (Mode III): The plate structure can buckle as a column in flexural or flexural-torsional mode with or without the interaction of local buckling. Finite strip analysis has become a popular alternative to shell finite element analysis for this problem, with several programs available for this purpose (Hancock, 1978; Papangelis and Hancock, 1995; Schafer and Adany, 2006). Another analysis option seeing significant attention is generalized beam theory (Silvestre and Camotim, 2002).

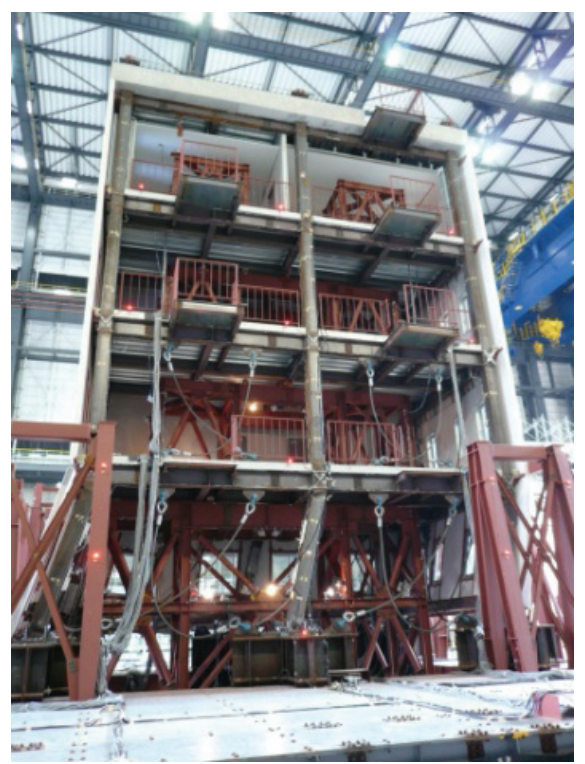

Figure A-15 Collapse due to dynamic instability (courtesy of D. Lignos).

More complete information on $K$-factors as influenced by the interaction between plate components can be found in several references (Bleich, 1952; Stowell et al., 1952; Gerard and Becker, 1957; Timoshenko and Gere, 1961). Such calculations, however, must be used with caution because: (1) results can be unduly conservative when the plate structure consists of elements with widely varying slenderness due to neglect of the rotational restraint at the junctions; and (2) results are inapplicable unless it is ensured that all the plate elements buckle locally (i.e., the junctions remain essentially straight). If, on the other hand, distortional or global buckling (Modes II or III) are critical, such simplified calculations overpredict strength. 
Distortional or stiffener buckling (Mode II) may be averted in practice by providing intermediate or edge stiffeners of adequate rigidity. This can be advantageous because of the limited post-buckling resistance associated with Mode II buckling, but is not always practical or economical. Hence, distortional buckling must often be considered in design. Variations in the local buckling coefficients $k_{w}$ for a wideflange I-section with respect to the geometrical properties of the shape have been reported in charts (Zeimian 2010). Additional charts and related information can be found in references such as the Handbook of Structural Stability (Column Research Committee of Japan, 1971). In addition, free or low-cost computational tools (Papangelis and Hancock, 1995; Schafer and Adany, 2006; Silvestre and Camotim, 2002) are available and provide similar results.

Interaction between the elements of a plate assembly exists because of equilibrium and compatibility conditions that must be satisfied at the junctions. In the case of local buckling it is possible to simplify these conditions considerably, as shown by Benthem (1959). Because in-plane displacements are much smaller than out-of-plane displacements, it is possible to assume that normal displacements are zero for each plate element meeting at a corner.

In the post-buckling range, in-plane displacements and membrane stresses dominate the behavior of the buckled plates. The interactions between plate elements along the junctions become very complex. The problem is compounded when interaction between global and local buckling is considered, or when interaction between distortional and the other modes is considered. For these reasons, post-buckling and strength of plate assemblies considering all possible instabilities (local, distortional, and global) remains a topic of considerable research. Earlier references on interaction of local and overall instability in the post-buckling range include those by Bijlaard and Fisher (1952), Cherry (1960), Graves-Smith (1969) and Skaloud and Zornerova (1970). Research in this field has also been carried out by Thomasson (1978), Little (1979), Hancock (1980, 1981) and Bradford and Hancock (1984). Using the concept of effective width, the problem has also been investigated by DeWolf et al. (1974) and Desmond et al. (1981a, 1981b).

As design methods for the combination of local and global buckling mature, recent research has focused attention on distortional buckling. Lau and Hancock (1987) provided an analytical formula for predicting elastic distortional buckling, and later Hancock et al. (1994) provided empirical formulas for predicting the ultimate strength in distortional buckling for a variety of different cold-formed steel cross sections. Bradford (1992) examined the distortional mode in the context of hot-rolled steel Ibeams, while Schafer (2002) and Schafer and Pekoz (1999) examined local, distortional, and global buckling of cold-formed steel beams and columns in developing the methods currently used in AISI-S100-07, Specification for the Design of Cold-Formed Steel Structural Members (AISI, 2007). 


\section{A.7 Summary and Limitations of Design Criteria for Deep, Slender Wide-Flange Structural Steel Beam-Column Members}

In ANSI/AISC 341-10 seismic provisions, all columns in special moment frame (SMF) systems must meet stringent seismic compactness requirements, even if a strong column design is enforced. Less stringent limits for compact sections in the ANSI/AISC 360-10 specification apply to intermediate moment frame (IMF) systems.

In CAN/CSA-S16, Limit States Design of Steel Construction (CSA, 2001), the most severe cross-sectional slenderness limits apply for both type D (ductile) and type MD (moderately ductile) moment resisting frames, but only to columns where plastic hinging is expected. Axial loads are also restricted in these columns to $0.3 P_{y}$ and $0.5 P_{y}$ for types $\mathrm{D}$ and $\mathrm{MD}$ frames, respectively. Less severe compactness limits can be used for columns designed to remain elastic.

In NZ 3404, Steel Structures Standard (NZS, 2007), two limits are imposed on design column axial loads in addition to cross-section slenderness requirements. One is related to the anticipated inelastic demand (ductility category), defined by $0.5 P_{y}$ to $1.0 P_{y}$ for the most ductile to the less ductile systems. The second limit depends on column slenderness and aims to avoid plastic hinges forming between column ends. NZ 3404 also limits column axial loads due to gravity loads acting alone, and this limit is expressed as a function of the column web slenderness ratio. For fixed-base columns, strength degradation at connections to the foundation can be prevented by designing column base plates for the full flexural strength of the columns. This is explicitly required in ANSI/AISC 341-10 and CAN/CSA-S16 seismic provisions, and details to achieve this behavior can be found in Arlekar and Murty (2002) and Fahmy et al. (2000).

Depending on the beam-to-column connection typology, additional local stability verifications may be needed in the joint region of the columns. For instance, the column web must be checked for web crippling, web buckling in compression, and web buckling in shear for joints in which the beams are directly welded to wide flange columns bent about their major axis. Design guidance for these cases can be found in Steel Design Guide 13: Stiffening of Wide-Flange Columns at Moment Connections: Wind and Seismic Applications (AISC, 2003) and Eurocode 3 (CEN, 2002). Web crippling and compression web buckling are verified for unstiffened connections (without continuity plates or transverse stiffeners). Limits are prescribed to prevent stability failure of webs acting in shear. These limits are usually satisfied for the majority of hot-rolled wide flange sections used in columns, but they could control in cases where built-up columns with relatively thin webs or higher grades of steel are used. 



\section{References}

ACI, 2006, Code Requirements for Nuclear Safety Related Concrete Structures and Commentary, ACI 349-06/349R-06, American Concrete Institute Committee 349, Farmington Hills, Michigan.

AISC, 1986, Load and Resistance Factor Design Specification for Structural Steel

Buildings, American Institute of Steel Construction, Chicago, Illinois.

AISC, 1990, Steel Design Guide 1: Column Base Plates, American Institute of Steel Construction, Chicago, Illinois.

AISC, 2003, Steel Design Guide 13: Stiffening of Wide-Flange Columns at Moment Connections: Wind and Seismic Applications, $2^{\text {nd }}$ printing, American Institute of Steel Construction, Chicago, Illinois.

AISC, 2005a, Manual of Steel Construction, $13^{\text {th }}$ Edition, American Institute of Steel Construction, Chicago, Illinois.

AISC, 2005b, Prequalified Connections for Special and Intermediate Steel Moment

Frames for Seismic Applications, ANSI/AISC 358-05, American Institute of Steel Construction, Chicago, Illinois.

AISC, 2006, Steel Design Guide 1: Base Plate and Anchor Rod Design, Second

Edition, American Institute of Steel Construction, Chicago, Illinois.

AISC, 2010a, Seismic Provisions for Structural Steel Buildings, ANSI/AISC 341-10, American Institute of Steel Construction, Chicago, Illinois.

AISC, 2010b, Prequalified Connections for Special and Intermediate Steel Moment Frames for Seismic Applications, ANSI/AISC 358-10, American Institute of Steel Construction, Chicago, Illinois.

AISC, 2010c, Specification for Structural Steel Buildings, ANSI/AISC 360-10,

American Institute of Steel Construction, Chicago, Illinois.

AISI, 2007, Specification for the Design of Cold-Formed Steel Structural Members, AISI-S100-07, American Iron and Steel Institute, Washington, D.C.

Albenesi, T. and Nuti C., 2007, Reinforcing Steel Bar Model, Dispensa, Universita Degli Studi, ROMA TRE, Dipartimento di Strutture. 
Alemdar, B.N. and White, D.W., 2005, "Displacement, flexibility and mixed beamcolumn finite element formulations for distributed plasticity analysis," Journal of Structural Engineering, Vol. 131, No. 12, pp. 1811-1819.

Alpsten, G.A., 1972, "Prediction of thermal residual stresses in hot rolled plates and shapes," Final Report, $9^{\text {th }}$ Congr. IABSE, Final Rep., Amsterdam, May, pp. 1-13.

Alpsten, G.A. and Tall, L., 1970, "Residual stresses in heavy welded shapes," the Welding Journal, Vol. 49, No. 3, pp. 93-105.

Alvarez, R.J. and Birnstiel, C., 1969, "Inelastic analysis of multistory multibay frames," Journal of the Structural Division, Vol. 95, No. ST11, pp. 2477 2503.

Anastasiadis, A., Gioncu, V., and Mazzolani, F.M., 2000, "New trends in the evaluation of available ductility of steel members," in Behavior and Ductility of Steel Structures, eds. F.M. Mazzolani and R. Tremblay, Taylor \& Francis, New York, New York.

Anslijn, R., 1983, Tests on Steel I Beam-Columns in Mild Steel Subjected to Thrust and Biaxial Bending, CRIF Rep. MT 157, Brussels, Belgium.

ANSYS, 2009, “Command reference," Release 12, ANSYS, Inc., Canonsburg, Pennsylvania.

Arbona, F.J.D., Castro, J.M., Elghazouli, A.Y., 2008, Assessment of panel zone design approaches for steel moment frames," Proceedings, $14^{\text {th }}$ World Conference on Earthquake Engineering, October 12-17, 2008, Beijing, China.

Architectural Institute of Japan (AIJ), 2006, Report of Seismic Performance Improvement of Civil, Architectural Structures Subjected to Long-Period Ground Motions Generated by Subduction Zone, Japan Society of Civil Engineering, Tokyo (in Japanese).

Arlekar, J.N., and Murty, C.V.R., 2002, "P-V-M Interaction curves for seismic design of steel column base connections," Engineering Journal, Vol. 39, No. 3, pp. 154-165.

ASCE, 2006, Minimum Design Loads for Buildings and Other Structures, ASCE/SEI 7-05, American Society of Civil Engineers, Reston, Virginia.

ASCE, 2007, Seismic Rehabilitation of Existing Buildings, ASCE/SEI 41-06, American Society of Civil Engineers, Reston, Virginia.

ASCE, 2010, Minimum Designs Loads for Buildings and Other Structures, ASCE/SEI 7-10, American Society of Civil Engineers, Reston, Virginia. 
Assadi, M. and Roeder, C.W., 1985, "Lateral buckling of continuously restrained cantilevers," Journal of Engineering Mechanics, Vol. III, No. 12, pp. 14401456.

Astaneh, A., Bergsma, G., and Shen J.H., 1992, "Behavior and design of base plates for gravity, wind and seismic loads," Proceedings, National Steel Construction Conference, Las Vegas, Nevada.

Astaneh, A. and Ravat, S., 1998, Cyclic Behavior and Seismic Design of Steel HPiles, Report UCB/CEE-Steel-98/01, Department of Civil and Environmental Engineering, University of California, Berkeley, California.

ATC, 2003, The Missing Piece: Improving Seismic Design and Construction Practices, ATC-57 Report, Applied Technology Council, Redwood City, California.

Attalla, M.R., Deierlein, G.G., and McGuire, W., 1994, "Spread of plasticity: a quasiplastic hinge approach," Journal of Structural Engineering, Vol. 120, No. 8, pp. 2451-2473.

Baber, T. and Noori, M.N., 1985, "Random vibration of degrading, pinching systems," Journal of Engineering Mechanics, Vol. 111, No. 8, pp. 10101026.

Baber, T. and Noori, M.N., 1986, "Modeling general hysteresis behavior and random vibration application," Journal of Vibration, Acoustics, Stress and Reliability in Design, Vol. 108, pp. 411-420.

Ballio, G., Petrini, V., and Urbano, C., 1973, "The effect of the loading process and imperfections on the load bearing capacity of beam columns," Meccanica, Vol. 8, No. 1. pp. 56-67.

Ballio, G. and Campanini, C., 1981, "Equivalent bending moments for beamcolumns," Journal of Constructional Steel Research, Vol. 1, No. 3.

Balut, N. and Moldovan, A., 1997, "A model for the behavior of column base connections," Proceedings, Second International Conference on the Behavior of Steel Structures in Seismic Areas, STESSA ‘97, Kyoto, Japan.

Batterman, R.H. and Johnston, B.G., 1967, "Behavior and maximum strength of metal columns," Journal of the Structural Division, Vol. 93, No. ST2, pp. 205-230.

Becker, E.R., 1971, Panel Zone Effect on the Strength and Stiffness of Rigid Steel Frames, Research report, Mechanical Lab., University of Southern California, Los Angeles, California.

Beedle, L.S. and Tall, L., 1960, "Basic column strength," Journal of the Structural Division, Vol. 86, No. ST5, pp. 139-173. 
Beedle, L.S., ed., 1993, Semi-rigid Connections in Steel Frames, Council on Tall Buildings and Urban Habitat, McGraw-Hill, New York.

Benthem, J.P., 1959, The Reduction in Stiffness of Combinations of Rectangular Plates in Compression After Exceeding the Buckling Load, NLL-TR S-539.

Bertero, V.V., Krawinkler, H., Popov, E.P., 1973, Further Studies on Seismic Behavior of Steel Beam-to-Column Subassemblies, Report EERC-73-27, University of California, Berkeley, California.

Bijlaard, P.P. and Fisher, G.P., 1952, Column Strength of H Sections and Square Tubes, in Post Buckling Range of Component Plates, NACA Tech. Note No. 2994, National Advisory Committee for Aeronautics, Washington, D.C.

Bijlaard, P.P., Fisher, G.P., and Winter, G., 1955, "Eccentrically loaded, endrestrained columns," Transactions of the American Society of Civil Engineering, Vol. 120, p. 1070.

Birnstiel, C., 1968, "Experiments on H-columns under biaxial bending," Journal of the Structural Division, Vol. 94, No. ST10, pp. 2429-2450.

Bouc, R., 1967, "Forced vibration of mechanical systems with hysteresis," Abstract Proceedings, $4^{\text {th }}$ Conference on Nonlinear Oscillation, Prague, Czechoslovakia.

Bjorhovde, R., 1972, Deterministic and Probabilistic Approaches to the Strength of Steel Columns, Ph.D. dissertation, Lehigh University, Bethlehem, Pennsylvania.

Bjorhovde, R. and Tall, L., 1971, Maximum Column Strength and the Multiple Column Curve Concept, Fritz Engineering Laboratory, Rep. No. 338.29, Lehigh University, Bethlehem, Pennsylvania.

Bleich, F., 1952, Buckling Strength of Metal Structures, McGraw-Hill, New York.

Bradford, M.A. and Hancock, G.J., 1984, "Elastic interaction of local and lateral buckling in beams," Thin-Walled Structures, Vol. 2, No. 1, pp. 1-25.

Bradford, M.A. and Trahair, N.S., 1985, "Inelastic buckling of beam-columns with unequal end-moments," Journal of Constructional Steel Research, Vol. 5, No. 3, pp. 195-212.

Bradford, M.A. and Trahair, N.S., 1986, "Inelastic buckling tests of beam-columns," Journal of Structural Engineering, Vol. 112, No. 3, pp. 538-549.

Bradford, M.A., 1992, "Lateral-distortional buckling of steel i-section members," Journal of Constructional Steel Research, Vol. 23, Nos. 1-3, pp. 97-116. 
Brozzetti, J., Alpsten, G.A., and Tall, L., 1970a, Residual Stresses in a Heavy Rolled Shape 14WF730, Fritz Eng. Lab. Rep. No. 337.1, Lehigh University, Bethlehem, Pennsylvania.

Brozzetti, J., Alpsten, G.A., and Tall, L., 1970b, Welding Parameters, Thick Plates and Column Strength, Fritz Eng. Lab. Rep. No. 337.21, Lehigh University, Bethlehem, Pennsylvania.

Brunaeu, M., Mahin, S.A., and Popov, E.P., 1987, Ultimate Behavior of Butt Welded Splices in Heavy Rolled Steel Sections, Report No. UBC/EERC-87/10, University of California, Berkeley, California.

Bruneau, M. and Mahin, S.A., 1991, "Full scale tests of butt welded splices in heavy rolled steel sections subjected to primary tensile stresses," Engineering Journal, Vol. 28, No. 1, pp. 1-17.

Burda, J.J., Itani, A.M., 1999, Studies of Seismic Behavior of Steel Base Plates, Report No. CCEER 99-7, Center of Civil Engineering Earthquake Research, Department of Civil and Environmental Engineering, University of Nevada, Reno, Nevada.

Carr, A.J., 2001, RUAUMOKO, the Maori God of Volcanoes and Earthquakes, Department of Civil Engineering, University of Canterbury, Christchurch, New Zealand.

Castro, J.M., Elghazouli, A.Y., and Izzuddin, B.A., 2005, "Modelling of the panel zone in steel and composite moment frames," Engineering Structures, Vol. 27, pp. 129-144.

Castiglioni, C.A., Bernuzzi, C., and Agatino, M.R., 1998, "Design and damage assessment of structural steel components under seismic actions," Proceedings of the NEHRP Conference and Workshop on Research on the Northridge, California Earthquake of January 17, 1994, California Universities for Research in Earthquake Engineering (CUREe), Berkeley, California.

CEN, 2002, Eurocode 3: Design of Steel Structures -Part 1.8: Design of Joints, prEN 1993-1-8:E, European Committee for Standardization, Brussels, Belgium.

CEN, 2004, Eurocode 8: Design of Structures for Earthquake Resistance-Part 1: General Rules, Seismic Actions and Rules for Buildings, EN 1998-1:2004:E, European Committee for Standardization, Brussels, Belgium.

Chan, H.Y. and Lin, K.C., 2007, "Effects of panel zone modeling in seismic performance evaluation of steel moment-resisting frames," Proceedings, $8^{\text {th }}$ 
Pacific Conference on Earthquake Engineering, December 5-7, 2007, Singapore.

Chapuis, J. and Galambos, T.V., 1982, "Restrained crooked aluminum columns," Journal of the Structural Division, Vol. 108, No. ST3, pp. 511-524.

Charney, F.A. and Downs, W.M., 2004, "Modeling procedures for panel zone deformations in moment resisting frames," Proceedings, Connections in Steel Structures V, Amsterdam, Netherlands.

Chen, W.F., 1970, "General solution of inelastic beam-column problem," Journal of the Engineering Mechanics Division, Vol. 96, No. 4, pp. 421-442.

Chen, W.F., 1971a, "Approximate solution of beam columns," Journal of the Structural Division, Vol. 97, No. ST2, pp. 743-751.

Chen, W.F., 1971b, "Further studies of an inelastic beam-column problem," Journal of the Structural Division, Vol. 97, No. ST2, pp. 529-544.

Chen, W.F. and Atsuta, T., 1972, "Column-curvature curve method for analysis of beam-columns," The Structural Engineer, Institution of Structural Engineers, Vol. 50, No. 6, pp. 233-240.

Chen, W.F., 1980, "End restraint and column stability," Journal of the Structural Division, Vol. 105, No. ST11, pp. 2279-2295.

Chen, W.F., ed., 1987, “Joint flexibility in steel frames," Special Issue, Journal of Constructional Steel Research, Vol. 8.

Chen, W.F., ed., 1988, Steel Beam-to-Column Building Connections, Elsevier Applied Science, London, United Kingdom.

Chen, W.F. and Kishi, N., 1989, "Semi-rigid steel beam-to-column connections: data base and modeling," Journal of Structural Engineering, Vol. 115, No. 1, pp. 105-119.

Chen, W.F. and Lui, E.M., 1991, Stability Design of Steel Frames, CRC Press, Boca Raton, Florida.

Chen, W.F., Goto, Y., and Liew, J.Y.R., 1996, Stability Design of Semi-Rigid Frames, Wiley, New York.

Cherry, S., 1960, "The stability of beams with buckled compression flanges," The Structural Engineer, Vol. 38, No. 9, pp. 277-285.

Choi, J., Goel, S.C., and Stojadinovic, B., 2000, Development of Free Flange Moment Connection, Research Report No. UMCEE 00-15, Deptartment of Civil and Environmental Engineering, University of Michigan, Ann Arbor, Michigan. 
Chubkin, G.M., 1959, "Experimental research on stability of thin plate steel members with biaxial eccentricity," Analysis of Spatial Structures, Vol. 5, Paper 6.

Chung, Y.L., Nagae, T., Hitaka, T., and Nakashima, M., 2010, "Seismic resistance capacity of high-rise buildings subjected to long-period ground motions: edefense shaking table test." Journal of Structural Engineering, Vol. 136, No. 6, pp. 637-644.

Chung, Y., Nagae, T., Matsumiya, T., and Nakashima, M., 2011, "Seismic resistance capacity of beam-column connections in high-rise buildings: E-Defense shaking table test," Earthquake Engineering and Structural Dynamics (available online).

Clark, J.W. and Hill, H.N., 1960, "Lateral buckling of beams," Journal of the Structural Division, Vol. 86, No. ST7, pp. 175-196.

Clark, P., Frank, K., Krawinkler, H., and Shaw, R., 1997, Protocol for Fabrication, Inspection, Testing, and Documentation of Beam-Column Connection Tests and Other Experimental Specimens, SAC Steel Project Background Document, Report No. SAC/BD-97/02, prepared by the SAC Joint Venture, a partnership of the Structural Engineers Association of California (SEAOC), Applied Technology Council (ATC), and California Universities for Research in Earthquake Engineering (CUREe).

Clarke, M., 1994, "Plastic-zone analysis of frames," Advanced Analysis of Steel Frames, eds. W.F. Chen and S. Toma, CRC Press, Boca Raton, Florida.

Coelho, G.A.M. and Bijlaard, F.S.K., 2008, Requirements for the Design of Column Splices, Report No. 6-08-3. Delft University of Technology.

Coelho, G.A.M., Simão, P. D., and Bijlaard F.S.K., 2010, "Stability design criteria for steel column splices," Journal of Constructional Steel Research, Vol. 66, pp. 1261-1277.

Cofie, N.G. and Krawinkler, H., 1985, "Uniaxial cyclic stress-strain behavior of structural steel," Journal of Engineering Mechanics, Vol. 111, No. 9, pp. 1105-1120.

Column Research Committee of Japan (CRCJ), 1971, Handbook of Structural Stability, Corona Publishing Co., Tokyo, Japan.

Cordova, P.P. and Deierlein, G.G., 2005, Validation of the Seismic Performance of Composite RCS Frames: Full-Scale Testing, Analytical Modeling, and Seismic Design, Report No. 155, John A. Blume Earthquake Engineering Research Center, Stanford University, Stanford, California. 
CSA, 2001, Limit States Design of Steel Construction, CAN/CSA-S16.1, Including CSA-S16S1-05 Supplement No. 1, Canadian Standards Association, Willowdale, Ontario, Canada.

Cui, Y., Nagae, T., and Nakashima, M., 2009, "Hysteretic behavior and strength capacity of shallowly embedded steel column bases," Journal of Structural Engineering, Vol. 135, No. 10, pp. 1231-1238.

Culver, C. G., 1966a, "Exact solution of the biaxial bending equations," Journal of the Structural Division, Vol. 92, No. ST2, pp. 63-84.

Culver, C.G., 1966b, "Initial imperfections in biaxial bending," Journal of the Structural Division, Vol. 92, No. ST3, pp. 119-138.

Dafalias, Y.F., 1975, On Cyclic and Anisotropic Plasticity: (I) A General Model Including Material Behavior Under Stress Reversals. (II) Anisotropic Hardening for Initially Orthotropic Materials, Ph.D. Dissertation, Department of Civil Engineering, University of California, Berkeley, California.

Desmond, T.P., Pekoz, T., and Winter, G., 1981a, "Edge stiffeners for thin-walled members," Journal of the Structural Division, Vol. 107, No. ST2, Proc. Pap. 16056, pp. 329-354.

Desmond, T.P., Pekoz, T., and Winter, G., 1981b "Intermediate stiffeners for thin walled members," Journal of the Structural Division, Vol. 107, No. ST4, Proc. Pap. 16194, pp. 627-648.

DeWolf, J.T., 1978, “Axially loaded column base plates," Journal of the Structural Division, Vol. 104, No. ST5, pp. 781-794.

DeWolf, J.T. and Sarisley, E.F., 1980, "Column base plates with axial loads and moments," Journal of the Structural Division, Vol. 106, No. 11, pp. 2167 2184 .

DeWolf, J.T., Pekoz, T., and Winter, G., 1974, "Local and overall buckling of cold formed members," Journal of the Structural Division, Vol. 100, No. ST10, pp. 2017-2306.

Djalaly, H., 1975, "Calcul de la resistance ultime des barres comprimees et flechies," Constr. Met., Vol. 12, No. 4, pp. 17-47.

Drake, R.M. and Elkin, S.J., 1999, "Beam-column base plate design - LRFD method," Engineering Journal, Vol. 36, No. 1, pp. 29-38.

Driver, R.G., Grondin, G.Y., Teh, L., 2003, Centrally Loaded Column Research Inventory, Structural Stability Research Council (SSRC), Rolla, Missouri. 
Duan, L. and Chen, W.F., 1990, "Design interaction equation for cylindrical tubular beam-columns," Journal of Structural Engineering, Vol. 116, No. 7, pp.

1794-1812.

Dux, P.F. and Kitipornchai, S., 1981, Inelastic Beam-Buckling Experiments, Res.

Rep. No. CE24, Department of Civil Engineering, University of Queensland, St. Lucia, Australia.

Dwyer, T.J. and Galambos, T.V., 1965, "Plastic behaviour of tubular beamcolumns," Journal of the Structural Division, Vol. 91, No. ST4, pp. 125-152.

E-Defense Steel Building Project, 2007, Four-story Steel Building Collapse Analysis Blind Prediction, http://www.blind-analysis.jp/index e.html.

Essa, H.S. and Kennedy, D.J.L., 1993, Distortional Buckling of Steel Beams, Struct. Eng. Rep. No. 185, Department of Civil Engineering, University of Alberta, Edmonton, Alberta.

El-Tawil, S., 2000, "Panel zone yielding in steel moment connections," Engineering Journal, Vol. 37, No. 3, pp. 120-135.

El-Tawil, S. and Deierlein, G.G., 1996, "Inelastic analysis of mixed steel-concrete systems," Proceedings, $5^{\text {th }}$ Int. Colloq. Stab. Met. Struct., SSRC, Lehigh University, Bethlehem, Pennsylvania.

El-Tawil, S. and Deierlein, G.G., 1999, "Strength and ductility of concrete encased composite columns," Journal of Structural Engineering, Vol. 125, No. 9, pp. 1009-1019.

El-Tawil, S., Vidarsson, E., Mikesell, T., and Kunnath, S.K., 1999, "Inelastic behavior and design of steel panel zones," Journal of Structural Engineering, Vol. 125, No. 2, pp. 183-193.

El-Zanaty, M.H., Murray, D.W., and Bjorhovde, R., 1980, Inelastic Behavior of Multistory Steel Frames, Struct. Eng. Rep. No. 83, University of Alberta, Edmonton, Alberta, Canada.

Engelhardt, M.D., Fry, D.T., and Venti, M.J., 2000, Behavior and Design of Radius Cut Reduced Beam Section Connections, SAC Steel Project Document, Report No. SAC/BD-00/17, prepared by the SAC Joint Venture, a partnership of the Structural Engineers Association of California (SEAOC), Applied Technology Council (ATC), and California Universities for Research in Earthquake Engineering (CUREe).

Fahmy, M., 1999, Seismic Behavior of Moment-Resisting Steel Column Bases, Ph.D. Dissertation, Department of Civil and Environmental Engineering, University of Michigan, Ann Arbor, Michigan. 
Fahmy, M., Stojadinovic, B., and Goel, S.C., 1999, "Analytical and experimental studies on the seismic response of steel column bases," Proceedings, $8^{\text {th }}$ Canadian Conference on Earthquake Engineering, Vancouver, British Columbia.

Fahmy, M., Goel, S.C., and Stojadinovic, B., 2000, "Hysteretic behavior of steel moment resisting column bases," Proceedings, Third International Conference on Behavior of Steel Structures in Seismic Areas, STESSA 2000, Montreal, Canada.

FEMA, 2000a, Recommended Seismic Design Criteria for New Steel Moment Frame Buildings," FEMA 350 Report, prepared by the SAC Joint Venture, a partnership of the Structural Engineers Association of California (SEAOC), Applied Technology Council (ATC), and California Universities for Research in Earthquake Engineering (CUREe), for the Federal Emergency Management Agency, Washington, D.C.

FEMA, 2000b, State of the Art Report on Systems Performance of Moment Steel Frame Buildings in Earthquakes, FEMA 355C Report, prepared by the SAC Joint Venture, a partnership of the Structural Engineers Association of California (SEAOC), Applied Technology Council (ATC), and California Universities for Research in Earthquake Engineering (CUREe), for the Federal Emergency Management Agency, Washington, D.C.

FEMA, 2000c, State of the Art Report on Connection Performance, FEMA 355D Report, prepared by the SAC Joint Venture, a partnership of the Structural Engineers Association of California (SEAOC), Applied Technology Council (ATC), and California Universities for Research in Earthquake Engineering (CUREe), for the Federal Emergency Management Agency, Washington, D.C.

FEMA, 2000d, State of the Art Report on Past Performance of Steel Moment-Frame Buildings in Earthquakes, FEMA 355E Report, prepared by the SAC Joint Venture, a partnership of the Structural Engineers Association of California (SEAOC), Applied Technology Council (ATC), and California Universities for Research in Earthquake Engineering (CUREe), for the Federal Emergency Management Agency, Washington, D.C.

FEMA, 2000e, Prestandard and Commentary for the Seismic Rehabilitation of Buildings, FEMA 356 Report, prepared by the American Society of Civil Engineers for the Federal Emergency Management Agency, Washington, D.C.

FEMA, 2009a, Effects of Strength and Stiffness Degradation on Seismic Response, FEMA P-440A Report, prepared by the Applied Technology Council for the Federal Emergency Management Agency, Washington, D.C. 
FEMA, 2009b, Quantification of Building Seismic Performance Factors, FEMA P-695 Report, prepared by the Applied Technology Council for the Federal Emergency Management Agency, Washington, D.C.

FEMA, 2009c, NEHRP Recommended Seismic Provisions for New Buildings and Other Structures, FEMA P-750 Report, Federal Emergency Management Agency, Washington, D.C.

Fielding, D.J. and Huang, J.S., 1971, "Shear in steel beam-to-column connections," the Welding Journal, Vol. 50, No. 7 (research supplement).

Foliente, G., 1995, "Hysteresis modeling of wood joints and structural systems," Journal of Structural Engineering, Vol. 121, No. 6, pp. 1013-1022.

Foutch, D.A. and Shi, S., 1996, Connection Element (type 10) for DRAIN-2DX, Department of Civil Engineering, University of Illinois at UrbanaChampaign.

Fry, G.T., Jones, S.L., and Holliday, S.D., 1997, Protocol for Fabrication, Inspection, Testing, and Documentation of Beam-Column Connection Tests and Other Experimental Specimens, SAC Steel Project, Report No. SAC/BD-97/02, prepared by the SAC Joint Venture, a partnership of the Structural Engineers Association of California (SEAOC), Applied Technology Council (ATC), and California Universities for Research in Earthquake Engineering (CUREe).

Fukumoto, Y. and Galambos, T.V., 1966, "Inelastic lateral-torsional buckling of beam-columns," Journal of the Structural Division, Vol. 92, No. ST2, pp. 41-62.

Fukumoto, Y., Nethorcot, D.A., and Galambos, T.V., 1983, "Experimental data for the buckling of steel structures-NDSS stability of metal structures," Third International Colloquium proceedings: stability of metal structures, Structural Stability Research Council (SSRC), Rolla, Missouri, pp. 609-630.

Inai, E., Mukai, A., Kai, M., Tokinoya, H., Fukumoto, T., and Mori, K., 2004, "Behavior of concrete-filled steel tube beam columns," Journal of Structural Engineering, Vol. 130, No. 2, pp. 189-202.

Galambos, T.V., 1960, "Influence of partial base fixity on frame stability," Journal of the Structural Division, Vol. 86, No.ST5, pp. 85-108.

Galambos, T.V. and Ketter, R.L., 1961, "Columns under combined bending and thrust," Transactions of the American Society of Civil Engineering, Vol. 126, Part I, pp. 1-25. 
Galambos, T.V., Adams, P.F., and Fukumoto, Y., 1965, Further Studies on the Lateral Torsional Buckling of Steel Beam-Columns, Fritz Eng. Lab. Rep. No. 205A.36, Lehigh University, Bethlehem, Pennsylvania.

Gerard, G. and Becker, H., 1957, Handbook of Structural Stability, six parts, NACA Tech. Notes Nos. 3781-3786, National Advisory Committee for Aeronautics, Washington, D.C.

Gilton, C., Chi, B., and Uang, C.M., 2000, Cyclic Response of RBS Moment Connections: Weak-Axis Configuration and Deep Column Effects, Rep. No. SSRP-00/03, University of California, San Diego, California.

Gioncu, V. and Petcu, D., 1997, “Available rotation capacity of wide-flange beams and beam-columns: Part 1, theoretical approaches," Journal of Constructional Steel Research, Vol. 43, Nos.1-3, pp. 161-217.

Gomez, I.R., 2007, Behavior and Design of Column Base Connections, Ph.D. Dissertation, Department of Civil and Environmental Engineering, University of California, Davis, California.

Gourley, B.C., Hajjar, J.F., and Schiller, P.H., 1995, A Synopsis of Studies of the Monotonic and Cyclic Behavior of Concrete-Filled Steel Tube BeamColumns, Rep. No. ST-93-5, Department of Civil and Mineral Engineering, University of Minnesota, Minneapolis, Minnesota.

Grauvilardell, J.E., Lee, D., Hajjar, J.F., Dexter, R.J., 2005, Synthesis of Design, Testing and Analysis Research on Steel Column Base Plate Connections in High-Seismic Zones, Report No. ST-04-02., Department of Civil Engineering, University of Minnesota, Minneapolis, Minnesota.

Graves-Smith, T.R., 1969, "The ultimate strength of locally buckled columns of arbitrary length," in Thin-Walled Steel Structures, eds. K.C. Rockey and H.V. Hill, Crosby Lockwood, London.

Gross, J.L., Engelhardt, M.D., Uang, C.M., Kasai, K., and Iwankiw, N., 1999, Modification of Existing Welded Steel Moment Frame Connections for Seismic Resistance, Steel Design Guide Series 12, American Institute of Steel Construction, Chicago, Illinois.

Gupta, A. and Krawinkler, H., 1999, "Prediction of Seismic Demands for SMRFs with Ductile Connections and Elements," SAC Steel Project Background Document, Report No. SAC/BD-99/06, prepared by the SAC Joint Venture, a partnership of the Structural Engineers Association of California (SEAOC), Applied Technology Council (ATC), and California Universities for Research in Earthquake Engineering (CUREe). 
Gupta, A. and Krawinkler, H., 2000, "Behavior of ductile SMRFs at various seismic hazard levels," Journal of Structural Engineering, Vol. 126, No. 1, pp. 98107.

Hajjar, J.F. and Gourley, B.C., 1996, "Representation of concrete-filled steel tube cross-section strength," Journal of Structural Engineering, Vol. 122, No. 11, pp. 1327-1336.

Hajjar, J.F. and Gourley, B.C., 1997, "A cyclic nonlinear model for concrete-filled tubes. I. Formulation," Journal of Structural Engineering, Vol. 123, No. 6, pp. 736-744.

Hajjar, J.F., Gourley, B.C., and Olson, M.C., 1997, “A cyclic nonlinear model for concrete-filled tubes. II. Verification," Journal of Structural Engineering, Vol. 123, No. 6, pp. 745-754.

Hajjar, J.F., Leon, R.T., Gustafson, M.A., and Shield, C.K., 1998, "Seismic response of composite moment-resisting connections. II: Behavior," Journal of Structural Engineering, Vol. 124, No. 8, pp. 877-885.

Hall, D.H, 1981, "Proposed steel column strength criteria," Journal of Structural Division, Vol. 107, No. ST4, pp. 649-670.

Hancock, G.J., 1978, "Local, distortional, and lateral buckling of I-beams," Journal of the Structural Division, Vol. 104, No. 11, pp. 1787-1798.

Hancock, G.J., 1980, Design Methods for Thin-Walled Box Columns, Rep. No. 359, University of Sydney, Australia.

Hancock, G.J., 1981, "Interaction buckling of I-section columns," Journal of the Structural Division, Vol. 107, No. ST1, pp. 165-181.

Hancock, G.J., Kwon, Y.B., and Stefan Bernard, E., 1994, "Strength design curves for thin-walled sections undergoing distortional buckling," Journal of Constructional Steel Research, Vol. 31, Nos. 2-3, pp. 169-186.

Hawkins, N.M., 1968a, "The bearing strength of concrete loaded through rigid plates," Magazine of Concrete Research, Vol. 20, No. 62, pp. 31-40.

Hawkins, N.M., 1968b, "The bearing strength of concrete loaded through flexible plates," Magazine of Concrete Research, Vol. 20, No. 63, pp. 95-102.

Hellesland, J. and Bjorhovde, R., 1996a, "Restraint demand factors and effective length of braced columns," Journal of Structural Engineering, Vol. 122, No. 10, pp. 1216-1224.

Hellesland, J. and Bjorhovde, R., 1996b, "Improved frame stability analysis with effective lengths," Journal of Structural Engineering, Vol. 122, No. 11, pp. 1275-1283. 
Hensman, J. and Nethorcot, D.A., 2000, "Utilization of test data for column bases when assessing overall frame response: A review," Advances in Structural Engineering, Vol. 3, No. 1, pp. 7-17.

Hibbitt, Karisson and Sorensen (HKS), 2005, ABAQUS Version 6.5 for Advanced Mechanical Simulation, Pawtucket, Rhode Island.

Hitaka, T., Suita, K., and Kato, M., 2003, "CFT Column base design and practice in Japan," Proceedings of the International Workshop on Steel and Concrete Composite Construction (IWSCCC-2003), Report No. NCREE-03-026, National Center for Research in Earthquake Engineering, Taipei, Taiwan.

Ibarra, L.F. and Krawinkler, H., 2005, Global Collapse of Frame Structures Under Seismic Excitations, Report No. TB 152, the John A. Blume Earthquake Engineering Center, Stanford University, Stanford, California.

Ibarra, L., Medina, R., Krawinkler, H., 2002, "Collapse assessment of deteriorating SDOF systems," Proceedings of the $12^{\text {th }}$ European Conference on Earthquake Engineering, Paper No. 665, Elsevier Science Ltd., Oxford, United Kingdom.

Ibarra, L.F., Medina, R.A., and Krawinkler, H., 2005, "Hysteretic models that incorporate strength and stiffness deterioration," Journal of Earthquake Engineering and Structural Dynamics, Vol. 34, No. 12, pp. 1489-1511.

Ishizawa, T. and Iura, M., 2005, "Analysis of tubular steel bridge piers," Earthquake Engineering and Structural Dynamics, Vol. 34, No. 8, pp. 985-1004.

Iwan, W.D., 1966, "A distributed-element model for hysteresis and its steady-state dynamic response," Journal of Applied Mechanics, Vol. 33, No. 42, pp. 893900 .

Jaspart, J.P. and Vandegans, D., 1998, “Application of component method to column bases," Journal of Constructional Steel Research, Vol. 48, pp. 89-106.

Jin, J. and El-Tawil, S., 2003, "Inelastic cyclic model for steel braces," Journal of Engineering Mechanics, Vol. 129, No. 5, pp. 548-557.

Johnston, B.G. and Cheney, L., 1942, Steel Columns of Rolled Wide Flange Sections, Prog. Rep. No. 2, American Institute of Steel Construction, Chicago, Illinois.

Johnston, B.G., 1976, Guide to Stability Design Criteria for Metal Structures, $3^{\text {rd }}$ Edition, Wiley, New York.

Jones, S.W., Kirby, P.A., and Nethercot, D.A., 1980, "Effect of semi-rigid connections on steel column strength," Journal of Constructional Steel Research, Vol. 1, No. 1, pp. 35-46. 
Jones, S.W., Kirby, P.A., and Nethercot, D.A., 1982, "Columns with semi-rigid joints," Journal of the Structural Division, Vol. 108, No. ST2, pp. 361-372.

Kawaguchi, J. and Morino, S., 2001, "Experimental study on post - local buckling behavior of beams-columns under cyclic loading," Journal of Structural and Construction Engineering, No. 540, pp. 141-148.

Kawashima, K., Hasegawa, K., MacRae, G.A., and Ikeuchi, T., 1992, "Ductility of steel bridge piers from dynamic loading tests," in Stability of Steel Structures Under Cyclic Loading, eds. T. Fukumoto and G.C. Lee, CRC Press, Boca Raton, Florida.

Kemp, A.R., 1984, "Slenderness limits normal to the plane-of-bending for beamcolumns in plastic design," Journal of Constructional Steel Research, Vol. 4, pp. $135-150$.

Ketter, R.L., Beedle, L.S., and Johnson, B.G., 1952, "Welded continuous frames and their components," the Welding Journal Research Supplement, Vol. 31, No. 12, pp. 607-622.

Ketter, R.L., Kaminsky, E.L., and Beedle, L.S., 1955, "plastic deformation of wideflange beam columns," Transactions of the American Society of Civil Engineering, Vol. 120, p. 1028.

Ketter, R.L., 1962, "Further studies of the strength of beam-columns," Transactions of the American Society of Civil Engineering, Vol. 127, Part II, pp. 224-466.

Kim, K.D. and Engelhardt, M.D., 2002, "Monotonic and cyclic loading models for panel zones in steel moment frames," Journal of Constructional Steel Research, Vol. 58, pp. 605-635.

Kishi, N. and Chen, W.F., 1986, Data Base of Steel Beam-to-Column Connections, Struct. Eng. Rep. No. CE-STR-86-26, School of Civil Engineering, Purdue University, West Lafayette, Indiana.

Kishi, N. and Chen, W.F., 1990, "Moment-rotation relations of semi-rigid connections with angles," Journal of Structural Engineering, Vol. 116, No. 7, pp. 1813-1834.

Kishi, N., Chen, W.F., Goto, Y., and Matsuoka, K.G., 1993, "Design aid of semirigid connections for frame analysis," Engineering Journal, Vol. 30, No. 3, pp. 90-103.

Krawinkler, H., 1978, "Shear in beam-column joints in seismic design of steel frames," Engineering Journal, Vol. 15, No. 3, pp. 82-91.

Krawinkler, H., 2000, "System performance of steel moment resisting frame structures," Proceedings, $12^{\text {th }}$ World Conference on Earthquake Engineering, Auckland, New Zealand. 
Krawinkler, H., 2010, Loading Histories for Cyclic Tests in Support of Performance Assessment of Structural Components, PEER Annual Meeting, San Francisco, California.

Krawinkler, H., Bertero, V.V., and Popov, E.P., 1971, Inelastic Behavior of Steel Beam-to- Column Subassemblages, Report EERC-71-7, University of California, Berkeley, California.

Krawinkler, H. and Mohasseb, S., 1987, "Effects of panel zone deformations on seismic response," Journal of Constructional Steel Research, Vol. 8, pp. 233250 .

Krawinkler, H., Parisi, F., Ibarra, L., Ayoub, A., Medina, R., 2000b, "Development of a testing protocol for woodframe structures," Rep. No. W-O2, Consortium of Universities for Research in Earthquake Engineering (CUEE), Richmond, California.

Krishnan, S., 2003, FRAME3D - A Program for Three-Dimensional Nonlinear TimeHistory Analysis of Steel Buildings: User Guide, Rep. EERL 2003-03, Earthquake Engineering Research Laboratory, California Institute of Technology, Pasadena, California.

Krishnan, S., 2010, "Modified elastofiber element for steel slender column and brace modeling," Journal of Structural Engineering, Vol. 136, No. 11, pp. 13501366.

Krishnan, S. and Hall, J.F., 2006, "Modeling steel frame buildings in three dimensions. I: Panel zone and plastic hinge beam elements," Journal of Structural Engineering, Vol. 132, Vol. 4, pp. 345-358.

Kroll, W.D., Fisher, G.P., and Heimerl, G.J., 1943, Charts for the Calculation of the Critical Stress for Local Instability of Columns with I, Z, Channel and Rectangular Tube Sections, NACA Wartime Rep. No. L-429, National Advisory Committee for Aeronautics, Washington, D.C.

Kunnath, S.K., Reinhorn, A.M., and Park, Y.J., 1990, “Analytical modeling of inelastic seismic response of R/C structures," Journal of Structural Engineering, Vol. 116, No. 4, pp. 996-1017.

Kunnath. K., Reinhorn, A.M., and Lobo, R.F., 1992, “IDARC Version 3.0: A Program Inelastic Damage Analysis Of Reinforced Concrete Structures," Report No. NCEER-92-0022, University at Buffalo, State University of New York.

Kurata, M., Nakashima, M., and Suita, K., 2005, "Effect of column base behavior on the seismic response of steel moment frames," Journal of Earthquake Engineering, Vol. 9, Special Issue 2, pp. 415-438. 
Kwasniewski, L, Stojadinovic, B., and Goel, S.C., 1999, Local and Lateral-Torsion Buckling of Wide Flange Beams, Report SAC/BD-99/20, prepared by the SAC Joint Venture, a partnership of the Structural Engineers Association of California (SEAOC), Applied Technology Council (ATC), and California Universities for Research in Earthquake Engineering (CUREe).

Lamarche, C.F. and Tremblay, R., 2011, "Seismically induced cyclic buckling of steel columns including residual-stress and strain-rate effects," Journal of Constructional Steel Research, Vol. 67, No. 9, pp. 1401-1410.

Lay, M.G., 1974, Source Book for the Australian Steel Structures Code AS1250, Australian Institute of Steel Construction, Sydney, Australia.

Lay, M.G. and Galambos, T.V., 1965, "The experimental behaviour of retrained columns," Welding Research Council Bulletin, No. 110.

Lau, S.C.W. and Hancock, G.J., 1987, "Distortional buckling formulas for channel columns," Journal of Structural Engineering, Vol. 113, No. 5, pp. 10631078.

Lee, C.H., Jeon, S.W., Kim, J.H., Uang, C.M., 2005, "Effects of panel zone strength and beam web connection method on seismic performance of reduced beam section steel moment connections," Journal of Structural Engineering, Vol. 131, No. 12, pp. 1854-1865.

Lee, D., Cotton, S.C., Hajjar, J.F., Dexter, R.J., 2005a, “Cyclic behavior of steel moment-resisting connections reinforced by alternative column stiffener details I. Connection performance and continuity plate detailing," Engineering Journal, pp. 189-214.

Lee, D., Cotton, S.C., Hajjar, J.F., Dexter, R.J., 2005b, "Cyclic behavior of steel moment-resisting connections reinforced by alternative column stiffener details II. Panel zone behavior and doubler plate detailing," Engineering Journal, pp. 215-238.

Lee, K. and Foutch, D.A., 2000, Performance Prediction and Evaluation of Special Moment Frame Buildings for Seismic Loads, SAC Steel Project Background Rep. No. SAC/BD-00/25, prepared by the SAC Joint Venture, a partnership of the Structural Engineers Association of California (SEAOC), Applied Technology Council (ATC), and California Universities for Research in Earthquake Engineering (CUREe).

Lee, K. and Foutch, D.A., 2002a, "Performance evaluation of new steel frame buildings for seismic loads," Earthquake Engineering and Structural Dynamics, Vol. 31, pp, 653-670. 
Lee, K. and Foutch, D.A., 2002b, "Seismic performance evaluation of pre-Northridge steel frame buildings with brittle connections," Journal of Structural Engineering, Vol. 128, No. 4, pp. 546-555.

Lee, D. and Goel, S.C., 2001, Seismic Behavior of Column-Base Plate Connections Bending about Weak Axis, Report No. UMCEE 01-09, Department of Civil and Environmental Engineering, University of Michigan, Ann Arbor, Michigan.

Lee, K. and Stojadinovic, B., 2004, "Low-cycle fatigue limit on seismic rotation capacity for US Steel moment connections," Proceedings, $13^{\text {th }}$ World Conference on Earthquake Engineering, Vancouver, British Columbia.

Lee, S.J., 1987, Seismic Behavior of Steel Building Structures with Composite Slabs, Ph.D. Dissertation, Department of Civil Engineering, Lehigh University, Bethlehem, Pennsylvania.

Leon, R. and White, D.W, 1998, "Three-dimensional Slab Effects in Partially Restrained Composite Construction," Presentation at $5^{\text {th }}$ JTCC Meeting, Tokyo, Japan.

Leon, R.T., Hajjar, J., Gustafson, M.A., and Shield, C.M., 1998, "Seismic response of composite moment-resisting connections, I: Performance, II: Behavior," Journal of Structural Engineering, Vol. 124, No. 8.

Liew, J.Y.R., White, D.W., and Chen, W.F., 1993, "Second-order refined plastic hinge analysis for frame design, Parts 1 and 2," Journal of Structural Engineering, Vol. 119, No. 11, pp. 3196-3237.

Lignos, D.G. and Krawinkler, H., 2007, “A database in support of modeling of component deterioration for collapse prediction of steel frame structures," Proceedings, ASCE Structures Congress, Long Beach, California.

Lignos, D.G. and Krawinkler, H., 2009, Sidesway Collapse of Deteriorating Structural Systems Under Seismic Excitations, Report No. 172, John A. Blume Earthquake Engineering Research Center, Stanford University, Stanford, California.

Lignos, D.G. and Krawinkler, H., 2011, "Deterioration modeling of steel components in support of collapse prediction of steel moment frames under earthquake loading," Journal of Structural Engineering, Vol. 137, No. 11, pp. 12911302.

Lignos, D.G., Kolios, D., and Miranda, E., 2010a, "Fragility assessment of reduced beam section moment connections," Journal of Structural Engineering, Vol. 136, No. 9, pp. 1140-1150. 
Lignos, D.G., Krawinkler, H. and Whittaker, A., 2011a, "Prediction and validation of sidesway collapse of two scale models of a 4-story steel moment frame," Earthquake Engineering and Structural Dynamics, Vol. 40, No. 7, pp. 807825.

Lignos, D.G., Putman, C., Zareian, F., and Krawinkler, H., 2011b, "Seismic evaluation of steel moment frames and shear walls using nonlinear static analysis procedures," Proceedings, ASCE Structures Congress, Las Vegas, Nevada.

Lignos, D.G., Chung, Y.L., Nagae, T., Nakashima, M., 2011c, "Numerical and experimental evaluation of seismic capacity of high-rise steel buildings subjected to long duration earthquakes," Computers and Structures, Vol. 89, Nos. 11-12, pp. 959-967.

Lignos, D.G., Hikino, T., Matsuoka, Y., and Nakashima, M., 2011d, "Collapse assessment of steel moment frames based on e-defense full scale shake table collapse tests," Journal of Structural Engineering (under review).

Lignos, D.G., Eads, L., and Krawinkler, H., 2011e, "Effect of composite action on collapse capacity of steel moment frames under cyclic loading," Proceedings, Eurosteel, Budapest, Hungary.

Lignos, D.G., Luna-Moreno, M.D., and Billington, S.L., 2011f, "Seismic retrofit of existing steel moment resisting frames with innovative materials: large scale hybrid simulation tests," Proceedings, $3^{\text {rd }}$ International Conference on Computational Methods in Structural Dynamics and Earthquake Engineering, COMPDYN 11, Corfu, Greece.

Lindner, J., 1972, "Theoretical investigations of columns under biaxial loading," Proceedings, Int. Colloq. Column Strength, IABSE-CRC-ECCS, Paris, France.

Little, G.H., 1979, “The strength of square steel box-columns: Design curves and their theoretical basis," Structural Engineer, Vol. 57, No. 2, pp. 49-61.

Liu, J. and Astaneh, A., 2000, "Cyclic testing of simple connections including effects of slab," Journal of Structural Engineering, Vol. 126, No.1, pp. 32-39.

Lobo, R. F., 1994, Inelastic Dynamic Analysis of Reinforced Concrete Structures in Three Dimensions, Ph.D. Dissertation, Dept. of Civil Engineering, University at Buffalo, State University of New York.

Lu, L.W. and Kamalvand, H., 1968, "Ultimate strength of laterally loaded columns," Journal of the Structural Division, Vol. 94, No. ST6, pp. 1505-1524. 
Lu, L.W., Shen, Z.Y., and Hu, X.R., 1983, Inelastic Instability Research at Lehigh University: Instability and Plastic Collapse of Steel Structures, ed. L.J. Morris, Granada, London, United Kingdom.

Luco, N. and Cornell, C.A., 2000, "Effects of connection fractures on SMRF seismic drift demands," Journal of Structural Engineering, Vol. 126, No. 1, pp. 127136.

MacRae, G.A, 1999, Parametric Study of the Effect of Seismic Demands of Ground Motion Intensity and Dynamic Characteristics," report to the SAC Joint Venture, a partnership of the Structural Engineers Association of California (SEAOC), Applied Technology Council (ATC), and California Universities for Research in Earthquake Engineering (CUREe), for the Federal Emergency Management Agency, Washington, D.C.

MacRae, G.A. and Mattheis, J., 2000, “Three-dimensional steel building response to near-fault motions," Journal of Structural Engineering, Vol. 126, No. 1, pp. $117-126$.

MacRae, G.A., Carr, A.J., and Walpole, W.R., 1990, The Seismic Response of Steel Frames, Report No. 90-6, Department of Civil Engineering Research, University of Canterbury, Christchurch, New Zealand.

MacRae, G.A., Hyde, K., Walpole, W, Moss, P. Hyland, C., Clifton, G.C., Mago, N., 2006, "Column axial shortening effects in steel frames," Proceedings, New Zealand Society for Earthquake Engineering (NZSEE) Conference, Napier, New Zealand.

Mamaghani, I.H.P., 2008, "Seismic design and ductility evaluation of thin-walled steel bridge piers of box sections," Transportation Res. Record: Journal of Transportation Res. Board, No. 2050, pp. 137-142.

Marson, J. and Bruneau, M., 2004, "Cyclic testing of concrete-filled circular steel bridge piers having encased fixed-based detail," Journal of Bridge Engineering, Vol. 9, No. 1, pp. 14-23.

Mason, R.G., Fisher, G.P., and Winter, G., 1958, "Eccentrically loaded, hinged steel columns," Journal of the Engineering Mechanics Division, Vol. 84, No. EM4, p. 1792.

Mazzolani, F.M. and Gioncu, V., 2002, Ductility of Seismic Resistant Steel Structures, Technology and Engineering, Spon Press, New York, New York.

McFalls, R.K. and Tall, L., 1970, “A study of welded columns manufactured from flame-cut plates," Welding Journal, AWS, Vol. 49, No. 4, pp. 141s-153s.

McKenna, F., 1997, Object Oriented Finite Element Programming Frameworks for Analysis, Algorithms and Parallel Processing, Ph.D. Dissertation, 
Department of Civil and Environmental Engineering, University of California, Berkeley, California.

Medina, R. and Krawinkler, H., 2003, Seismic Demands for Non-Deteriorating Frame Structures and Their Dependence on Ground Motions, Report No. TR 144, John A. Blume Earthquake Engineering Center, Stanford University, Stanford, California.

Miranda, C. and Ojalvo, M., 1965, "Inelastic lateral-torsional buckling of beamcolumns," Journal of the Engineering Mechanics Division, Vol. 91, No. EM6, pp. 21-38.

Morino, S., Kawaguchi, J., Tsuji, A., and Kadoya, H., 2003, "Strength and stiffness of CFT semi-embedded type column base," Proceedings, ASSCCA 2003 Conference, Sydney, Australia.

Möller, M., Johansson, B., and Collin, P., 1997, “A new analytical model of inelastic local flange buckling," Journal of Constructional Steel Research, Vol. 23, Nos. 1-3, pp. 43-63.

Mostaghel, N., 1999, "Analytical description of pinching, degrading hysteretic systems,” Journal of Engineering Mechanics, Vol. 125, No. 2, pp. 216-224.

Myers, A.T., Kanvinde, A.M., Fell, B.V., and Fu, X., 2007, "Large scale tests and micromechanics-based models to characterize ultra low cycle fatigue in welded structural details," Proceedings, ASCE Structures Congress, Long Beach, California.

Myers, A.T., Kanvinde, A.M., Deierlein, G.G., and Fell, B.V., 2009, "Effect of weld details on the ductility of steel column base plate connections," Journal of Constructional Steel Research, Vol. 65, pp. 1366-1373.

Murray, T.M., 1983, "Design of lightly loaded column base plates," Engineering Journal, Vol. 20, No. 4, pp. 143-152.

Naka, T., Kato, B., Watabe, M., and Nakao, M., 1969, "Research on the behavior of steel beam-to-column connections in the seismic-resistant structure," Proceedings, Fourth World Conference on Earthquake Engineering, Santiago, Chile.

Nakashima, M., 1994, "Variation of ductility capacity of steel beam-columns," Journal of Structural Engineering, Vol. 120, No. 7, pp. 1941-1960.

Nakashima, S., 1996, "Response of steel column bases embedded shallowly into foundation beams," Proceedings, Eleventh World Conference on Earthquake Engineering, Acapulco, Mexico.

Nakashima, S. and Igarashi, S., 1986, "Behavior of steel square tubular column bases embedded in concrete footings under bending moment and shearing force: 
Part 1 - Test program and load-displacement relations," Journal of Structural and Construction Engineering, No. 366, pp. 106-118.

Nakashima, S. and Igarashi, S., 1987, "Behavior of steel square tubular column bases of corner columns embedded in concrete footings," Proceedings, International Conference on Steel and Aluminum Structures, Cardiff, United Kingdom.

Nakashima, M. and Liu, D., 2005, "Instability and complete failure of steel columns subjected to cyclic loading," Journal of Engineering Mechanics, Vol. 131, No. 6.

Nakashima, M., Kanao, I., and Liu, D., 2002, "Lateral instability and lateral bracing of steel beams subjected to cyclic loading," Journal of Structural Engineering, Vol. 128, No. 10, pp. 1308-1316.

Nakashima, M., Matsumiya, T., Suita, K., and Liu, D., 2006, “Test on full-scale three-story steel moment frame and assessment of ability of numerical simulation to trace cyclic inelastic behavior," Earthquake Engineering and Structural Dynamics, Vol. 35, No. 1, pp. 3-19.

Nakashima, M. and Sawaizumi, S., 1999, "Effect of column-to-beam strength ratio on earthquake responses of steel moment frames, Parts 1 and 2," Journal of Steel Construction Engineering, Vol. 6, No. 4 (in Japanese).

Nakashima, M. and Sawaizumi, S., 2000, "Column-to-beam strength ratio required for ensuring beam-collapse mechanisms in earthquake responses of steel moment frames" Proceedings, $12^{\text {th }}$ World Conference on Earthquake Engineering, Auckland, New Zealand.

Nakashima, M., Takanashi, K., and Kato, H., 1990, "Tests of steel beam-columns subject to sidesway," Journal of Structural Engineering, Vol. 116, No. 9, pp. 2516-2531.

Neuenhofer, A. and Fillipou, F., 1997, "Evaluation of nonlinear frame finite element models," Journal of the Structural Division, Vol. 123, No. 7, pp. 958-966.

NZSEE, 2006, Assessment and Improvement of the Structural Performance of Buildings in Earthquakes, Recommendations of a NZSEE Study Group on Earthquake Risk Buildings, New Zealand Society of Earthquake Engineering, Wellington, New Zealand.

Newell, J. and Uang, C.M., 2006, Cyclic Behavior of Steel Columns with Combined High Axial Load and Drift Demand, Report No. SSRP-06/22, Department of Structural Engineering, University of California, San Diego, California. 
Newell, J. and Uang, C.M., 2008, "Cyclic behavior of steel wide-flange columns subjected to large drift," Journal of Structural Engineering, Vol. 134, No. 8, pp. 1334-1342.

NIST, 1999, Research Required to Support Full Implementation of PerformanceBased Seismic Design, NIST GCR 09-917-2, prepared by the Building Seismic Safety Council of the National Institute of Building Sciences for the National Institute of Standards and Technology, Gaithersburg, Maryland.

NIST, 2010, Evaluation of the FEMA P-695 Methodology for Quantification of Building Seismic Performance Factors, NIST GCR 10-917-8, prepared by the NEHRP Consultants Joint Venture, a partnership of the Applied Technology Council and the Consortium of Universities for Research in Earthquake Engineering for the National Institute of Standards and Technology, Gaithersburg, Maryland.

NIST, 2011, Nonlinear Structural Analysis for Seismic Design: A Guide for Practicing Engineers, NEHRP Seismic Design Technical Brief No. 4, prepared by the NEHRP Consultants Joint Venture, a partnership of the Applied Technology Council and the Consortium of Universities for Research in Earthquake Engineering for the National Institute of Standards and Technology, Gaithersburg, Maryland.

Nukala, P.K.V.V. and White, D.W., 2004, "A mixed finite element for threedimensional nonlinear analysis of steel frames," Computer Methods Applied Mechanics and Engineering, Vol. 193, Nos. 23-26, pp. 2507-2545.

NZS, 2004, Structural Design Actions, Part 5: Earthquake Actions, NZ 1170.5:2004, New Zealand Standards, Wellington, New Zealand.

NZS, 2007, Steel Structures Standard, Incorporating Amendment No. 1 and Amendment No. 2, NZ 3404: Parts 1 and 2:1997, New Zealand Standards, Wellington, New Zealand.

Papangelis, J.P. and Hancock, G.J., 1995, "Computer analysis of thin-walled structural members," Computers and Structures, Vol. 56, No. 1, pp. 157-176.

PEER/ATC, 2010, Modeling and Acceptance Criteria for Seismic Design and Analysis of Tall Buildings, PEER/ATC-72-1 Report, prepared by the Applied Technology Council in cooperation with the Pacific Earthquake Engineering Research Center, Redwood City, California.

Peng, B.H.H., MacRae, G.A., Walpole, W.R., Moss, P.J., and Dhakal, R., 2007, "Location of plastic hinges in columns of steel moment frames," Proceedings, New Zealand Society for Earthquake Engineering (NZSEE) Conference, Palmerston North, New Zealand. 
Peng, B.H.H., MacRae, G.A., Walpole, W.R., Moss, P.J., Dhakal, R., and Clifton, C., 2008, "Plastic hinge location in columns of steel moment frames subjected to seismic actions" Bulletin of the New Zealand Society for Earthquake Engineering, Vol. 41, No. 1.

Pertold, J., Xiao, R. Y., and Wald, F., 2000a, "Embedded steel column bases - i. experiments and numerical simulation," Journal of Constructional Steel Research, Vol. 56, pp. 253-270.

Pertold, J., Xiao, R.Y., and Wald, F., 2000b, "Embedded steel column bases - II. Design model proposal," Journal of Constructional Steel Research, Vol. 56, pp. 271-286.

Pi, Y.-L. and Trahair, N.S., 1992a, "Prebuckling deformations and lateral buckling: I. Theory," Journal of Structural Engineering, Vol. 118, No. 11, pp. 29492966.

Pi, Y.-L. and Trahair, N.S., 1992b, "Prebuckling deformations and lateral buckling: II. Applications," Journal of Structural Engineering, Vol. 118, No. 11, pp. 2967-2985.

Pi, Y.-L. and Trahair, N.S., 1994a, "Non-linear inelastic analysis of steel beamcolumns: I. Theory," Journal of Structural Engineering, Vol. 120, No. 7, pp. 2041-2061.

Pi, Y.-L. and Trahair, N.S., 1994b, "Non-linear inelastic analysis of steel beamcolumns: II. Application," Journal of Structural Engineering, Vol. 120, No. 7, pp. 2062-2085.

Picard, A. and Beaulieu, D., 1985, "Behavior of a simple column base connection," Canadian Journal of Civil Engineering, Vol. 12, pp. 126-136.

Pillai, U.S., 1974, "Beam columns of hollow sections," Journal of Canadian Society for Civil Engineering, Vol. 1, No. 2, pp. 194-198.

Popov, E.P. and Pinkney, R.B., 1969, "Cyclic yield reversals in steel building connections," Journal of Structural Engineering, Vol. 95, No. ST3, pp 327353.

Popov, E.P. and Stephen, R.M., 1970, Cyclic Loading of Full Size Steel Connections, Report EERC-70-3, University of California, Berkeley, California.

Popov, E.P. and Stephen, R.M., 1977a, "Capacity of columns with splice imperfections," Engineering Journal, Vol. 14, No. 1, pp. 16-23.

Popov, E.P. and Stephen, R.M., 1977b, “Tensile capacity of partial penetration welds," Journal of Structural Division, Vol. 103, No. ST9, pp. 1721-1729. 
Popov, E.P., Amin, N.R., Louie, J.C., and Stephen, R.M., 1985, "Cyclic behavior of large beam-column assemblies," Earthquake Spectra, Vol. 1, No. 2, pp. 201237.

Popov, E.P., Bertero, V.V., and Chandramouli, S., 1975, Hysteretic Behavior of Steel Columns, Report EERC-75-11, University of California, Berkeley, California.

Rahnama, M. and Krawinkler, H., 1993, Effect of Soft Soils and Hysteresis Models on Seismic Design Spectra, Report No. 108, John A. Blume Earthquake Engineering Center, Stanford University, Stanford, California.

Rajasekaren, S., 1977, "Finite element method for plastic beam-columns," in Theory of Beam-Columns, eds. W.F. Chen and T. Atsuta, McGraw-Hill, New York, Chap. 12.

Ramberg, W. and Osgood, W.R., 1943, Description of Stress-Strain Curves by Three Parameters, Monograph No. 4, Publicazione Italsider, Nuova Italsider, Genova, Italy.

Razzaq, Z. and Chang, J.G., 1981, "Partially restrained imperfect columns," Proceedings, Conference on Joints Structural Steelwork, Teesside, England.

Reinhorn, A.M., Madan, A., Valles, R.E., Reichmann, Y., and Mander, J.B., 1995, Modeling of Masonry Infill Panels for Structural Analysis, Report No. NCEER-95-0018, University at Buffalo, State University of New York.

Reinhorn, A. M., Roh, H., Sivaselvan, M., Kunnath, S. K., Valles, R. E., Madan, A., Li, C., Lobo, R., and Park, Y. J., 2009, IDARC 2 D Version 7.0: A Program for the Inelastic Damage Analysis of Structures, Report No. MCEER-090006, University at Buffalo, State University of New York.

Rhodes, J. and Harvey, J.M., 1971, "Effects of eccentricity of load or compression on the buckling and post-buckling behavior of flat plates," International Journal of Mechanical Sciences, Vol. 13, pp. 867-879.

Ricles, J.M. and Paboojian, S.D., 1994, "Seismic performance of steel-encased composite columns," Journal of Structural Engineering, Vol. 120, No. 8, pp. 2474-2494.

Ricles, J.M., Mao, C., Lu, L.W., and Fisher, J.W., 2000, Development and Evaluation of Improved Ductile Welded Unreinforced Flange Connections, Report No. SAC/BD-00/24, prepared by the SAC Joint Venture, a partnership of the Structural Engineers Association of California (SEAOC), Applied Technology Council (ATC), and California Universities for Research in Earthquake Engineering (CUREe). 
Ricles, J.M., 2002, "Inelastic cyclic testing of welded unreinforced moment connections," Journal of Structural Engineering, Vol. 128, No. 4.

Ricles, J.M., Zhang, X., Lu, L.W., and Fisher, J., 2004a, Development of Seismic Guidelines for Deep-Column Steel Moment Connections, Report No. 04-13, Advanced Technology for Large Structural Systems Engineering Research Center, Lehigh University, Bethlehem, Pennsylvania.

Ricles, J.M., Peng, S.W., and Lu, L.W., 2004b, "Seismic behavior of composite concrete filled steel tube column-wide flange beam moment connections," Journal of Structural Engineering, Vol. 130, No. 2, pp. 223-232.

Robertson, A., 1925, "The strength of struts," Selected Engineering Papers, No. 28, The Institute of Civil Engineers, London, United Kingdom.

Roeder, C.W. and Assadi, M., 1982, "Lateral stability of I beams with partial support," Journal of Structural Division, Vol. 108, No.ST8, pp. 1768-1780.

Sato, K., 1987, A Research on the Aseismic Behavior of Steel Column Base for Evaluating Its Strength Capacity and Fixity, Report No. 69, Kajima Institute of Construction Technology, Tokyo, Japan.

Schafer, B.W., 2002, "Local, distortional, and euler buckling of thin-walled columns," Journal of Structural Engineering, Vol. 128, No. 3, pp. 289-299.

Schafer, B.W. and Pekoz, T., 1999, "Laterally braced cold-formed steel flexural members with edge stiffened flanges," Journal of Structural Engineering, Vol. 125, No. 2, pp. 118-126.

Schafer, B.W. and Adany, S., 2006, "Buckling analysis of cold-formed steel members using cufsm: conventional and constrained finite strip methods," Proceedings, Eighteenth International Specialty Conference on Cold-Formed Steel Structures, Orlando, Florida.

Shanley, F.R., 1947, "Inelastic column theory," Journal of Aeronautical Science, Vol. 14, No. 5, pp. 261.

Schellenberg, A.H., 2008, Advanced Implementation of Hybrid Simulation, Ph.D. Dissertation, Department of Civil and Environmental Engineering, University of California, Berkeley, California.

Schneider, S.P., Roeder, C.W., and Carpenter, J.E., 1993, "Seismic behavior of moment- resisting steel frames, analytical study and experimental investigation," Journal of Structural Engineering, Vol. 119, No. 6.

Shen, Z.Y. and Lu, L.W., 1983, “Analysis of initially crooked, end restrained columns," Journal of Constructional Steel Research, Vol. 3, No. 1, pp. 1018. 
Shen, J.H., Astaneh, A., and McCallen, D.B., 2002, Use of Deep Columns in Special Steel Moment Frames, Steel Tip Report No. 24, Structural Steel Educational Council, Moraga, California.

Silvestre, N. and Camotim, D., 2002, "Second-order generalised beam theory for arbitrary orthotropic materials," Thin-Walled Structures, Vol. 40, No. 9, pp. 791-820.

Sivaselvan, M. and Reinhorn, A.M., 2000, "Hysteretic models for deteriorating inelastic structures," Journal of Engineering Mechanics, Vol. 126, No. 6, pp. 633-640.

Skaloud, M. and Zornerova, M., 1970, "Experimental investigation into the interaction of the buckling of compressed thin-walled columns with the buckling of their plate elements," Acta Tecnica CSAV, No. 4.

Slutter, R.G., 1981, Tests of Panel Zone Behavior in Beam-Column Connections, Report No. 403.1, Fritz Engineering Lab., Lehigh University, Bethlehem, Pennsylvania.

Snijder, H.H. and Hoenderkamp, J.C.D., 2006, "Experimental tests on spliced columns for splice strength and stiffness requirements," Proceedings, Stability and Ductility of Steel Structures, Lisbon, Portugal.

Snijder, H.H. and Hoenderkamp, J.C.D., 2008, "The influence of end plate splices on the load carrying capacity of columns," Journal of Constructional Steel Research, Vol. 66, pp. 1261-1277.

Song, J. and Pincheira, J., 2000, "Spectral displacement demands of stiffness and strength degrading systems," Earthquake Spectra, Vol. 16, No. 4, pp. 817851 .

Spacone, E., Filippou, F.C., and Taucer, F.F., 1996, "Fiber beam-column model for nonlinear analysis of R/C frames. 1: Formulation," Earthquake Engineering and Structural Dynamics, Vol. 25, No. 7, pp. 711-725.

Stamatopoulos, G. and Ermopoulos, J., 1997, "Interaction curves for column baseplate connections," Journal of Constructional Steel Research, Vol. 44, Nos. 1-2, pp. 69-89.

Stowell, E.Z., 1948, A Unified Theory of Plastic Buckling of Columns and Plates, NACA Tech. Note No. 1556, National Advisory Committee for Aeronautics, Washington, D.C.

Stowell, E.Z., Heimerl, G.J., Libove, C., and Lundquist, E.E., 1952, “Buckling stresses for flat plates and sections," Transactions of the American Society of Civil Engineering, Vol. 117, pp. 545-578. 
Sugiyama, S. and Igarashi, K., 1986, "Experimental investigation of inelastic behavior of cold - formed rectangular hollow structural sections under bending and constant axial force," Journal of Structural and Construction Engineering.

Suita, K., Yamada, S., Tada, M., Kasai, K., Matsuoka, Y., and Sato, E., 2008, "Results of recent e-defense tests on full-scale steel buildings: Part 1 Collapse experiment on 4-story moment frame," Proceedings, ASCE Structures Congress, Vancouver, Canada.

Susantha, K.A.S., Ge, H., and Usami, T., 2002, "Cyclic analysis and capacity prediction of concrete-filled steel box columns," Earthquake Engineering and Structural Dynamics, Vol. 31, No. 2, pp. 195-216.

Surovek, A.E., White, D.W., and Leon, R.T., 2005, "Direct analysis for design evaluation of partially restrained steel framing systems," Journal of Structural Engineering, Vol. 131, No. 9, pp. 1376-1389.

Tall, L., 1964, "Recent developments in the study of column behavior," Journal of the Institute of Engineering, Vol. 36, No. 12, pp. 319-333.

Thambiratnam, D.P. and Paramasivam, P., 1986, "Base plates under axial loads and moments," Journal of Structural Engineering, Vol. 112, No. 5, pp. 11661181.

Thomasson, P.O., 1978, Thin-Walled C-Shaped Panels in Axial Compression, Document D.I. 1978, Swedish Council for Building Research, Stockholm.

Timoshenko, S.P. and Gere, J.M., 1961, Theory of Elastic Stability, $2^{\text {nd }}$ Edition, McGraw-Hill, New York.

Trahair, N.S., 1993, Flexural-Torsional Buckling of Structures, Taylor and Francis, London, United Kingdom.

Tort, C. and Hajjar, J.F., 2007, Reliability-Based Performance-Based Design of Rectangular Concrete-Filled Steel Tube (RCFT) Members and Frames, Structural Engineering Report No. ST-07-1, Department of Civil Engineering, University of Minnesota, Minneapolis, Minnesota.

Tremblay, R., Tchebotarev, N., and Filiatrault, A., 1997, "Seismic performance of RBS connections for steel moment resisting frames: influence of loading rate and floor slab," Proceedings, Second International Conference on the Behavior of Steel Structures in Seismic Areas, STESSA '97, Kyoto, Japan.

Tsuda, K. and Matsui, C., 1998, "Strength of square steel tubular beam-columns under constant vertical and horizontal loads," Journal of Structural and Construction Engineering, No. 512, pp. 149-156. 
Tsuji, B. and Nakatsura, T., 1986, "Safety criteria in design of tubular structures," Proceedings, International Meeting, July 11-12, 1986, Tokyo, Japan.

Uang, C.M. and Fan, C.C., 1999, Cyclic Instability of Steel Moment Connections with Reduced Beam Sections, Structural Steel Research Project Report No. SSRP99/21, University of California, San Diego, California.

Uang, C.M., Gilton, C., and Chi, B., 2000, Cyclic Response of RBS Moment Connections: Weak-Axis Configuration and Deep Column Effects, Structural Steel Research Project Report No. SSRP-00-03, University of California, San Diego, California.

Usami, T., Gao, S., and Ge, H., 2000, Stiffened steel box columns. Part 2: ductility evaluation," Earthquake Engineering and Structural Dynamics, Vol. 29, No. 11, pp. 1707-1722.

van Kuren, R.C. and Galambos, T.V., 1964, "Beam-column experiments," Journal of the Structural Division, Vol. 90, No. ST2, pp. 223-256.

van Manen, S.E., 1982, "Plastic Design of Braced Frames Allowing Plastic Hinges in the Columns," Heron, Vol. 27, No. 2, pp. 35-45.

Varma, A.H., Ricles, J.M., Sause, R., and Lu, L.W., 2002, "Seismic behavior and modeling of high-strength composite concrete-filled steel tube (CFT) beamcolumns," Journal of Constructional Steel Research, Vol. 58, Nos. 5-8, pp. 725-758.

Varma, A.H., Ricles, J.M., Sause, R., and Lu, L.W., 2004, "Seismic behavior and design of high-strength square concrete-filled steel tube beam-columns," Journal of Structural Engineering, Vol. 130, No. 2, pp. 169-179.

Venti, M. and Engelhardt, M.D., 2000, Test of a Free Flange Connection with a Composite Floor Slab, SAC Steel Project, Report No. SAC 00/18, prepared by the SAC Joint Venture, a partnership of the Structural Engineers Association of California (SEAOC), Applied Technology Council (ATC), and California Universities for Research in Earthquake Engineering (CUREe).

Vinnakota, S., 1977, Finite Difference Method for Plastic Beam-Columns, " in Theory of Beam-Columns, eds. W. F. Chen and T. Atsuta, McGraw-Hill, New York, New York.

Vinnakota, S., 1982, "Planar strength of restrained beam-columns," Journal of the Structural Division, Vol. 108, No. ST11, pp. 2496-2516.

Vinnakota, S., 1983, "Planar strength of directionally and rotationally restrained steel columns," Proceedings, $3^{\text {rd }}$ Int. Colloq. Stab. Met. Struct., Toronto, Canada. 
Vinnakota, S., 1984, “Closure,” Journal of the Structural Division, Vol. 110, No. ST2, pp. 43-435.

Wald, F., Bouguin, V., Sokol, Z., and Muzeau, J., 2000, "Effective length of t-stub of rhs column base plates," Proceedings, Connections in Steel Structures IV, Roanoke, Virginia.

Wald, F., Sokol, Z., Šimek, I., Bazantová, Z., 1995, “Column base semi-rigid behavior," Poddajnost patek ocelových skeletu. (in Czech), Rep. G 8118, Czech Technical University, Praha, p. 52.

Wang, S.J., 1988, Seismic Response of Steel Building Frames with Inelastic Joint Deformation, Ph.D. Dissertation, Department of Civil Engineering, Lehigh University, Bethlehem, Pennsylvania.

Wang, T., Mosqueda, G., Jacobsen, A., and Delgado, M.C., 2011, "Performance evaluation of a distributed hybrid test framework to reproduce the collapse behaviour of a structure," Earthquake Engineering and Structural Dynamics, DOI: $10.1002 /$ eqe. 1130 .

Wen, Y.K., 1980, "Equivalent linearization for hysteretic systems under random excitation," Journal of Applied Mechanics, Vol. 47, pp. 150-154.

Whittaker, A.S. and Gilani, A.S.J., 1996, Cyclic Testing of Steel Beam-Column Connections, Rep. EERC-STI 1996/04, Earthquake Engineering Research Center, University of California, Berkeley, California.

Yamanaka, K. and Yamada, S., 1984, "Elasto-plastic deformation and fracture behavior of steel box columns subjected to double curvature cyclic bending under constant axial load," Journal of Structural and Construction Engineering.

Young, B.W., 1973, "The in-plane failure of steel beam-columns," The Structural Engineer, Vol. 51, No. 1, pp. 27-35.

Yu, C. K. and Tall, L., 1971, “A514 Steel beam-columns,” Publ. IABSE, No. 31-II, pp. 185-213.

Yu, Q.S., Gilton, C., and Uang, C.M., 2000, Cyclic Response of RBS Moment Connections: Loading Sequence and Lateral Bracing Effects, SAC Steel Project Report 00/22, prepared by the SAC Joint Venture, a partnership of the Structural Engineers Association of California (SEAOC), Applied Technology Council (ATC), and California Universities for Research in Earthquake Engineering (CUREe).

Zareian, F. and Krawinkler, H., 2007, "Assessment of probability of collapse and design for collapse safety," Earthquake Engineering and Structural Dynamics, Vol. 36, No. 13, pp. 1901-1914. 
Zareian, F., Lignos, D.G., and Krawinkler, H., 2011, "Seismic design modification factors for steel SMRFs for uniform collapse safety," Protection of the Built Environment Against Earthquakes, Springer, New York, New York.

Zeimian, R.D., 2010, Guide to Stability Design Criteria for Metal Structures, $6^{\text {th }}$ Edition, Structural Stability Research Council and John Wiley \& Sons, Inc., 1078 pages, New York, New York.

Zheng, Y., Usami, T., and Ge, H., 2000, "Ductility of thin-walled steel box stubcolumns," Journal of Structural Engineering, Vol. 126, Vol. 11, pp. 13041311. 



\section{Project Participants}

\author{
National Institute of Standards and Technology \\ John (Jack) R. Hayes, Jr. \\ Engineering Laboratory (MS8604) \\ National Institute of Standards and Technology \\ 100 Bureau Drive \\ Gaithersburg, Maryland 20899 \\ www.NEHRP.gov \\ John (Jay) L. Harris III \\ Engineering Laboratory (MS8603) \\ National Institute of Standards and Technology \\ 100 Bureau Drive \\ Gaithersburg, Maryland 20899 \\ www.NEHRP.gov

\section{NEHRP Consultants Joint Venture} \\ APPLIED TECHNOLOGY COUNCIL \\ 201 Redwood Shores Parkway, Suite 240 \\ Redwood City, California 94065 \\ www.ATCouncil.org
}

\section{Joint Venture Management Committee}

James R. Harris

J.R. Harris \& Company

1775 Sherman Street, Suite 1525

Denver, Colorado 80203

Robert Reitherman

Consortium of Universities for Research in

Earthquake Engineering

1301 S. $46^{\text {th }}$ Street, Building 420

Richmond, California 94804

\section{Joint Venture Program Committee}

Jon A. Heintz (Program Manager)

Applied Technology Council

201 Redwood Shores Parkway, Suite 240

Redwood City, California 94065
Steven L. McCabe

Engineering Laboratory (MS8604)

National Institute of Standards and Technology

100 Bureau Drive

Gaithersburg, Maryland 20899

www.NEHRP.gov
CONSORTIUM OF UNIVERSITIES FOR

RESEARCH IN EARTHQUAKE ENGINEERING

1301 S. $46^{\text {th }}$ Street, Building 420

Richmond, California 94804

www.CUREE.org

Christopher Rojahn

Applied Technology Council

201 Redwood Shores Parkway, Suite 240

Redwood City, California, 94065

Andrew Whittaker

University at Buffalo

Dept. of Civil, Structural, and Environ. Engin.

230 Ketter Hall

Buffalo, New York 14260

Michael Constantinou

University at Buffalo

Dept. of Civil, Structural, and Environ. Engin.

132 Ketter Hall

Buffalo, New York 14260 
C.B. Crouse

URS Corporation

$15014^{\text {th }}$ Avenue, Suite 1400

Seattle, Washington 98101

William T. Holmes

Rutherford \& Chekene

55 Second Street, Suite 600

San Francisco, California 94105

\section{Project Manager}

Ayse Hortacsu

Applied Technology Council

201 Redwood Shores Parkway, Suite 240

Redwood City, California, 94065

\section{Project Technical Committee}

James O. Malley (Technical Director)

Degenkolb Engineers

235 Montgomery Street, Suite 500

San Francisco, California 94104

Charles J. Carter

American Institute of Steel Construction

1 East Wacker Drive, Suite 700

Chicago, Illinois 60601

Jerome F. Hajjar

Northeastern University

Dept. of Civil and Environmental Engineering

400 Snell Engineering Center

360 Huntington Avenue

Boston, Massachusetts 02115

\section{FEMA Representative}

Helmut Krawinkler (FEMA Technical Monitor)

Stanford University

Dept. of Civil and Environmental Engineering

473 Via Ortega

Stanford, California 94305
Jack Moehle

University of California, Berkeley

Dept. of Civil and Environmental Engineering

325 Davis Hall - MC 1792

Berkeley, California 94720

James R. Harris (ex-officio)

Andrew Whittaker (ex-officio)
Dimitrios Lignos

McGill University

Dept. of Civil Engineering and Applied Mechanics

817 Sherbrooke West, Room 488

Montreal, Quebec, H3A 2K6

Charles Roeder

University of Washington

Dept. of Civil and Environmental Engineering

233-B More Hall, Box 2700

Seattle, Washington 98195

Mark Saunders

Rutherford \& Chekene

55 Second Street, Suite 600

San Francisco, California 94105 